UNIVERSIDADE DE SÃO PAULO

ESCOLA DE ARTES, CIÊNCIAS E HUMANIDADES

PROGRAMA DE PÓS-GRADUAÇÃO EM SISTEMAS DE INFORMAÇÃO

JOÃO CARLOS SILVA DE SOUZA

Aprendizado semi-supervisionado para o tratamento de incerteza na rotulação de dados de Química Medicinal

São Paulo 
JOÃO CARLOS SILVA DE SOUZA

\title{
Aprendizado semi-supervisionado para o tratamento de incerteza na rotulação de dados de Química Medicinal
}

\author{
Versão Corrigida
}

Dissertação apresentada à Escola de Artes, Ciências e Humanidades da Universidade de São Paulo para obtenção do título de Mestre em Ciências pelo Programa de Pós-graduação em Sistemas de Informação.

Área de concentração: Sistemas de Informação

Versão corrigida contendo as alterações solicitadas pela comissão julgadora em 09 de março de 2017. A versão original encontra-se em acervo reservado na Biblioteca da EACH-USP e na Biblioteca Digital de Teses e Dissertações da USP (BDTD), de acordo com a Resolução CoPGr 6018, de 13 de outubro de 2011.

Orientador: Profa. Dra. Patrícia Rufino Oliveira

São Paulo 
Autorizo a reprodução e divulgação total ou parcial deste trabalho, por qualquer meio convencional ou eletrônico, para fins de estudo e pesquisa, desde que citada a fonte.

CATALOGAÇÃO-NA-PUBLICAÇÃO

(Universidade de São Paulo. Escola de Artes, Ciências e Humanidades. Biblioteca)

Souza, João Carlos Silva de

Aprendizado semi-supervisionado para o tratamento de incerteza na rotulação de dados de química medicinal / João Carlos Silva de Souza ; orientadora, Patrícia Rufino Oliveira. - São Paulo, 2017.

115 p. : il

Dissertação (Mestrado em Ciências) - Programa de Pós-

Graduação em Sistemas de Informação, Escola de Artes, Ciências e Humanidades, Universidade de São Paulo.

Versão corrigida

1. Química farmacêutica. 2. Tratamento de incerteza. 3.

Aprendizado semi-supervisionado. 4. Máquinas de aprendizado extremo. 5. Aprendizado computacional. 6. Bioinformática. I.

Oliveira, Patrícia Rufino, orient. II. Título

CDD 22.ed.- 615.19 
Dissertação de autoria de João Carlos Silva de Souza, sob o título "Aprendizado semi-supervisionado para o tratamento de incerteza na rotulação de dados de Química Medicinal", apresentada à Escola de Artes, Ciências e Humanidades da Universidade de São Paulo, para obtenção do título de Mestre em Ciências pelo Programa de Pós-graduação em Sistemas de Informação, na área de concentração Metodologia e Técnicas da Computação, aprovada em 09 de março de 2017 pela comissão julgadora constituída pelos doutores:

Profa. Dra. Patrícia Rufino Oliveira

Universidade Federal de São Paulo

Presidente

Prof. Dr. Clodoaldo Aparecido de Moraes Lima

Universidade de São Paulo

Prof. Dr. Jesus Pascual Mena Chalco

Universidade Federal do ABC

Prof. Dr. Gustavo Enrique de Almeida Prado Alves

Universidade de São Paulo 


\section{Agradecimentos}

Agradeço primeiramente a Deus, por me auxiliar nesta caminhada, me permitindo aprender uma nova lição a cada momento de desespero e me fortalecendo e encorajando a sempre dar mais um passo na direção de meus objetivos.

Agradeço também a minha orientadora Profa. Dra. Patrícia Rufino Oliveira e a Profa. Dra Kathia Maria Honório por acreditarem em meu potencial e me ajudarem a atingir o meu melhor.

Agradeço ao meu grupo de pesquisa, em especial a Rodolfo,Suzana e Weslane por me ouvirem nos momentos difíceis e não me deixarem desistir. A todos os professores do PpgSi, que me doaram um pouco do que sabem, contribuição essa gigantesca para a execução deste trabalho.

A meus pais Maria da Conceição Silva de Souza e Jesus Mariano de Souza (em memória) que mesmo com pouca instrução, foram capazes de me mostrar o quão libertador o conhecimento pode ser. A minha esposa Sávia Xavier de Souza pelo incentivo e compreensão e a minha pequena filha Agatha Xavier de Souza, por me inspirar a cada segundo de minha vida. 
Os segredos da alma humana podem ser comparados a imensidão do mar, mas o pó de seus restos mortais não enchem a palma de suas mãos.

(Autor desconhecido) 


\section{Resumo}

\section{SOUZA, João Carlos Silva de. Aprendizado semi-supervisionado para o tratamento de incerteza na rotulação de dados de Química Medicinal. 2017. 115 f. Dissertação (Mestrado em Ciências) - Escola de Artes, Ciências e Humanidades, Universidade de São Paulo, São Paulo, 2017. Versão Corrigida.}

Nos últimos 30 anos, a área de aprendizagem de máquina desenvolveu-se de forma comparável com a Física no início do século XX. Esse avanço tornou possível a resolução de problemas do mundo real que anteriormente não poderiam ser solucionados por máquinas, devido à dificuldade de modelos puramente estatísticos ajustarem-se de forma satisfatória aos dados de treinamento. Dentre tais avanços, pode-se citar a utilização de técnicas de aprendizagem de máquina na área de Química Medicinal, envolvendo métodos de análise, representação e predição de informação molecular por meio de recursos computacionais. Os dados utilizados no contexto biológico possuem algumas características particulares que podem influenciar no resultado de sua análise. Dentre estas, pode-se citar a complexidade das informações moleculares, o desbalanceamento das classes envolvidas e a existência de dados incompletos ou rotulados de forma incerta. Tais adversidades podem prejudicar o processo de identificação de compostos candidatos a novos fármacos, se não forem tratadas de forma adequada. Neste trabalho, foi abordada uma técnica de aprendizagem de máquina semi-supervisionada capaz de reduzir o impacto causado pelo problema da incerteza na rotulação dos dados, aplicando um método para estimar rótulos mais confiáveis para os compostos químicos existentes no conjunto de treinamento. Na tentativa de evitar os efeitos causados pelo desbalanceamento dos dados, foi incorporada ao processo de estimação de rótulos uma abordagem sensível ao custo, com o objetivo de evitar o viés em benefício da classe majoritária. Após o tratamento do problema da incerteza na rotulação, classificadores baseados em Máquinas de Aprendizado Extremo foram construídos, almejando boa capacidade de aproximação em um tempo de processamento reduzido em relação a outras abordagens de classificação comumente aplicadas. Por fim, o desempenho dos classificadores construídos foi avaliado por meio de análises dos resultados obtidos, confrontando o cenário com os dados originais e outros com as novas rotulações obtidas durante o processo de estimação semi-supervisionado.

Palavras-chave: Química Farmacêutica, Tratamento de Incerteza, Aprendizado semisupervisionado, Maximização da Esperança, Máquinas de Aprendizado Extremo. 


\begin{abstract}
SOUZA, João Carlos Silva de. Semi supervised learning for uncertainty on medicinal chemistry labelling. 2017. 115 p. Dissertation (Master of Science) - School of Arts, Sciences and Humanities, University of São Paulo, São Paulo, 2017. Corrected Version.

In the last 30 years, the area of machine learning has developed in a way comparable to Physics in the early twentieth century. This breakthrough has made it possible to solve realworld problems that previously could not be solved by machines because of the difficulty of purely statistical models to fit satisfactorily with training data. Among these advances, one can cite the use of machine learning techniques in the area of Medicinal Chemistry, involving methods for analysing, representing and predicting molecular information through computational resources. The data used in the biological context have some particular characteristics that can influence the result of its analysis. These include the complexity of molecular information, the imbalance of the classes involved, and the existence of incomplete or uncertainly labeled data. If they are not properly treated, such adversities may affect the process of identifying candidate compounds for new drugs. In this work, a semi-supervised machine learning technique was considered to reduce the impact caused by the problem of uncertainty in the data labeling, by applying a method to estimate more reliable labels for the chemical compounds in the training set. In an attempt to reduce the effects caused by data imbalance, a cost-sensitive approach was incorporated to the label estimation process, in order to avoid bias in favor of the majority class. After addressing the uncertainty problem in labeling, classifiers based on Extreme Learning Machines were constructed, aiming for good approximation ability in a reduced processing time in relation to other commonly applied classification approaches. Finally, the performance of the classifiers constructed was evaluated by analyzing the results obtained, comparing the scenario with the original data and others with the new labeling obtained by the semi-supervised estimation process.
\end{abstract}

Keywords: Medicinal Chemistry, Uncertainty handling, semi-supervised learning, Expectation and Maximization, Extreme Learning Machines. 


\section{Lista de figuras}

Figura 1 - Subdivisão de conjunto no Aprendizado PU - O conjunto de treinamento $\boldsymbol{X}$ possui todos os elementos $x_{i}$ rotulados e não rotulados. Em seguida, o conjunto dos elementos positivos, aqui identificados como $\boldsymbol{P}$, é separado dos elementos do conjunto $\boldsymbol{U}$ que corresponde ao conjunto não rotulado. Uma parte do conjunto $\boldsymbol{P}$ denominada de espiões $\boldsymbol{S}$, é agrupada ao conjunto $\boldsymbol{U}$. Em seguida, o algoritmo I-EM se encarrega de fornecer uma nova rotulação aos elementos, identificando as instâncias com rotulação confiavelmente negativa incluídas em um novo conjunto identificado por

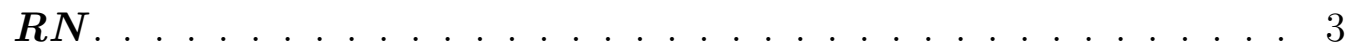

Figura 2 - Arquitetura de uma rede SLFN - Demonstração da estrutura de uma rede SLFN contendo uma camada de entrada com m nós, uma única camada oculta com n nós e uma camada de saída com m nós. .... . 43

Figura 3 - Classificadores discretos (A,B,C,D e E) posicionados no espaço ROC. Para os classificadores localizados no triângulo superior esquerdo, quanto mais afastados os pontos da linha pontilhada, melhor o desempenho. Os classificadores localizados abaixo da linha pontilhada possuem desempenho menor que 50\%, considerando uma escolha aleatória de classe. 52

Figura 4 - Fluxo de tarefas do modelo prposto, que envolve tratamento dos dados, estimação de rótulos e classificação dos dados. . . . . . . . . . . . . . . . 54

Figura 5 - (a) Projeção via PCA conjunto de dados Iris com os elementos da classe setosa definidas como positivas e os demais elementos versicolor e virgínica definidos como negativos. (b) Projeção via PCA do conjunto de dados Iris após modificação de 30\% dos rótulos positivos. . . . . . . 58

Figura 6 - (a) Rotulação inicial contendo as intâncias positivas com $30 \%$ dos elementos alterados e 10\% dos objetos positivos utilizados como espiões.

(b) Rotulação estimada pelo método PU. . . . . . . . . . . . . . . . . . 59

Figura 7 - Análise ROC para classificadores com fator de custo $C_{i j}=\frac{1}{\text { total }_{p}+\text { total }_{n}}$ para o conjunto de dados AID1284. (a) Para dados com rotulação original (b) Para dados com rotulação após PU. . . . . . . . . . . . . . 67 
Figura 8 - Análise ROC para classificadores com fator de custo $C_{i j}=\frac{0.5}{\text { total }_{p}+\text { total }_{n}}$ para o conjunto de dados AID1284. (a) Para dados com rotulação original (b) Para dados com rotulação após PU. . . . . . . . . . . . . .

Figura 9 - Análise ROC para classificadores com fator de custo $C_{i j}=1.0$ para o conjunto de dados AID1284. (a) Para dados com rotulação original (b) Para dados com rotulação após PU. . . . . . . . . . . . . . .

Figura 10 - Análise ROC para classificadores com fator de custo $C_{i j}=1.0+\frac{\text { total }_{p}}{\text { total }_{n}}$ para conjunto de dados AID1284. (a) Para dados com rotulação original (b) Para dados com rotulação após PU. . . . . . . . . . . . . . . . .

Figura 11 - Análise ROC para classificadores com fator de custo $C_{i j}=\frac{1}{\text { total }_{p}+\text { total }_{n}}$ para conjunto de dados AID721. (a) Para dados com rotulação original (b) Para dados com rotulação após PU. . . . . . . . . . . . . . . . . 76

Figura 12 - Análise ROC para classificadores com fator de custo $C_{i j}=0.5+$ $\frac{1}{\text { total }_{p}+\text { total }_{n}}$ para o conjunto de dados AID721. (a) Para dados com rotulação original (b) Para dados com rotulação após PU.

Figura 13 - Análise ROC para classificadores com fator de custo $C_{i j}=1.0$ para conjunto de dados 721. (a) Para dados com rotulação original (b) Para dados com rotulação após PU. . . . . . . . . . . . . . . . .

Figura 14 - (a)Modelos produzidos utilizando $C_{i j}=1.0+\frac{\text { total }_{p}}{\text { total }_{n}}$ para conjunto de dados AID721. (b)Modelos utilizando $C_{i j}=1.0+\frac{\text { total }_{p}}{\text { total }_{n}}$ para conjunto de dados AID721, com PU. . . . . . . . . . . . . . . . . . 81

Figura 15 - (a)Modelos produzidos utilizando $C_{i j}=\frac{1}{\text { total }_{p}+\text { total }_{n}}$ para o conjunto de dados AID644. (b)Modelos utilizando $C_{i j}=\frac{1}{\text { total }_{p}+\text { total }_{n}}$ para o conjunto de dados AID644, com PU. . . . . . . . . . . . . . . . . . . . 84

Figura 16 - (a) Modelos produzidos utilizando $C_{i j}=0.5+\frac{\text { total }_{p}}{\text { total }_{n}}$ para o conjunto de dados AID644. (b)Modelos utilizando $C_{i j}=0.5+\frac{\text { total }_{p}}{\text { total }_{n}}$ para o conjunto de dados AID644, com PU. . . . . . . . . . . . . . 85

Figura 17 - (a)Modelos produzidos utilizando $C_{i j}=1.0$ para o conjunto de dados AID644. (b)Modelos utilizando $C_{i j}=1.0$ para o conjunto de dados AID644, com PU. . . . . . . . . . . . . . . . . .

Figura 18 - (a)Modelos produzidos utilizando $C_{i j}=1.0+\frac{\text { total }_{p}}{\text { total }_{n}}$ para o conjunto de dados AID644. (b)Modelos utilizando $C_{i j}=1.0+\frac{\text { total }_{p}}{\text { total }_{n}}$ para o conjunto de dados AID644, com PU. . . . . . . . . . . . . . . 89 
Figura 19 - (a)Modelos produzidos utilizando $C_{i j}=\frac{1}{\text { total }_{p}+\text { total }_{n}}$ para o conjunto de dados AID439. (b)Modelos utilizando $C_{i j}=\frac{1}{\text { total }_{p}+\text { total }_{n}}$ para o conjunto de dados AID439, aplicando PU. . . . . . . . . . . . . . . . . 92

Figura 20 - (a)Modelos produzidos utilizando $C_{i j}=0.5+\frac{\text { total }_{p}}{\text { total }_{n}}$ para o conjunto de dados AID439. (b)Modelos utilizando $C_{i j}=0.5+\frac{\text { total }_{p}}{\text { total }_{n}}$ para o conjunto de dados AID439, com PU. . . . . . . . . . . . . . . . 94

Figura 21 - (a)Modelos produzidos utilizando $C_{i j}=1.0$ para o conjunto de dados AID439. (b)Modelos utilizando $C_{i j}=1.0$ para o conjunto de dados AID439, com PU. . . . . . . . . . . . . . . . . 95

Figura 22 - (a)Modelos produzidos utilizando $C_{i j}=1.0+\frac{\text { total }_{p}}{\text { total }_{n}}$ para o conjunto de dados AID439. (b)Modelos utilizando $C_{i j}=1.0+\frac{\text { total }_{p}}{\text { total }_{n}}$ para o conjunto de dados AID439, com PU. . . . . . . . . . . . . . . . . . 97

Figura 23 - (a)Modelos utilizando $C_{i j}=\frac{1}{\text { total }_{p}+\text { total }_{n}}$ para o conjunto de dados AID1608.(b)Modelos utilizando $C_{i j}=\frac{1}{\text { total }_{p}+\text { total }_{n}}$ para o conjunto de dados AID1608, com PU. . . . . . . . . . . . . . . . 100

Figura 24 - (a)Modelos utilizando $C_{i j}=0.5+\frac{\text { total }_{p}}{\text { total }_{n}}$ para o conjunto de dados AID1608. (b)Modelos utilizando $C_{i j}=0.5+\frac{\text { total }_{p}}{\text { total }_{n}}$ para o conjunto de dados AID1608, com PU. . . . . . . . . . . . . . 101

Figura 25 - (a)Modelos utilizando $C_{i j}=1.0$ para o conjunto de dados AID1608. (b)Modelos utilizando $C_{i j}=1.0$ para o conjunto de dados AID1608, com PU. . . . . . . . . . . . . . . . . . . . . . 103

Figura 26 - (a)Modelos utilizando $C_{i j}=1.0+\frac{\text { total }_{p}}{\text { total }_{n}}$ para o conjunto de dados AID1608. (b)Modelos utilizando $C_{i j}=1.0+\frac{\text { total }_{p}}{\text { total }_{n}}$ para o conjunto de dados AID1608, com PU. . . . . . . . . . . . . . . 105 


\section{Lista de algoritmos}

Algoritmo 1 - Aprendizado PU Adaptado . . . . . . . . . . . . . . . . . . 41 


\section{Lista de tabelas}

Tabela 1 - Matriz de Custos . . . . . . . . . . . . . . 36

Tabela 2 - Matriz de Confusão . . . . . . . . . . . . . . . . . . . 49

Tabela 3 - Distribuição de classes do conjunto Iris antes e após alteração da rotulação. 56

Tabela 4 - Distribuições de classes obtidas após aplicação do algoritmo PU ao conjunto de dados Iris com 30\% dos exemplos da classe positiva modificados para classe negativa. Contempla-se, ainda, a aplicação de técnicas de discretização como Equal Width (EW) e Equal Frequency (EF) e padronização na fase de pré-processamento dos dados. . . . . . . . . . 57

Tabela 5 - Melhores resultados obtidos pelo classificador ELM para os dados originais, considerando variações do número de neurônios e funções de ativação. Resultados ordenados pela métrica f-score. . . . . . . . . . . 60

Tabela 6 - Melhores resultados obtidos pelo classificador ELM para os dados com rotulação alterada, considerando variações do número de neurônios e funções de ativação. Resultados ordenados pela métrica f-score. . . . .

Tabela 7 - Melhores resultados obtidos pelo classificador ELM para os dados com rotulação alterada, considerando variações do número de neurônios e funções de ativação. Resultados ordenados pela métrica f-score. . . . . 61

Tabela 8 - Informações resumidas sobre os conjuntos de dados de QM analisados. 64 Tabela 9 - Distribuições de classes do conjunto de dados AID1284 obtidas após a execução do processo PU, com variação de fatores de custo. . . . . . . .

Tabela 10 - Resultados obtidos pelo classificador ELM com fator de custo $C_{i j}=$ $\frac{1}{\text { total }_{p}+\text { total }_{n}}$ para o conjunto de dados AID1284 com rotulação original. . 66

Tabela 11 - Resultados obtidos pelo classificador ELM com fator de custo $C_{i j}=$ $\frac{1}{\text { total }_{p}+\text { total }_{n}}$ para o conjunto de dados AID1284 após a aplicação do rotulador PU. . . . . . . . . . . . . . . . . . 66

Tabela 12 - Resultados obtidos pelo classificador ELM com fator de custo $C_{i j}=$ $\frac{0.5}{\text { total }_{p}+\text { total }_{n}}$ para o conjunto de dados AID1284 com rotulação original. .

Tabela 13 - Resultados obtidos pelo classificador ELM com fator de custo $C_{i j}=$ $\frac{0.5}{\text { total }_{p}+\text { total }_{n}}$ para o conjunto de dados AID1284 após a aplicação do rotulador PU. . . . . . . . . . . . . . . . . . . . 
Tabela 14 - Resultados obtidos pelo classificador ELM com fator de custo 1.0 para o conjunto de dados AID1284 antes da aplicação do rotulador PU. . 70

Tabela 15 - Resultados obtidos pelo classificador ELM com fator de custo 1.0 para o conjunto de dados AID1284 após a aplicação do rotulador PU. . . . . 70

Tabela 16 - Resultados obtidos pelo classificador ELM com fator de custo $C_{i j}=1.0$ $+\frac{1}{\text { total }_{p}+\text { total }_{n}}$ para o conjunto de dados AID1284 antes da aplicação do rotulador PU. . . . . . . . . . . . . . . . . . 72

Tabela 17 - Resultados obtidos pelo classificador ELM com fator de custo $C_{i j}=1.0$ $+\frac{1}{\text { total }_{p}+\text { total }_{n}}$ para o conjunto de dados AID1284 após a aplicação do rotulador PU. . . . . . . . . . . . . . . . 7

Tabela 18 - Distribuições de classes obtidas após a execução do processo PU contemplando a variação dos custos para o conjunto de dados AID721. . .

Tabela 19 - Resultados obtidos pelo classificador ELM com fator de custo $C_{i j}=$ $\frac{1}{\text { total }_{p}+\text { total }_{n}}$ para o conjunto de dados AID721 antes da aplicação do rotulador PU.

Tabela 20 - Resultados obtidos pelo classificador ELM com fator de custo $C_{i j}$ $=\frac{1}{\text { total }_{p}+\text { total }_{n}}$ para o conjunto de dados AID721 após a aplicação do rotulador PU. . . . . . . . . . . . . . . . . . . 75

Tabela 21 - Resultados obtidos pelo classificador ELM comfator de custo $C_{i j}=0.5$ $+\frac{1}{\text { total }_{p}+\text { total }_{n}}$ para o conjunto de dados AID721 antes da aplicação do rotulador PU. . . . . . . . . . . . . . . . . 76

Tabela 22 - Resultados obtidos pelo classificador ELM com fator de custo $C_{i j}=$ $0.5+\frac{1}{\text { total }_{p}+\text { total }_{n}}$ para o conjunto de dados AID721 após a aplicação do rotulador PU. . . . . . . . . . . . . . . . . 77

Tabela 23 - Resultados obtidos pelo classificador ELM com fator de custo 1.0 para o conjunto de dados AID721 antes da aplicação do rotulador PU. . . .

Tabela 24 - Resultados obtidos pelo classificador ELM com fator de custo 1.0 para o conjunto de dados AID721 após a aplicação do rotulador PU. ... . 79

Tabela 25 - Resultados obtidos pelo classificador ELM com fator de custo $C_{i j}=1.0$ $+\frac{1}{\text { total }_{p}+\text { total }_{n}}$ para o conjunto de dados AID721 antes da aplicação do rotulador PU. 
Tabela 26 - Resultados obtidos pelo classificador ELM com risco $C_{i j}=1.0+$ $\frac{1}{\text { total }_{p}+\text { total }_{n}}$ para o conjunto de dados AID721 após a aplicação do rotulador PU. . . . . . . . . . . . . . . . . . . 80

Tabela 27 - Distribuições de classes obtidas após a execução do processo PU contemplando a variação dos custos para o conjunto de dados AID644. . . 82

Tabela 28 - Resultados obtidos pelo classificador ELM com fator de custo $C_{i j}=$ $\frac{1}{\text { total }_{p}+\text { total }_{n}}$ para o conjunto de dados AID644 antes da aplicação do rotulador PU. . . . . . . . . . . . . . . . . . . 83

Tabela 29 - Resultados obtidos pelo classificador ELM com risco $C_{i j}=\frac{1}{\text { total }_{p}+\text { total }_{n}}$ para o conjunto de dados AID644 após a aplicação do rotulador PU. 83

Tabela 30 - Resultados obtidos pelo classificador ELM com risco $C_{i j}=0.5+$ $\frac{1}{\text { total }_{p}+\text { total }_{n}}$ para o conjunto de dados AID644 antes da aplicação do rotulador PU. . . . . . . . . . . . . . . . . . .

Tabela 31 - Resultados obtidos pelo classificador ELM com risco $C_{i j}=0.5+$ $\frac{1}{\text { total }_{p}+\text { total }_{n}}$ para o conjunto de dados AID644 após a aplicação do rotulador PU. . . . . . . . . . . . . . . . . . . .

Tabela 32 - Resultados obtidos pelo classificador ELM com risco 1.0 para o conjunto de dados AID644 antes da aplicação do rotulador PU. . . . . . . . . . . 86

Tabela 33 - Resultados obtidos pelo classificador ELM com risco 1.0 para o conjunto de dados AID644 após a aplicação do rotulador PU. . . . . . . . . . . 86

Tabela 34 - Resultados obtidos pelo classificador ELM com risco $C_{i j}=1.0+$ $\frac{1}{\text { total }_{p}+\text { total }_{n}}$ para o conjunto de dados AID644 antes da aplicação do rotulador PU. . . . . . . . . . . . . . . . .

Tabela 35 - Resultados obtidos pelo classificador ELM com risco $C_{i j}=1.0+$ $\frac{1}{\text { total }_{p}+\text { total }_{n}}$ para o conjunto de dados AID644 após a aplicação do rotulador PU. . . . . . . . . . . . . . . . . . . . .

Tabela 36 - Distribuições de classes obtidas após a execução do processo PU contemplando a variação dos custos para o conjunto de dados AID439. . . 90

Tabela 37 - Resultados obtidos pelo classificador ELM com risco $C_{i j}=\frac{1}{\text { total }_{p}+\text { total }_{n}}$ para o conjunto de dados AID439 antes da aplicação do rotulador PU.

Tabela 38 - Resultados obtidos pelo classificador ELM com risco $C_{i j}=\frac{1}{\text { total }_{p}+\text { total }_{n}}$ para o conjunto de dados AID439 após a aplicação do rotulador PU. 
Tabela 39 - Resultados obtidos pelo classificador ELM com risco $C_{i j}=0.5+$ $\frac{1}{\text { total }_{p}+\text { total }_{n}}$ para o conjunto de dados AID439 antes da aplicação do rotulador PU. . . . . . . . . . . . . . . . . . . 92

Tabela 40 - Resultados obtidos pelo classificador ELM com risco $C_{i j}=0.5+$ $\frac{1}{\text { total }_{p}+\text { total }_{n}}$ para o conjunto de dados AID439 após a aplicação do rotulador PU. . . . . . . . . . . . . . . . . . . . . 93

Tabela 41 - Resultados obtidos pelo classificador ELM com risco 1.0 para o conjunto de dados AID439 antes da aplicação do rotulador PU. . . . . . . . . . . 94

Tabela 42 - Resultados obtidos pelo classificador ELM com risco 1.0 para o conjunto de dados AID439 após a aplicação do rotulador PU. . . . . . . . . . . 95

Tabela 43 - Resultados obtidos pelo classificador ELM com risco $C_{i j}=1.0+$ $\frac{1}{\text { total }_{p}+\text { total }_{n}}$ para o conjunto de dados AID439 antes da aplicação do rotulador PU. . . . . . . . . . . . . . . . . . . . . 96

Tabela 44 - Resultados obtidos pelo classificador ELM com risco $C_{i j}=1.0+$ $\frac{1}{\text { total }_{p}+\text { total }_{n}}$ para o conjunto de dados AID439 após a aplicação do rotulador PU. . . . . . . . . . . . . . . . . . . . . 96

Tabela 45 - Distribuições de classes obtidas após a execução do processo PU contemplando a variação dos custos para o conjunto de dados AID1608.

Tabela 46 - Resultados obtidos pelo classificador ELM com risco $C_{i j}=\frac{1}{\text { total }_{p}+\text { total }_{n}}$ para o conjunto de dados AID1608 antes da aplicação do rotulador PU. 99

Tabela 47 - Resultados obtidos pelo classificador ELM com risco $C_{i j}=\frac{1}{\text { total }_{p}+\text { total }_{n}}$ para o conjunto de dados AID1608 após a aplicação do rotulador PU. .

Tabela 48 - Resultados obtidos pelo classificador ELM com risco $C_{i j}=0.5+$ $\frac{1}{\text { total }_{p}+\text { total }_{n}}$ para o conjunto de dados AID1608 antes da aplicação do rotulador PU.

Tabela 49 - Resultados obtidos pelo classificador ELM com risco $C_{i j}=0.5+$ $\frac{1}{\text { total }_{p}+\text { total }_{n}}$ para o conjunto de dados AID1608 após a aplicação do rotulador PU. . . . . . . . . . . . . . . . . . . . . . 101

Tabela 50 - Resultados obtidos pelo classificador ELM com risco 1.0 para o conjunto de dados AID1608 antes da aplicação do rotulador PU. . . . . . . . . . 102

Tabela 51 - Resultados obtidos pelo classificador ELM com risco 1.0 para o conjunto de dados AID1608 após a aplicação do rotulador PU. . . . . . . . . . . 102 
Tabela 52 - Resultados obtidos pelo classificador ELM com risco $C_{i j}=1.0+$ $\frac{1}{\text { total }_{p}+\text { total }_{n}}$ para o conjunto de dados AID1608 antes da aplicação do rotulador PU. . . . . . . . . . . . . . . . . . . . 103

Tabela 53 - Resultados obtidos pelo classificador ELM com risco $C_{i j}=1.0+$ $\frac{1}{\text { total }_{p}+\text { total }_{n}}$ para o conjunto de dados AID1608 após a aplicação do rotulador PU. . . . . . . . . . . . . . . . . . . . . . . . . 104

Tabela 54 - Resultados de classificação obtidos neste trabalho em comparação com outros obtidos em trabalhos anteriores. . . . . . . . . . . . . 106

Tabela 55 - Tabela contendo os compostos que apresentaram alteração na rotulação em diversas configurações de peso, presentes no conjunto AID439. . . . 107

Tabela 56 - Resultados obtidos após a aplicação do teste $t$ e valores críticos para análise bicaudal de $95 \%$. . . . . . . . . . . . . . . . 108 


\section{Lista de símbolos}

\begin{tabular}{|c|c|}
\hline$v$ & Hipótese provável \\
\hline$V$ & Conjunto de hipóteses prováveis \\
\hline$D$ & Conjunto de dados de Treinamento \\
\hline$v_{\mathrm{NB}}$ & Classificador Naive Bayes \\
\hline$n$ & Número total de elementos \\
\hline$a$ & Atributo de uma instância \\
\hline$<a_{1}, a_{2}, \ldots, a_{n}$ & ${ }_{n}>$ Vetor de características de uma determinada instância \\
\hline$d$ & Uma instância formada pelo vetor de características $\left[<a_{1}, a_{2}, \ldots, a_{n}>\right.$ \\
\hline$\delta$ & Probabilidade condicional \\
\hline$\theta$ & Parâmetros de configuração do classificador Naive Bayes \\
\hline$T$ & Número de iterações totais definidas pelo usuário \\
\hline$t$ & Iteração atual do classificador \\
\hline$\sigma$ & Função de ativação \\
\hline$x$ & Valor de entrada da Rede Neural \\
\hline$w$ & Vetor de pesos \\
\hline$e$ & Número de Euler \\
\hline$G$ & Conjunto de saídas da rede MLP \\
\hline$t_{k d}$ & Valor alvo da saída da rede \\
\hline$t_{k o}$ & Valor obtido da saída da rede \\
\hline$\nabla E$ & Gradiente do Erro \\
\hline$b$ & Valor de ativação \\
\hline$\eta$ & Taxa de aprendizagem \\
\hline
\end{tabular}


$\delta_{k} \quad$ Termo de erro de um neurônio da camada de saída

$\delta_{h} \quad$ Termo de erro de um neurônio da camada oculta

$\Delta w \quad$ Coeficiente de atualização de pesos

H Matriz de peso da camada oculta do modelo ELM

$H \dagger \quad$ Matriz generalizada inversa de Moore Penrose

T Dados de treinamento do modelo ELM

$\beta \quad$ Matriz dos pesos das conexões entre a camada oculta e a camada de saída

nh Número de neurônios na camada escondida

nt Número de elementos no conjunto de treinamento

I Matriz identidade 


\section{Sumário}

Introdução . . . . . . . . . . . . . . . . . 21

$1.1 \quad$ Considerações iniciais . . . . . . . . . . . . . . . . . . . . 21

1.2 Motivação e contextualização . . . . . . . . . . . . . . 21

$1.3 \quad$ Definição do problema . . . . . . . . . . . . . 23

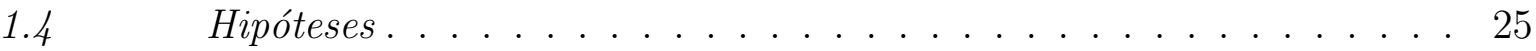

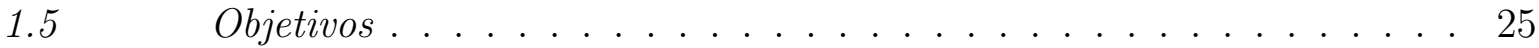

1.6 Estrutura da dissertação . . . . . . . . . . . . . . 26

3.1 Teorema de Bayes . . . . . . . . . . . . . . . . 30

3.2 Classificador Naïve Bayes . . . . . . . . . . . . . . . . . 32

3.3 Naive Bayes via máxima verossimilhança . . . . . . . . . . . . . . 32

3.4 Nä̈ve Bayes para dados continuos . . . . . . . . . . . . . 33

3.5 Nä̈ve Bayes via Maximização da Esperança . . . . . . . . . . . . . . 33

Aprendizado Sensível ao Custo . . . . . . . . . . 35

4.1 Abordagens sensiveis ao custo . . . . . . . . . . . . 36

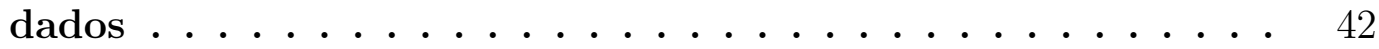

6.1 Máquinas ELM para dados desbalanceados . . . . . . . . . . 46

6.2 Discussão sobre o desempenho do modelo ELM . . . . . . . . . . 46

6.2.1 Críticas ao modelo ELM . . . . . . . . . . . . . 47

$7 \quad$ Avaliação de desempenho de classificadores . . . . . . . . . . 49

7.1 Métricas de Avaliação. . . . . . . . . . . . . . . 49

7.2 Avaliação de dados desbalanceados . . . . . . . . . . . . 50

7.3 Análise gráfica de desempenho no espaço $R O C \ldots \ldots \ldots$. . . . . . 51 
Resultados experimentais .............. 53

8.1

Modelo proposto . . . . . . . . . . . . . . . . 53

8.2

Experimentos Preliminares . . . . . . . . . . . . . . . 56

8.2.1 Descrição dos dados . . . . . . . . . . . . . . . 56

8.2.2 Adequação dos dados ao problema . . . . . . . . . . . . . 56

8.2.3 Experimentos de rotulação via PU . . . . . . . . . . . . . . . 57

8.2.4 Experimentos de classificação via ELM . . . . . . . . . . . . . . . 59

8.3 Experimentos com dados de Química Medicinal. . . . . . . . . . 62

8.3.1 Descrição dos dados . . . . . . . . . . . . . 62

8.3.2 Experimentos de rotulação via PU - Conjunto de dados AID1284 64

8.3.3 Experimentos de classificação via ELM - Conjunto de dados AID1284 65

8.3.4 Experimentos de rotulação via PU - Conjunto de dados AIDAID721 73

8.3.5 Experimentos de classificação via ELM - Conjunto de dados AID721 74

8.3.6 Experimentos de rotulação via PU - Conjunto de dados AID644 . 81

8.3.7 Experimentos de classificação via ELM - Conjunto de dados AID644 82

8.3.8 Experimentos de rotulação via PU - Conjunto de dados AID439 . . 89

8.3.9 Experimentos de classificação via ELM - Conjunto de dados AID439 90

8.3.10 Experimentos de rotulação via PU - Conjunto de dados AID1608 . 97

8.3.11 Experimentos de classificação via ELM - Conjunto de dados AID1608 98

8.3.12 Análise da estimação correta dos rótulos . . . . . . . . . . 106

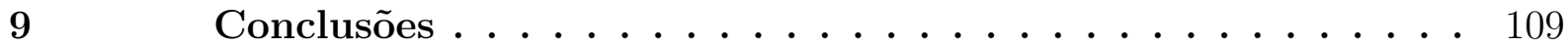

9.1 Contribuições e Trabalhos Futuros . . . . . . . . . . . . . . . 110

Referências $^{1} \ldots \ldots \ldots \ldots \ldots \ldots$

$\overline{1}$ De acordo com a Associação Brasileira de Normas Técnicas. NBR 6023. 


\section{Introdução}

\subsection{Considerações iniciais}

Desde o surgimento dos primeiros computadores, existe a expectativa de que estes fossem capazes de aprender com experiências e de solucionar problemas, como otimizar o consumo de energia de uma casa baseando-se nos padrões de seus ocupantes ou descobrir o tratamento mais eficiente para cada tipo de doença baseado em seus registros médicos, dentre outros objetivos. Ainda é um desafio fazer com que computadores aprendam conceitos da mesma forma que os humanos, considerando que esse avanço pode tornar possível novos usos do computador (MITCHELL, 1997b). Dessa forma, o processo de aprendizagem de máquina consiste em utilizar um algoritmo capaz de encontrar uma boa aproximação do conceito que se deseja aprender (BISHOP, 2006).

A Química Medicinal (QM) corresponde a uma subárea da Química que se dedica ao desenvolvimento de substâncias bioativas. Mais especificamente, a QM pode ser vista como uma área de pesquisa que envolve, em especial, a farmacologia e as suas pesquisas têm, como um dos objetivos, compreender os principais mecanismos relacionados com o desenvolvimento de uma doença alvo, de forma a propor novos compostos químicos candidatos a medicamentos (YOUNG, 2009).

Para desenvolver um novo medicamento, pesquisadores devem analisar os alvos biológicos de uma dada doença, descobrir e desenvolver candidatos a fármacos que atuem de forma eficiente no tratamento da mesma. Os pesquisadores devem também realizar testes biológicos em laboratório para validar a eficiência e os efeitos colaterais dos compostos desenvolvidos. Neste sentido, os algoritmos de aprendizagem de máquina assumem um papel importante no desenvolvimento de novos medicamentos, permitindo a automação de diversas etapas.

\subsection{Motivação e contextualização}

Dentre as diversas atividades existentes no processo de descoberta de novos medicamentos, há uma estratégia de análise de dados que propõe o estabelecimento de relações entre a estrutura química e a atividade biológica de uma série de moléculas (SAR) ${ }^{1}$. Com-

1 Do original, em inglês, Structure Activity Relationships. 
putacionalmente, esse processo pode ser aplicado a um conjunto de descritores obtidos por meio da análise realizada por programas específicos de QM como GAUSSIAN, SPARTAN e DRAGON (GERTRUDES, 2012).

Nessa etapa, são avaliados conjuntos de dados com observações (moléculas) e atributos (descritores químicos) contendo informações sobre as propriedades das substâncias candidatas a fármacos, as quais são analisadas com o auxílio de métodos computacionais. Essa análise é fundamental para relacionar grupos de substâncias químicas com diferenciados níveis de efeito (benéficos ou não) a um alvo biológico. Ou seja, nessa fase são identificados grupos de compostos químicos que podem ser utilizados no combate a uma determinada doença.

Nesse contexto, a busca por novos medicamentos pode ser vista como um problema de classificação de compostos com diferentes níveis de atividade biológica em relação a um determinado alvo no organismo. Dessa forma, técnicas de aprendizagem de máquina são importantes na análise dos dados de QM, pois com o auxílio desses métodos é possível descobrir padrões a partir de um conjunto de dados de forma rápida e econômica.

Diferente das aplicações convencionais, a aplicação de métodos de aprendizagem de máquina para análise de dados de QM necessita de uma atenção especial aos problemas relacionados à natureza dos dados analisados, como por exemplo, desbalanceamento de classes, dados incompletos, dificuldade de reconstrução de moléculas, incerteza na rotulação, dentre outros (VARNEK; BASKIN, 2012). Tais problemas tornam necessária a utilização de métodos específicos para tratá-los.

O trabalho de Varnek e Baskin (2012) apresenta seis desafios que os métodos de aprendizagem de máquina devem procurar solucionar. Estes são dispostos a seguir:

Natureza dos dados químicos: Os métodos de aprendizagem de máquina geralmente trabalham com vetores de dados de tamanho fixo. Esse formato também é aplicado nas análises de QM, nas quais os valores contidos nos vetores representam os descritores moleculares dos compostos em avaliação. Apesar de ser utilizada amplamente, essa representação vetorial não comporta todas as informações existentes na molécula, dificultando a reconstrução das mesmas, quando necessário, e resultando em perda de informação que poderia ser utilizada para melhorar o desempenho dos modelos de aprendizagem.

Problema de representatividade: Máquinas de aprendizado genéricas assumem que os dados de treinamento e teste derivam da mesma distribuição. No entanto, para os dados de QM o mesmo não acontece, pois os compostos sintetizados podem facilmente se afastar 
da distribuição dos compostos utilizados no treinamento, reduzindo consideravelmente a eficiência do modelo na fase de validação. Contudo, técnicas de adaptação de aplicabilidade do domínio podem ser utilizadas para reduzir esse problema (DAUM; MARCU, 2006).

Heterogeneidade e heterocedasticidade dos dados: O conjunto de treinamento pode ser oriundo de fontes de dados heterogêneas, refletindo na composição dos dados. Além disso, subconjuntos desses dados podem apresentar variações de erros diferentes. Essas características podem influenciar no funcionamento de máquinas estatísticas genéricas que consideram erros e distribuição de dados normais em seu processamento, invalidando diversos testes estatísticos.

Desbalanceamento de classes: A incidência maior de uma determinada classe em detrimento de outra, pode causar viés no processo de aprendizado em vários métodos de classificação. Para evitar que o desempenho dos modelos seja prejudicado, é indicado o uso de técnicas que tratem essa diferença para minimizar os impactos no processo de classificação.

Interpretabilidade dos modelos: Na análise de dados de QM, a interpretabilidade dos resultados recebe atenção especial. Quando existe essa possibilidade, o entendimento das interações moleculares e dos valores obtidos pelos modelos possibilita inferir sobre o comportamento de compostos semelhantes. Dessa forma, mesmo em modelos nos quais o processamento é feito de forma interna, as saídas podem apontar para propriedades específicas dos compostos envolvidos. Contudo, a complexidade envolvida na estrutura dos compostos não permite que o conhecimento aquirido em análises anteriores seja facilmente reaproveitado em outros estudos.

Incerteza na rotulação dos compostos inativos: Outra dificuldade encontrada no processo de descoberta de medicamentos é a incerteza na rotulação para os compostos definidos como inativos, já que o foco dos experimentos é encontrar os elementos biologicamente ativos. Esse problema impacta diretamente os métodos de classificação binária que dependem de exemplos de classes bem definidas em sua fase de treinamento.

\subsection{Definição do problema}

No processo de descoberta de medicamentos, um composto químico é considerado ativo para determinada doença alvo quando possui um grau suficiente de eficácia no 
tratamento desta doença, o que se reflete em uma variável resposta observada, denominada de nível de atividade biológica. O oposto também é verdadeiro, os compostos que apresentam nível baixo de atividade biológica para uma determinada doença pouco contribuem para o seu tratamento e, portanto, são considerados inativos. Entretanto, não existe um consenso sobre os valores de atividade biológica pré-definidos para considerar um composto químico pouco ativo ou muito ativo para uma doença específica. Esse cenário faz com que estudos produzam um pequeno grupo de exemplos com atividade alta, definidos como positivos, e os demais elementos com índices de atividade menores, um grupo maior, são definidos como negativos.

Em muitos casos, seja por custo de avaliação, tempo ou problemas de ordem técnica, nenhum estudo é efetuado para comprovar que a rotulação dos dados negativos é realmente confiável, podendo comprometer a eficiência da aplicação de modelos de aprendizado de máquina (VARNEK; BASKIN, 2012). Assim, no processo de descoberta de medicamentos, a presença de dados sem rotulação ou definidos com incerteza torna inadequada a abordagem clássica utilizada em tarefas de classificação supervisionada, para a qual é necessária a existência de exemplos bem definidos sobre as classes envolvidas, bem como de seus rótulos.

Neste trabalho, a técnica para tratar a incerteza na rotulação dos dados é baseada no aprendizado de dados positivos e não rotulados $(\mathrm{PU})^{2}$. Essa técnica consiste em uma abordagem de aprendizado semi-supervisionado que considera um grupo de elementos positivos e um grupo de elementos não rotulados para encontrar uma rotulação confiável para as instâncias do conjunto de treinamento (BHARDWAJ; GERSTEIN; LU, 2010). Para tratar o problema do desbalanceamento de classes, uma abordagem que utiliza Aprendizado Baseado na Sensibilidade do Custo (CSL) ${ }^{3}$ (ZADROZNY; LANGFORD; ABE, 2003) foi aplicada com o intuito de amenizar os efeitos causados pela presença de um número maior de elementos de uma determinada classe. Após essa etapa de rotulação preliminar, procede-se com a construção de classificadores para compostos no conjunto de teste utilizando, no caso do presente trabalho, a abordagem de Máquinas de Aprendizado Extremo $(\mathrm{ELM})^{4}$, que por sua vez, também será adaptada para lidar com o problema do

Do original, em inglês, Positive Unlabeled Learning.

3 Do original, em inglês, Cost-Sensitive Learning.

4 Do original, em inglês, Extreme Learning Machines. 
desbalanceamento de classes, que também será abordado na etapa de classificação dos compostos.

\subsection{Hipóteses}

Considerando o problema da incerteza na rotulação de dados de Química Medicinal, este trabalho pretende obter uma rotulação confiável de compostos químicos por meio da aplicação da técnica de aprendizado semi-supervisionado PU. Após a estimação dos novos rótulos, espera-se que classificadores construídos utilizando a abordagem ELM obtenham treinamento rápido e desempenho satisfatório na classificação de novos compostos.

Em relação ao cenário de desbalanceamento de classes, espera-se que por meio da análise sensível ao custo, seja possível controlar o comportamento dos modelos de estimação de rótulos e de classificação para que os mesmos não sofram viés devido à prevalência de uma determinada classe.

\subsection{Objetivos}

Este trabalho tem como objetivo geral aplicar o método de aprendizado PU para obter rótulos mais confiáveis para dados de treinamento e, assim, reduzir o impacto causado pela incerteza na rotulação de dados de QM. Uma vez que os novos rótulos forem estimados, este trabalho propõe ainda, a construção de classificadores para o mapeamento de cada instância (composto químico) no conjunto de teste, em uma das classes (ativo ou inativo), por meio da aplicação da aborgaem ELM. Além disso, para cada uma dessas etapas, os modelos devem também ser ajustados para tratar o problema do desbalanceamento das classes.

Para alcançar essa meta, os seguintes objetivos específicos devem ser atingidos:

- Implementar e aplicar a técnica de aprendizado semi-supervisionado PU para tratar o problema de incerteza na rotulação dos dados.

- Construir classificadores baseados na abordagem ELM, tendo como dados de treinamento as instâncias com rotulação estimada pela técnica PU.

- Ajustar os modelos de estimação de rótulos e de classificação para levar em consideração o desbalanceamento das classes. 
- Realizar experimentos de estimação de rótulos e de classificação com conjuntos de dados de QM.

- Analisar o desempenho dos modelos de classificação obtidos, comparando o cenário referente a dados de treinamento com rotulação original com outros gerados pela estimação de novos rótulos via aprendizado PU.

\subsection{Estrutura da dissertação}

Este trabalho está dividido em dez capítulos sendo a introdução o primeiro deles. O Capítulo 2 contempla a revisão bibliográfica, em que se discute os principais trabalhos desenvolvidos recentemente para tratar o problema de incerteza na rotulação dos dados de QM. No Capítulo 3, são descritos os conceitos inerentes ao aprendizado Bayesiano, em seguida, no Capítulo 4, é apresentado o conceito de aprendizado sensível ao custo, técnica utilizada neste trabalho para amenizar o impacto causado pelo desbalanceamento de classes. As técnicas apresentadas nos capítulos 3 e 4 são necessárias para o entendimento da técnica de aprendizado semi-supervisionado apresentada no Capítulo 5. No Capítulo 6, apresenta-se o uso de máquinas de aprendizado extremo, como uma alternativa computacionalmente barata e eficiente para a classificação de dados. O Capítulo 7 apresenta técnicas de avaliação de desempenho e discute técnicas utilizadas para este fim bem como seu comportamento frente a dados desbalanceados.

No capítulo 8 são apresentados os resultados do modelo aplicados a dados do conjunto Iris. Posteriormente, os resultados dos experimetos com dados de química medicinal. E, finalmente, no Capítulo 9, são apresentadas as conclusões e propostas de trabalhos futuros. 


\section{Revisão bibliográfica}

A incerteza na informação sobre a atividade biológica de compostos químicos é um problema recorrente no processamento de dados de QM. Nesse caso, os rótulos para os compostos inativos podem ter sido definidos sem a aplicação de um método de comprovação concreto, já que o foco dos experimentos é encontrar os elementos biologicamente ativos. Esse cenário impacta diretamente o uso de métodos de classificação binária que dependem de classes bem definidas (BASKIN; KIREEVA; VARNEK, 2010). Mesmo assim, uma série de técnicas podem ser aplicadas quando a rotulação dos dados não é a mais adequada. Neste caso, na presença de incerteza, a verificação da rotulação pode ser feita por algoritmos de aprendizagem de máquina que se baseiam na semelhança entre as moléculas e não em sua rotulação, fazendo com que seja necessária uma conversão dos compostos analisados em estruturas que possam ser comparadas entre si.

Normalmente, a classificação de dados para os quais só existe informação sobre uma das classes envolvidas, se baseia na distribuição de frequência da classe conhecida. Dessa forma, esses métodos consideram as instâncias que se comportam de maneira incomum como anormalidades, identificando os exemplos que não fazem parte do conjunto. Essa abordagem indutiva depende diretamente da distribuição dos dados, apresentando resultados insatisfatórios na presença de dados contaminados ou não rotulados. O processo semi-supervisionado de detecção de mudanças $^{1}$, proposto em (BLANCHARD, 2010), propõe um método de classificação estatisticamente consistente, que considera os elementos sem rotulação para construir uma regra de decisão capaz de classificar pontos arbitrários em problemas relacionados à classificação Neyman-Pearson.

Em (KRAWCZYK; WO, 2012), é proposto o uso de diversas técnicas de classificação que consideram uma classe, assim podem ser considerados vários tipos de entradas e os métodos envolvidos poderiam se ajudar mutuamente efetuando a busca por solução em espaços de hipóteses diferentes. Um problema evidente nessa abordagem é o custo necessário para lidar com todas as particularidades de cada modelo simultaneamente.

O processo de classificação proposto em (KARPOV et al., 2011), baseia-se no conceito de que estruturas semelhantes possuem características semelhantes. Assim, a partir de compostos com atividade biológica conhecida, os compostos não rotulados são classificados efetuando uma verificação de similaridade baseada em índices como Jaccard

$\overline{1}$ Do original, em inglês, Semi-Supervised Novelty Detection. 
ou Tanimoto (FERREIRA, 2004). Esse método foi aplicado no processo de verificação virtual $^{2}$ de compostos baseado em ligantes na seleção de substâncias candidatas a novos fármacos. Uma desvantagem desse método é a alta sensibilidade a mudanças nos valores dos descritores químicos, na qual uma pequena variação nos valores pode ocasionar mudanças representativas no conjunto de estruturas selecionadas.

Outra abordagem que promete tempo menor na fase de treinamento são os métodos baseados em grafos. Esses métodos, que também trabalham com busca de similaridade entre as moléculas, necessitam que as mesmas sejam convertidas em grafos e, após a conversão, são efetuados processos de mineração de dados e busca para verificar o quão similar é a instância observada em relação às demais. Essa forma de avaliação se concentra principalmente nas informações estruturais das moléculas ao invés de seus rótulos (RALAIVOLA et al., 2005).

Nesse tipo de análise, a estrutura molecular é representada como um grafo possuindo um mapeamento dos átomos de seus elementos químicos. Essa forma de representação torna possível a verificação da similaridade entre as estruturas e pode ser submetida a modelos estatísticos ou a algoritmos de aprendizagem para a análise de suas características (LUSCI; POLLASTRI; BALDI, 2013).

A técnica de kernel de grafos ${ }^{3}$ utiliza representação de moléculas por meio de grafos de caminhos identificados. Essa abordagem torna possível a aplicação de técnicas de busca em profundidade e contagem de caminhos identificados (RALAIVOLA et al., 2005). Nesse formato, também é possível a obtenção de medidas escalares que podem ser utilizadas em casos em que os rótulos não são conhecidos, possibilitando a criação de produtos escalares e uso de abordagens como máquinas de vetores suporte (VISHWANATHAN et al., 2010).

O processo de mineração de grafos moleculares por meio de Redes Neurais Artificiais (RNA), permite o acesso às propriedades moleculares de forma direta, sem a necessidade de processamento de seus descritores (NASRI et al., 2011). O método em questão recebe como treinamento as propriedades que se deseja investigar e utiliza uma arquitetura de RNA inspirada no modelo de visão biológico em seu processamento. Essa abordagem obteve resultados expressivos na análise de viscosidade e ponto de evaporação de hidrocarbonetos, polarização de moléculas, dentre outros (NASRI et al., 2011).

2 Do original, em inglês, Virtual Screening.

3 Do original, em inglês, Graph kernels. 
A classificação de grafos baseada em padrões de recorrência ${ }^{4}$ é um processo que utiliza a comparação de subgrafos para a classificação de compostos químicos. Esse método identifica padrões nas moléculas por meio do agrupamento das ocorrências estruturais semelhantes. Tal técnica apresentou resultados expressivos com relação à acurácia e tempo de execução (YOUNG, 2009).

O método de $k$ relações de vizinhos mais próximos ${ }^{5}(k$-RNN) possibilita a análise de grandes volumes de dados, sem a necessidade de transformação tabular. Como um tipo de mineração de múltiplas relações, essa técnica permite que os relacionamentos entre os dados sejam extraídos e as análises sejam feitas de forma indutiva, permitindo encontrar quais demais objetos podem estar relacionados com a instância a ser classificada (FONSECA; COSTA, 2008).

Os métodos baseados em grafos oferecem uma alternativa à análise de compostos químicos por meio da investigação de sua estrutura molecular, tornando possível a aplicação de técnicas de similaridade e identificação de padrões. Essa abordagem reduz o impacto da incerteza na rotulação já que a análise é feita com foco na estrutura do grafo gerado e não na rotulação. Mesmo assim, a modelagem de compostos químicos para o formato de grafos não é trivial (RALAIVOLA et al., 2005).

Sendo assim, o presente trabalho apresenta uma proposta de análise de substâncias bioativas frente a um determinado alvo biológico, na qual os dados com incerteza na rotulação são inicialmente tratados e em seguida utilizados para a construção de um classificador eficiente e com tempo de treinamento menor. Uma abordagem de tratamento dos dados similar foi aplicada em (BHARDWAJ; GERSTEIN; LU, 2010) para predição de proteínas periféricas em dados de origem genômica.

4 Do original, em inglês, Graph Classification Based on Pattern Co-occurrence.

5 Do original, em inglês, k-Relational Nearest Neighbour. 


\section{Aprendizado Bayesiano}

Para a execução de tarefas de classificação, é possível utilizar métodos baseados na teoria do aprendizado Bayesiano. Esses métodos constroem um modelo probabilístico que utiliza conhecimento prévio e informações extraídas dos dados observados para determinar a probabilidade final de uma hipótese estar correta. Mais especificamente, os algoritmos que se baseiam nesse tipo de aprendizado trabalham com o cálculo explícito de probabilidades, sendo que qualquer conhecimento prévio sobre as hipóteses pode ser combinado com informações extraídas dos dados observados, contribuindo para o aumento ou redução da confiança de que a hipótese avaliada esteja correta.

Uma das vantagens dos algoritmos baseados em aprendizado Bayesiano é a possibilidade de trabalhar com hipóteses por meio de previsões probabilísticas, permitindo uma abordagem mais flexível do que a completa eliminação da hipótese quando a mesma se mostra inconsistente com algum exemplo no conjunto de dados.

Neste trabalho, um método baseado em aprendizado Bayesiano será utilizado como parte do processo da rotulação de compostos químicos, estimando a probabilidade desses elementos serem classificados como ativos ou inativos.

\subsection{Teorema de Bayes}

Em problemas de classificação envolvendo um número $k$ de classes, o objetivo consiste em determinar, para uma nova instância, a classe mais provável, dado o conjunto de treinamento $\boldsymbol{D}$.

Segundo as regras do aprendizado Bayesiano, estimar as probabilidades das hipóteses pela observação de dados de exemplos contribui para uma tomada de decisão ótima, no sentido de descobrir a melhor hipótese existente em um espaço de hipóteses $\boldsymbol{V}$, observandose os dados de treinamento $\boldsymbol{D}$. Nesse sentido, a probabilidade de cada hipótese pode ser influenciada por conhecimento prévio ou por informações extraídas dos dados observados, uma vez que cada instância presente no conjunto de treinamento influencia na medida de confiança da hipótese mais provável.

A base do aprendizado Bayesiano é o Teorema de Bayes, que afirma que a busca pela melhor hipótese consiste em encontrar a hipótese mais provável em relação ao conjunto $\boldsymbol{D}$, calculando as seguintes probabilidades: 
- A probabilidade a priori $P(v)$, que corresponde à probabilidade inicial de uma hipótese $v$ antes da observação dos dados de treinamento $\boldsymbol{D}$. Essa probabilidade reflete também qualquer conhecimento referente à chance que $v$ possui de ser a hipótese correta, antes que os dados tenham sido observados.

- A probabilidade a priori $P(\boldsymbol{D})$, que representa a probabilidade do conjunto de treinamento $\boldsymbol{D}$ ser observado.

- A probabilidade $P(\boldsymbol{D} \mid v)$, que denota a probabilidade de se observar o dado D, apresentado para treinamento, considerando a situação na qual a hipótese $v$ é verdadeira.

- A probabilidade $P(v \mid \boldsymbol{D})$, denominada de probabilidade a posteriori, que corresponde à confiança em que uma hipótese $v$ é verdadeira após a observação do conjunto de treinamento $\boldsymbol{D}$.

O Teorema de Bayes propõe uma forma de calcular a probabilidade a posteriori $P(v \mid \boldsymbol{D})$ utilizando a probabilidade a priori $P(v)$, que é independente dos dados de treinamento, e a probabilidade condicional $P(\boldsymbol{D} \mid v)$ dos dados serem observados em relação à determinada hipótese. Tal teorema é dado por:

$$
P(v \mid \boldsymbol{D})=(P(\boldsymbol{D} \mid v) P(v)) /(P(\boldsymbol{D})) .
$$

Dado um conjunto de hipóteses $V$, a hipótese com maior grau de confiança de ser verdadeira é denominada de hipótese maximum a posteriori e também pode ser calculada utilizando o Teorema de Bayes, conforme a seguinte equação:

$$
\begin{aligned}
v_{M A P} & =\underset{v \in \mathbf{V}}{\operatorname{argmax}} P(v \mid \mathbf{D}) \\
& =\underset{v \in \mathbf{V}}{\operatorname{argmax}} \frac{P(\mathbf{D} \mid v) P(v)}{P(\mathbf{D})} \\
& =\underset{v \in \mathbf{V}}{\operatorname{argmax}} P(\mathbf{D} \mid v) P(v)) .
\end{aligned}
$$

É importante ressaltar que na equação apresentada anteriormente, a probabilidade $P(\boldsymbol{D})$ é omitida por ser uma constante independente de $v$. Neste trabalho, estimar a rotulação correta para os elementos de um conjunto consiste em encontrar a hipótese mais provável em um universo de hipóteses $\mathbf{V}=\left\{v_{1}, v_{2}\right\}$, no qual $v_{1}$ corresponde à hipótese de um composto químico ser biologicamente ativo e $v_{2}$ corresponde à hipótese deste composto ser biologicamente inativo. 


\subsection{Classificador Nä̈ve Bayes}

O classificador Naïve Bayes (NB), baseado no modelo de aprendizado bayesiano, utiliza cálculos de estimativas de probabilidades para encontrar a classe mais provável para uma instância. Neste caso, um conjunto de treinamento $\boldsymbol{D}$, contendo exemplos representados por vetores de características $<a_{1}, a_{2}, \ldots, a_{n}>$ será utilizado para a obtenção de uma função alvo capaz de associar novas instâncias a uma classe no conjunto finito $\mathbf{V}=\left\{v_{1}, v_{2}, \ldots v_{n}\right\}$. Mais especificamente, o classificador NB tem a finalidade de atribuir uma classe a uma nova instância submetida à análise calculando a hipótese mais provável $v_{\mathrm{NB}}$, dados os valores que representam o novo exemplo, conforme formulado na equação:

$$
v_{N B}=\underset{v_{j} \text { inV }}{\operatorname{argmax}} P\left(a_{1}, a_{2} \ldots a_{n}\right) P\left(v_{j}\right)
$$

Entretanto, estimar as diferentes probabilidades a posteriori considerando a disposição dos atributos requer um conjunto de treinamento significantemente grande. Dessa forma, o classificador NB assume ingenuamente que os atributos são condicionalmente independentes e que a probabilidade de observar a conjunção $\left(a_{1}, a_{2} \ldots a_{n}\right)$ corresponde ao produto de suas probabilidades individuais, o que pode ser descrito da seguinte forma:

$$
v_{N B}={ }_{v_{j} i n V}^{\operatorname{argmax}} P\left(v_{j}\right) \prod_{i} P\left(a_{i} \mid v_{j}\right) .
$$

\subsection{Naive Bayes via máxima verossimilhança}

Considerando que os dados de treinamento estão rotulados, os parâmetros $P\left(v_{j}\right)$ e $P\left(a_{i} \mid v_{j}\right)$ podem ser estimados a partir do conjunto de exemplos $\left(d_{i}, v_{j}\right)$, para $i=1, \ldots, n$, em que $n$ representa o número de exemplos disponíveis no conjunto, e $v_{j}$ corresponde ao rótulo associado à instância $d_{i}$. Nesse caso, é possível utilizar estimativas obtidas por máxima verossimilhança ${ }^{1}$ (ML) para encontrar os parâmetros do modelo (MITCHELL, 1997b). Assim, a probabilidade $P\left(v_{j}\right)$ pode ser obtida da seguinte forma:

$$
P\left(v_{j}\right)=\frac{\left(\operatorname{total}\left(v_{j}\right)\right)}{n},
$$

em que $\operatorname{total}\left(v_{j}\right)$ corresponde ao número de exemplos rotulados com $v_{j}$ no conjunto de treinamento. Para estimar a probabilidade $P\left(a_{i} \mid v_{j}\right)$, é necessário ainda contar o número 
de vezes em que um determinado rótulo $v_{j}$ é visto em conjunto com um determinado valor de atributo $a_{i}$ :

$$
P\left(a_{i} \mid v_{j}\right)=\frac{\left(\operatorname{total}\left(a_{i} \mid v_{j}\right)\right)}{\left(\operatorname{total}\left(v_{j}\right)\right)}
$$

\subsection{Naïve Bayes para dados contínuos}

Nos casos nos quais o classificador Bayesiano é empregado na avaliação de dados contínuos, deve-se encontrar uma outra forma de se obter as probabilidades envolvidas no funcionamento deste tipo de classificador. Uma possibilidade é assumir que a distribuição de probabilidade para cada atributo é gaussiana, ou seja, pode ser definida pela média e o desvio padrão para cada atributo em relação a cada uma das classes (MITCHELL, 1997a). Dessa forma, durante a etapa de treinamento de um classificador Bayesiano a média pode ser obtida da seguinte forma:

$$
\mu_{i k}=\frac{1}{\sum_{j} \delta\left(\mathbf{V}^{j}=v_{k}\right)} \sum_{j} \mathbf{X}_{i}^{j} \delta\left(\mathbf{V}^{j}=v_{k}\right),
$$

em que $\mathbf{X}_{i}$ representa o $i$-ésimo valor contínuo de entrada da coluna de atributos $\mathbf{X}$ e

$v_{k}$ a k-ésima classe existente em V. Posteriormente, o desvio padrão pode ser calculado conforme a seguinte fórmula:

$$
\sigma_{i k}^{2}=\frac{1}{\left(\sum_{j} \delta\left(\mathbf{V}^{j}=v_{k}\right)\right)-1} \sum_{j}\left(\mathbf{X}_{i}^{j}-\mu_{i k}\right)^{2} \delta\left(\mathbf{V}^{j}=y_{k}\right)
$$

\subsection{Naïve Bayes via Maximização da Esperança}

O algoritmo de Maximização da Esperança ${ }^{2}$ (EM) consiste em um método iterativo que tem a finalidade de estimar parâmetros em modelos probabilísticos para dados incompletos (DEMPSTER; LAIRD; RUBIN, 1977). No contexto deste trabalho, situação semelhante pode ser identificada em relação à ausência de rotulação nos dados de Química Medicinal.

O algoritmo EM, em cada uma de suas iterações, estima uma série de configurações para os parâmetros $\theta_{0}, \theta_{1}, \ldots, \theta_{m}$, em que $m$ denota o número de iterações estipuladas pelo

2 Do original, em inglês, Expectation Maximization. 
usuário. Neste trabalho, os parâmetros $\theta$, fornecerão os valores para a função alvo que classifica os compostos em ativos e inativos, sendo que, os parâmetros $\theta_{0}$, correspondem aos valores iniciais de probabilidade a priori de cada uma das classes possíveis e às probabilidades condicionais dos valores dos atributos do conjunto de treinamento em relação às classes.

Como primeiro passo em cada iteração, (Passo E), deve-se calcular as probabilidades a posteriori $P\left(v_{j} \mid d_{i}\right)$ para cada um dos exemplos $d_{i}$. O valor obtido pelo cálculo de $P\left(v_{j} \mid d_{i}\right)$ corresponde à probabilidade condicional para o rótulo $v_{j}$ para o $i$-ésimo exemplo, dados os parâmetros $\theta^{\mathbf{t}-\mathbf{1}}$ :

$$
P\left(v_{j} \mid d_{i} ; \theta^{t-1}\right)=\frac{P^{t-1}\left(v_{j}\right) \prod_{j=1}^{k} p^{t-1}\left(a_{i} \mid v_{j}\right)}{\sum_{j=1}^{k} P^{t-1}\left(v_{j}\right) \prod_{j=1}^{k} P_{j}^{t-1}\left(a_{i} \mid v_{j}\right)}
$$

No segundo passo, (Passo M), as probabilidades condicionais das instâncias em relação às classes calculadas anteriormente são utilizadas para calcular os novos valores dos parâmetros representados por $\theta$ :

$$
\begin{gathered}
P\left(v_{j}\right)=\frac{1}{n} \sum_{i=1}^{n} P\left(v_{j} \mid d_{i}\right), \\
P\left(a_{i} \mid v_{j}\right)=\frac{\sum_{j=1}^{k} P\left(v_{j} \mid d_{i}\right)}{\sum_{i} P\left(v_{j} \mid d_{i}\right)}
\end{gathered}
$$

Esse processo se repete iterativamente até que ocorra a convergência dos parâmetros estimados $\theta$, observando se seus valores não se alterem significativamente entre duas iterações consecutivas. 


\section{Aprendizado Sensível ao Custo}

Os algoritmos responsáveis por executar tarefas de classificação têm como objetivo atribuir corretamente a um objeto uma das classes possíveis para o problema. Mais especificamente, no intuito de identificar a hipótese correta, essas técnicas destinam-se a reduzir o erro no processo de identificação do rótulo de novas instâncias (KONG; NG; ZHOU, 2013). No que diz respeito às abordagens tradicionais, os algoritmos consideram que os custos dos erros de classificação para cada classe são relativamente próximos. Todavia, essa suposição não é válida em cenários de desbalancemaneto de dados, pois a diferença na distribuição das classes pode acarretar em um funcionamento não adequado do modelo (WEISS; PROVOST, 2003). Essa situação é agravada principalmente quando o erro de classificação da classe minoritária possui um impacto significante no domínio analisado (BATISTA; PRATI; MONARD, 2004).

Na tentativa de amenizar o impacto causado pela disparidade entre o número de instâncias em cada classe, alguns recursos podem ser adotados. Dentre estes pode-se citar :

- Alteração da distribuição de classes: Nesta categoria, o objetivo principal é promover o equilíbrio entre as classes. Com essa finalidade, são aplicados recursos como sobreamostragem $^{1}$, que consiste na adição de mais exemplos da classe minoritária ao conjunto de treinamento com o propósito de reduzir o impacto causado pela prevalência da classe majoritária (LING; SHENG, 2008). Por outro lado, também podem ser aplicados procedimentos de subamostragem ${ }^{2}$, técnica que se concentra na remoção de instâncias pertencentes à classe majoritária, reduzindo assim a disparidade entre as classes (PEREIRA et al., 2013).

- Alteração do limiar de classificação: Nessa abordagem, o limiar utilizado para classificação é modificado em benefício da classe de menor distribuição (SHENG; LING, 2006).

As abordagens relacionadas à amostragem dependem diretamente da disponibilidade de exemplos para treinamento. Quando isso não é possível, métodos que modificam o processo de análise do limiar são mais adequados. Assim, o Aprendizado Baseado na Sensibilidade do Custo $(\mathrm{CSL})^{3}$ consiste em uma forma de penalizar o erro de classificação 
em relação a uma determinada classe, (ZADROZNY; LANGFORD; ABE, 2003) reduzindo os efeitos causados pelo desbalanceamento.

Nesse tipo de análise, a penalização do erro é feita com o auxílio de uma matriz de custos que utiliza a notação $C(i, j)$ indicando o custo do erro de classificação da classe esperada $j$ em relação à classe predita $i$ (SCHIERZ, 2009).

Tabela 1 - Matriz de Custos

\begin{tabular}{|c|c|c|}
\hline & Esperado Positivo & Esperado Negativo \\
\hline Predito Positivo & $C(0,0)$ & $C(0,1)$ \\
\hline Predito Negativo & $C(1,0)$ & $C(1,1)$ \\
\hline
\end{tabular}

A matriz de custos $\boldsymbol{C}$ pode ser obtida de várias maneiras. Em alguns trabalhos, utiliza-se métodos empíricos (SCHIERZ, 2009), variando os custos dentro de uma faixa de valores, de modo a produzir vários modelos, que são posteriormente ordenados de acordo com seu desempenho. Outra abordagem baseia-se no uso da quantidade de elementos da classe majoritária como custo ou até mesmo, no uso da razão entre o número de elementos positivos em relação à quantidade de elementos negativos (SEO; SYCARA, 2006).

\subsection{Abordagens sensiveis ao custo}

A matriz de custos pode ser incorporada a diversas técnicas que se destinam ao ajuste dos dados desbalanceados, sugerindo uma adaptação de algoritmos já conhecidos (LING; SHENG, 2008). Essa prática mostra-se relevante no contexto deste trabalho, pois propõe ajustes no algoritmo utilizado sem interferir na distribuição das classes. Dentre as abordagens CSL mais utilizadas, pode-se citar o método de limiarização ${ }^{4}$ e metaaprendizagem sensível ao custo (MCSL) ${ }^{5}$, descritos sucintamente a seguir.

A técnica de limiarização (SHENG; LING, 2006) sugere que sejam feitos ajustes no limiar de classificação, no sentido de valorizar os elementos pertencentes a classe minoritária. Respeitando a seguinte desigualdade,

$$
P(v=0 \mid x) c_{10}+P(v=1 \mid x) c_{11} \leq P(v=0 \mid x) c_{00}+P(v=1) c_{01},
$$

4 Do original, em inglês, Thresholding

5 Do original, em inglês, Meta-Cost Sensitive Learning 
em que $v=0$ corresponde à hipótese positiva e $v=1$ indica a hipotese negativa. Considerando uma matriz de custos conhecida e uma tomada de decisão ótima (ZADROZNY; LANGFORD; ABE, 2003), um limiar pode ser obtido da seguinte forma:

$$
t^{*}=\frac{C(0,1)+C(1,1)}{C(0,1)+C(1,0)-C(0,0 \mid)-C(1,1)} .
$$

Posteriormente, o limiar $t^{*}$ é utilizado para classificar cada instância, considerando as probabilidades iguais ou acima desse valor como positivas, caso contrário, como negativas. Outra possibilidade é efetuar a busca por um limiar adequado por meio de validação cruzada (LING; SHENG, 2008), sendo que o limiar que obtiver menor erro de classificação é o escolhido.

Neste trabalho, a matriz de custos é desconhecida, impossibiliando o cálculo do limiar. Dessa forma, a análise MCSL é utilizada para lidar com o problema de incerteza por meio da inserção de custo de erro de classificação no modelo utilizado (DOMINGOS, 1999). Considerando que não existem riscos para a classificação correta de um exemplo, as posições de acerto na matriz de custos (ver tabela 1) não são penalizadas. Por outro lado, o custo de classificação incorreta é utilizado como fator de ponderação na obtenção dos riscos de classificação existentes para cada exemplo, em relação às suas possíveis hipóteses. Seja $P(j \mid x)$ a probabilidade condicional do valor $\mathrm{x}$ de um atributo pertencer a classe $\mathrm{j}$ e $\mathrm{C}(\mathrm{i}, \mathrm{j})$ o custo de se classificar um uma intância da classe esperada j em relação a classe predita i, o risco, conhecido como Risco de Bayes, pode ser obtido da seguinte forma:

$$
R(i \mid x)=\sum_{j} P(j \mid x) C(i, j) .
$$

Essa abordagem define como hipótese mais provável para cada exemplo aquela que possui menor risco, atenuando os efeitos causados pelo desbalanceamento na aplicação de classificadores tradicionais.

Neste trabalho, o conceito de MCSL é aplicado no processo de estimação da rotulação, executado pelo método de classificação semi-supervisionado, a ser descrito no próximo capítulo. 


\section{Aprendizado de dados positivos e não rotulados (PU)}

$\mathrm{Na}$ análise de dados de Química Medicinal, além do fato de exemplos da classe positiva serem encontrados com menor frequência do que os da classe negativa, conjuntos com instâncias sem rotulação também são comuns. Esse cenário torna inadequado o uso de técnicas de classificação supervisionadas tradicionais, que necessitam de uma quantidade significativa de exemplos de cada uma das classes envolvidas.

A técnica de Aprendizado de Dados Positivos e não Rotulados (Aprendizado PU) trata o problema da incerteza e da ausência de rótulos por meio de uma abordagem de classificação parcialmente supervisionada, na qual dados rotulados e não rotulados são considerados no processo de construção de um classificador.

No aprendizado PU, um conjunto $\boldsymbol{X}$, contendo dados rotulados e não rotulados, é dividido em outros subconjuntos, como descrito a seguir:

- Conjunto $\boldsymbol{P}$, denominado conjunto positivo, consiste no grupo de elementos com rotulação conhecida. Neste trabalho, o conjunto $\boldsymbol{P}$ corresponde ao grupo de elementos que possuem atividade biológica positiva já conhecida para uma determinada doença.

- O conjunto $\boldsymbol{U}$, que é formado pelos elementos presentes em $\boldsymbol{X}$ após a retirada dos elementos em $\boldsymbol{P}$. Este conjunto possui somente os elementos de atividade biológica não conhecida, ou seja, todas as instâncias não rotuladas.

- O conjunto $\boldsymbol{S}$, denominado conjunto de espiões, é formado por uma porcentagem, tipicamente 15\%, dos elementos do conjunto $\boldsymbol{P}$ (LIU et al., 2002).

- Os elementos do conjunto dos espiões $\mathbf{S}$ são agrupados com os elementos não rotulados $\boldsymbol{U}$, formando o conjunto $\boldsymbol{U} \boldsymbol{S}$.

Após a obtenção dos conjuntos iniciais, os elementos de rotulação conhecida, representados por $\boldsymbol{P}$, são associados à classe positiva (compostos ativos), e as instâncias do conjunto $\boldsymbol{U S}$, contendo os elementos sem rotulação juntamente com espiões $\boldsymbol{S}$, são inicialmente associados à classe que define os elementos negativos (compostos inativos).

Os conjuntos $\boldsymbol{P}$ e $\boldsymbol{U S}$, agora rotulados respectivamente como positivos e negativos, são submetidos a um método baseado no algoritmo EM, denominado de EM Inicial (I-EM), com o objetivo de estimar a rotulação dos elementos pertencentes a $\boldsymbol{U} \boldsymbol{S}$.

O método I-EM utiliza duas fases de aplicação do classificador NB para calcular as probabilidades a posteriori dos elementos do conjunto $\boldsymbol{U S}$. O primeiro classificador 
NB é construído de maneira não iterativa utilizando a técnica de estimação descrita na seção 3.4. Dessa forma, inicialmente são definidas as probabilidades de classes iniciais para as instâncias do conjunto $\boldsymbol{U S}$. Em seguida, o segundo classificador NB calcula as novas probabilidades a posteriori para $\boldsymbol{U} \boldsymbol{S}$ de forma iterativa utilizando o algoritmo I-EM (ver seção 3.5) até que as probabilidades condicionais das instâncias desse conjunto não se alterem significativamente entre duas iterações consecutivas.

Após a execução do algoritmo I-EM, o conjunto $\boldsymbol{U} \boldsymbol{S}$ possui uma nova rotulação, sendo que os elementos de $\boldsymbol{S}$, conjunto dos espiões, permanecem com a rotulação inicial conhecida como positiva. Dessa forma, essa fase do aprendizado $\boldsymbol{P} \boldsymbol{U}$ dedica-se a descobrir elementos confiavelmente negativos, neste trabalho denominados $\boldsymbol{R} \boldsymbol{N}$, no intuito de destacar exemplos bem definidos no grupo dos negativos.

Para compor o conjunto $\boldsymbol{R} \boldsymbol{N}$, define-se um limiar $t$, de modo que as instâncias $x_{i}$ para as quais as probabilidades $P\left(\right.$ positivo $\left.\mid x_{i}\right)$ são menores que $t$ são consideradas como confiavelmente negativas e, consequentemente incorporadas ao conjunto $\boldsymbol{R} \boldsymbol{N}$.

Figura 1 - Subdivisão de conjunto no Aprendizado PU - O conjunto de treinamento $\boldsymbol{X}$ possui todos os elementos $x_{i}$ rotulados e não rotulados. Em seguida, o conjunto dos elementos positivos, aqui identificados como $\boldsymbol{P}$, é separado dos elementos do conjunto $\boldsymbol{U}$ que corresponde ao conjunto não rotulado. Uma parte do conjunto $\boldsymbol{P}$ denominada de espiões $\boldsymbol{S}$, é agrupada ao conjunto $\boldsymbol{U}$. Em seguida, o algoritmo I-EM se encarrega de fornecer uma nova rotulação aos elementos, identificando as instâncias com rotulação confiavelmente negativa incluídas em um novo conjunto identificado por $\boldsymbol{R} \boldsymbol{N}$.

\section{Positivos (P) Não Rotulados (U) Wegativos Confiáveis (RN)}

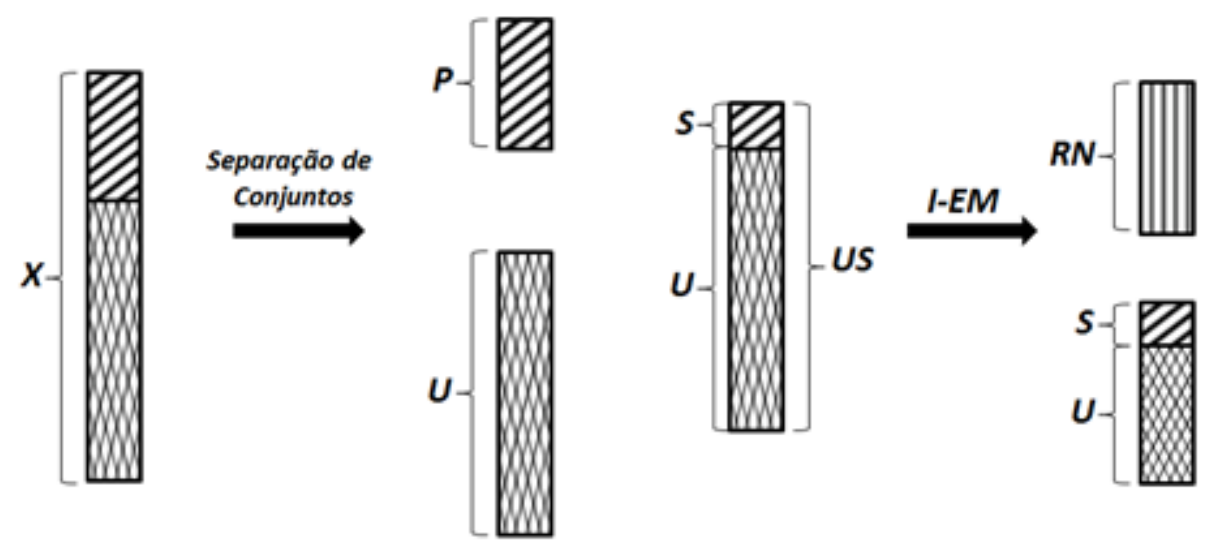

Fonte: João Carlos Silva de Souza (2015). 
Mais especificamente, considerando o conjunto de espiões $\mathbf{S}=s_{1}, s_{2}, \ldots s_{k}$ e a probabilidade condicional com relação a classe positiva para cada um deles, $P\left(\right.$ positivo $\left.\mid s_{i}\right)$, pode-se fixar o limiar $t$ como sendo a menor probabilidade condicional existente nesse conjunto $t=\min \left\{P\left(\right.\right.$ positivo $\left.\mid s_{1}\right), P\left(\right.$ positivo $\left.\mid s_{2}\right) \ldots, P\left(\right.$ positivo $\left.\left.\mid s_{k}\right)\right\}$. É relevante mencionar que para dados com ruído ou com valores atípicos, a escolha de um valor de probabilidade muito pequeno ou até mesmo igual a zero como limiar pode causar uma separação incorreta do conjunto. Para reduzir o impacto desse problema, é aconselhável trabalhar com controle de ruído, estabelecendo uma porcentagem mínima de quantos elementos devem estar abaixo da probabilidade escolhida. Geralmente esse valor é especificado em torno de $15 \%$.

\subsection{Adaptação do PU para o problema de desbalanceamento}

O aprendizado PU é empregado neste trabalho com o objetivo de tratar o problema da incerteza na rotulação dos dados de treinamento. Entretanto, devido à presença de classes desbalanceadas, o modelo apresenta viés em relação à classe com maior número de elementos. Assim, considerando a técnica MCSL já mensionada na sessão 4.1, propõese, neste trabalho, que o modelo PU seja modificado para adotar o risco de Bayes em detrimento da utilização do cálculo das probabilidades, tal como formulado no modelo original. 


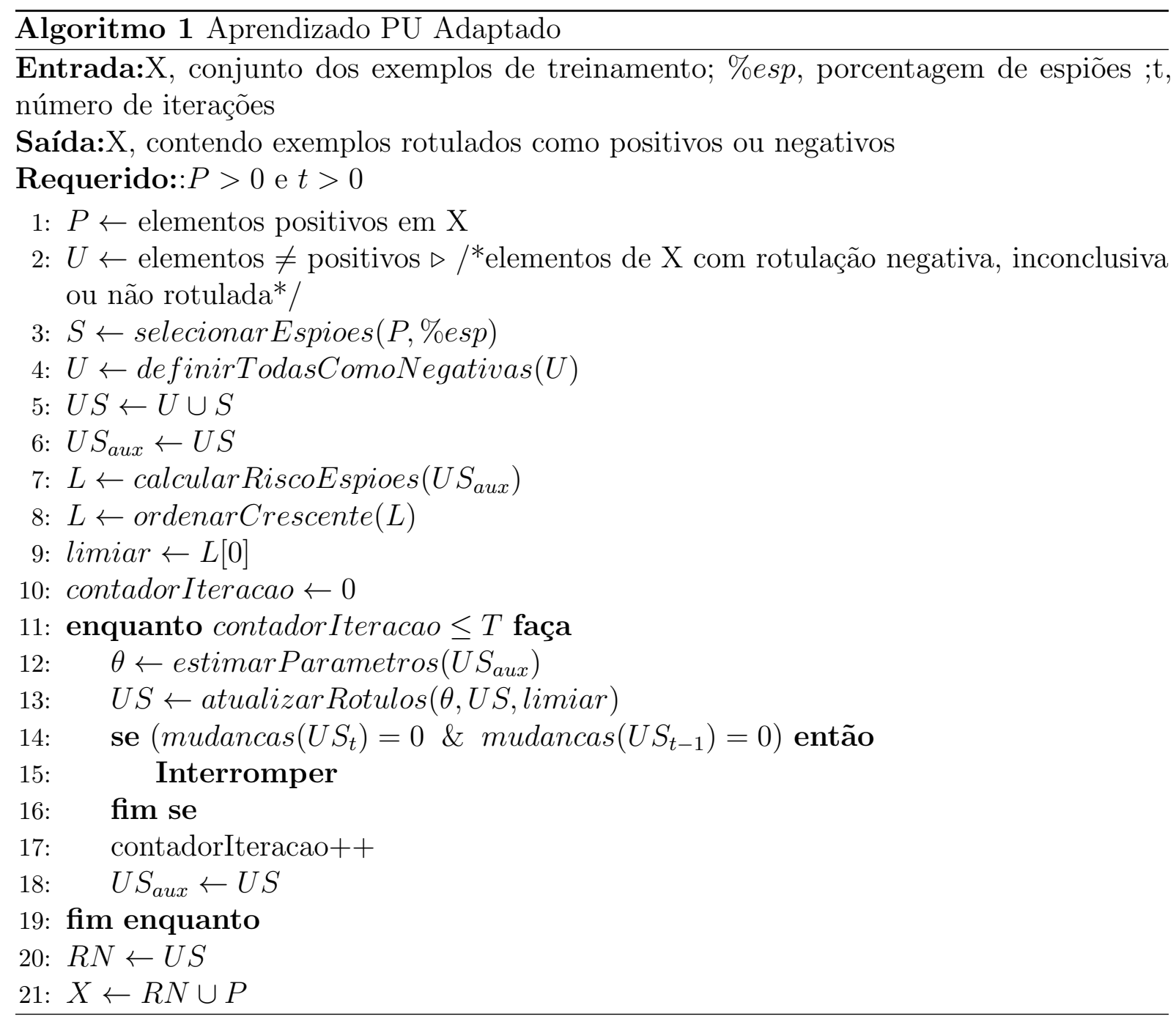

No algoritmo 1, o cálculo no risco foi inserido para atenuar o cenário de desbalanceamento de classes. Dessa forma, após a aplicação da técnica de aprendizado PU, tem-se um problema clássico de classificação, em que é possível considerar uma técnica de aprendizado supervisionado utilizando como exemplos de treinamento os elementos do conjunto $\boldsymbol{P}$, rotulados como positivos, e os elementos do conjunto $\boldsymbol{R} \boldsymbol{N}$, rotulados como negativos. 


\section{Máquinas de aprendizado extremo (ELM) para classificação de dados}

As máquinas ELMs consistem em um modelo de aprendizagem de máquina baseados no paradigma conexionista que se propõe a manter a capacidade de aproximação universal das redes MLP, reduzindo o tempo da fase de treinamento.

Apesar das vantagens obtidas na aplicação das redes MLP na resolução de problemas de classificação, como um aproximador universal com alto grau de generalização (MITCHELL, 1997b), esses modelos apresentam dificuldades com relação ao tempo de treinamento, além de necessitar de uma quantidade significativa de exemplos para essa fase.

O desenvolvimento do algoritmo BP tornou viável a aplicação de redes feedforward para a resolução de vários problemas do mundo real. Mesmo assim, devido à natureza da otimização do modelo, que é feita via gradiente descendente, o modelo apresenta problemas de convergência lenta e mínimos locais. Apesar da proposta de outras abordagens para treinamento da rede, como, por exemplo, métodos de otimização de segunda ordem, subseleção de métodos ou métodos de otimização global, nenhum deles garante uma solução global ótima (BISHOP, 2006).

As ELMs foram desenvolvidas com o objetivo de manter as vantagens das redes MLP e reduzir os impactos de suas limitações. Esses modelos apresentam uma arquitetura de rede neural do tipo feedforward contendo uma camada escondida (SLFN) ${ }^{1}$ e que, em alguns estudos comparativos, mostram um desempenho superior, comparado às RNAs tradicionais (HUANG; ZHU; SIEW, 2006).

1 Do original, em inglês, Single-Hidden Layer Feedforward Neural Network. 
Figura 2 - Arquitetura de uma rede SLFN - Demonstração da estrutura de uma rede SLFN contendo uma camada de entrada com m nós, uma única camada oculta com n nós e uma camada de saída com m nós.

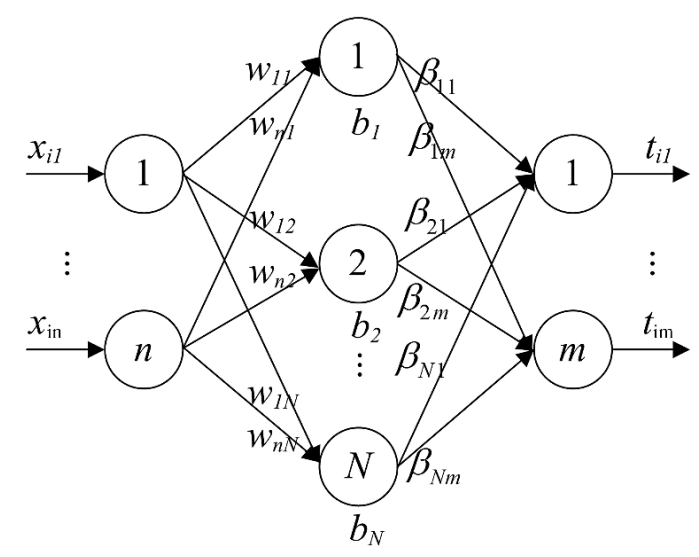

Fonte: Adaptado de Huang et al. (2015).

ELMs são uma alternativa ao uso de redes MLP, pois apesar de também possuírem um caráter conexionista, prometem vantagens, melhorando o tempo de treinamento e mantendo a flexibilidade no ajuste dos dados. Nesse tipo de modelo, os pesos das conexões que chegam à camada escondida são inicializados aleatoriamente com valores fixos, sem a necessidade de ajuste iterativo, reduzindo consideravelmente o tempo de treinamento da rede. Os únicos parâmetros que necessitam ser aprendidos são os pesos das conexões entre a camada oculta e a camada de saída, tornando o cálculo para aprendizagem do modelo um sistema linear a ser resolvido.

As ELMs se baseiam no princípio de que, com o número total de neurônios na camada oculta $(n h)$ é possível aprender sobre o mesmo número de eventos distintos (nt) com erro próximo de zero, adotando qualquer função de ativação não linear para as unidades de processamento (TERMENON et al., 2013). Além disso, o princípio da aproximação universal oferece embasamento para afirmar que existe um número finito de neurônios e certa configuração de pesos sinápticos que permitem obter um erro de aproximação relativamente baixo durante a fase de treinamento (M. Bazaraa, 2006).

As funções de ativação, neste caso, podem ser definidas aleatoriamente. Contudo, considerando $\mathbf{x}$ como o vetor de entrada da rede e a e b como pesos da camada escondida e bias, estudos apontam um melhor desempenho utilizando uma das funções listadas a seguir:

Função Sigmoidal

$$
G(\mathbf{a}, b, \mathbf{x})=\frac{1}{1+\exp ^{(-\mathbf{a} \cdot \mathbf{x}+b)}}
$$


Função Tangente Hiperbólica

$$
G(\mathbf{a}, b, \mathbf{x})=\frac{1+\exp ^{(-\mathbf{a} \cdot \mathbf{x}+b)}}{1+\exp ^{(-\mathbf{a x}+b)}} .
$$

Função Gaussiana

$$
G(\mathbf{a}, b, \mathbf{x})=\exp (-b|| \mathbf{x}-\mathbf{a} \|) .
$$

Função Multiquadrática

$$
G(\mathbf{a}, b, \mathbf{x})=\exp \left(\|\mathbf{x}-\mathbf{a}\|+b^{2}\right)^{\frac{1}{2}} .
$$

Função Degrau

$$
G(\mathbf{a}, b, \mathbf{x})=\left\{\begin{array}{l}
1, \text { se } \mathbf{a} \cdot \mathbf{x}-\mathrm{b} \geq 0 \\
0, \text { caso contrário }
\end{array}\right.
$$

Função Cosseno

$$
G(\mathbf{a}, b, \mathbf{x})=\cos (\mathbf{a} \cdot \mathbf{x}+b) .
$$

Diferente da necessidade de calcular todos os parâmetros em uma rede MLP, nas ELMs os ajustes se concentram no cálculo dos pesos das conexões que chegam à camada de saída utilizando os pesos das conexões que chegam à camada oculta e os dados de treinamento. Esse processo ajusta os pesos das conexões entre a camada escondida e a camada de saída de forma não iterativa por meio da minimização do erro de aproximação conforme a equação:

$$
\min _{\beta \in R^{L x m}}\|\boldsymbol{H} \beta-\boldsymbol{T}\|^{2}+\lambda\|\beta\|^{2} .
$$

Nesse processo, a matriz $\boldsymbol{H}$ corresponde à matriz de pesos da camada escondida, e $\boldsymbol{T}$ corresponde aos dados de treinamento. Para obtenção do parâmetro $\beta$ deve-se resolver a seguinte equação:

$$
\beta=\boldsymbol{H}^{\dagger} \boldsymbol{T}
$$

na qual $\boldsymbol{H}^{\dagger}$ corresponde à matriz generalizada inversa de Moore-Penrose (KATSIKIS; PAPPAS, 2008). Muitos métodos podem ser aplicados para calcular a matriz generalizada inversa $\boldsymbol{H}^{\dagger}$, incluindo métodos iterativos (PETKOVI, 2011), ortogonais (BARATA; 
HUSSEIN, 2012) e decomposição de valor singular (SVD)² (BARATA; HUSSEIN, 2012). Contudo, os métodos ortogonais e iterativos são evitados devido ao custo de processamento. O método SVD possibilita o cálculo da matriz $\boldsymbol{H}^{\dagger}$ da seguinte forma:

$$
\boldsymbol{H}^{\dagger}=\left(\boldsymbol{H}^{t} \boldsymbol{H}\right)^{-1} \boldsymbol{H}^{t}
$$

Neste caso, a obtenção dos pesos da camada escondida, considerando um fator de regularização $\lambda$, que oferece uma melhor estabilidade para o modelo, pode ser obtido a partir da resolução da seguinte equação:

$$
\beta=\boldsymbol{H}^{t}\left(\frac{\boldsymbol{I}}{\lambda}+\boldsymbol{H}^{t} \boldsymbol{H}\right)^{-1} \boldsymbol{H}^{t} \boldsymbol{T}
$$

Em contraste com as afirmações de que o modelo ELM apresenta erro próximo de zero em sua fase de treinamento (HUANG; ZHU; SIEW, 2006), estudos demonstram que em modelos SLFNs com uma determinada quantidade de nós na camada escondida, denotada por nh, função de ativação sigmoidal e escolha aleatória de pesos e bias, é possível aprender uma determinada quantidade de observações distintas $n t$ de forma exata, ou seja, com erro zero, nos casos em que o número de nós na camada escondida nh é maior ou igual ao número de elementos disponíveis para treinamento nt. Contudo, em casos nos quais erros são admitidos, permite-se a configuração de $n h$ menor que $n t$, alterando a forma de cálculo da matriz $\boldsymbol{H}^{\dagger}$ para a seguinte equação:

$$
\boldsymbol{H}^{\dagger}=\boldsymbol{H}^{t}\left(\boldsymbol{H} \boldsymbol{H}^{t}\right)^{-1}
$$

Nesse caso, a obtenção dos pesos da camada escondida, considerando um fator de regularização $\lambda$, pode ser obtido a partir da seguinte equação:

$$
\beta=\boldsymbol{H}^{t}\left(\frac{\mathbf{I}}{\lambda}+\mathbf{H H}^{t}\right)^{-1} \mathbf{T}
$$

Essa abordagem torna o processo de treinamento linear, minimizando os custos de processamento, sem perder desempenho em relação a outros modelos conexionistas tradicionais. Tais comparações foram descritas com aplicações em diferentes campos (HUANG; ZHU; SIEW, 2006; SMITH et al., 2011). É importante ressaltar a superioridade do desempenho das redes ELM também sobre o uso de outros modelos tradicionais, como SVMs e suas variantes (HUANG; ZHU; SIEW, 2006).

\footnotetext{
2 Do original, em inglês, Singular Value Decomposition.
} 


\subsection{Máquinas ELM para dados desbalanceados}

De acordo com o processo tradicional de classificação binária, os classificadores convencionais consideram o custo do erro de classificação das classes envolvidas como sendo iguais. Assim, durante o processo de treinamento, é recomendável utilizar qunatidades de exemplos aproximadamente iguais para as classes envolvidas. Entretanto, em problemas reais, nem sempre é possível a obtenção de conjuntos balanceados para a fase de treinamento. Para evitar que a presença demasiada de uma classe prejudique o desempenho do modelo ELM, é possível aplicar a técnica (W-ELM) ${ }^{3}$, uma generalização do ELM que recebe como entrada uma matriz $\mathbf{C}$ contendo os coeficientes de ponderação do erro de treinamento para cada uma das classes. Vale ressaltar que o custo do erro de classificação das classes envolvidas não está relacionado simplesmente a sua distribuição no conjunto de treinamento, mas também à importância intrínseca de cada classe no domínio do problema.

Sendo $\mathbf{C}$ uma matriz contendo os coeficientes de custo na diagonal e que é utilizada na obtenção de $\beta$, a inversabilidade matricial pode ser garantida da seguinte forma:

$$
f(x)= \begin{cases}\left(\boldsymbol{H}^{t} \boldsymbol{C H}+\frac{\boldsymbol{I}}{\lambda}\right)^{-1} \boldsymbol{H}^{t} \boldsymbol{C} \boldsymbol{T}, & \text { se nh }>=\text { nt, } \\ \boldsymbol{H}^{t}\left(\boldsymbol{C H} \boldsymbol{H}^{t}+\frac{\boldsymbol{I}}{\lambda}\right)^{-1} \boldsymbol{C} \boldsymbol{T}, & \text { caso contrário. }\end{cases}
$$

A matriz $\mathbf{C}$ possui os coeficientes que ponderam o erro de clasificação para cada uma das classes, apresentando um valor maior para as classes que possuem erros com menor custo e um valor menor para aquelas classes cujos erros têm custos mais significativos, reduzindo assim o risco de overfiting para a classe predominante.

\subsection{Discussão sobre o desempenho do modelo ELM}

Estudos preliminares apontam que o modelo ELM possui bons resultados na análise de conjuntos de dados conhecidos para a validação de classificadores (BLACKARD JOCK;J. DEAN, 1998) (HARRISON; RUBINFELD, 1978), atingindo marcas de desempenho superiores a outras técnicas, como SVM e ADAboost. Porém, com a finaldiade de avaliar o comportamento do modelo ELM na resolução de problemas mais complexos, o mesmo foi aplicado em tarefas de reconhecimento de imagens, mais especificamente no processo de reconhecimento facial e de micro expressões(LIU; WANG, 2010). No trabalho 
em questão, o modelo ELM juntamente com algoritmos de reconhecimento baseados em análise de tensor discriminante de sub-espaço (DTSA) ${ }^{4}$, obtendo resultados superiores a algoritmos tradicionais de reconhecimento, como, por exemplo, classificadores de vizinhos mais próximos $(\mathrm{K}-\mathrm{NN})^{5}$. Ainda no campo de reconhecimento de imagens, outro trabalho propôs o uso de comitês de ELM ${ }^{6}$ com seleção empírica de parâmetros para atingir melhores resultados(LIU; WANG, 2010). Testes experimentais monstraram que tal proposta, apesar de apresentar um sensível aumento no tempo de processamento, em relação ao ELM comum, atingiu $96.19 \%$ de acurácia, frente a algoritmos como K-NN e outras abordagens baseadas em retropropagação do erro consideradas no mesmo estudo. Outros trabalhos relevantes podem ser encontrados em uma coletânea publicada pelo laboratório de mídia do Instituto de Tecnologia de Massachusetts (MIT) (CAMBRIA et al., 2013), apontando pesquisas em diversas áreas assistidas por técnicas de aprendizagem de máquina.

\subsubsection{Críticas ao modelo ELM}

Apesar da existência de diversos trabalhos sobre a aplicação do modelo ELM pra solucionar vários problemas do mundo real e de um constante esforço de apresentar essa técnica como inovadora (HUANG, 2015), alguns membros da comunidade científica contestam as vantagens atribuídas à utilização dessa técnica. Um dos principais pontos de questionamento refere-se à arquitetura do modelo, uma vez que esta pode ser comparado a uma rede MLP de duas camadas, na qual a primeira camada possui seus pesos sinápticos fixos e definidos aleatoriamente, sendo seu treinamento concentrado apenas na segunda camada. Tal arquitetura assemelha-se aos modelos Gamba Perceptron (MINSKY MARVIN;PAPERT, 1971), Rosenblatt Perceptron e Redes de Base Radial (RBF) já conhecidos na literatura.

Além dessas semelhanças, em especial com relação à rede RBF, o modelo ELM também utiliza o cálculo da matriz pseudoinversa para a obtenção dos pesos das conexões entre a camada oculta e a camada de saída . Entretanto, o mesmo modelo não contempla algumas características que garantem o desempenho durante a resolução de problemas mais complexos com dados de difícil separação. Enquanto no modelo ELM os nós de

4 Do original, em inglês, Discriminant Tensor sub-space analysis

5 Do original, em inglês, K-Nearest Neighbors Classifiers

6 Ensemble-ELM 
processamento possuem as mesmas funções, o modelo RBF utiliza na camada de entrada um conjunto de funções capazes de efetuar transformações não-lineares do espaço de entrada, ao passo que a camada de saída é composta de nós que possuem funções de ativação lineares (LECUN'S, 2015).

Outro ponto de questionamento em relação ao modelo ELM deve-se ao fato de que a inicialização de sua camada oculta, feita de forma aleatória, não prioriza o uso de vetores de entrada dimensionalmente menores e nem contribui para tornar os dados mais facilmente separáveis, indo contra os avanços mais relevantes nessa área de pesquisa desde o surgimento dos primeiros perceptrons (DUDA et al., 2001). Ainda com relação à definição aleatória dos pesos, o modelo pode apresentar eventualmente bons resultados especificamente nos casos em que a função a ser aprendida é demasiadamente simples e o volume de dados de treinamento é reduzido, constituindo assim um problema de fácil regularização capaz ser resolvido eficientemente por SVM ou RBF, cenário este bem reduzido levando em consideração o vasto grupo de problemas mais complexos presentes na área de aprendizagem de máquina. 


\section{Avaliação de desempenho de classificadores}

Devido à grande variedade de técnicas e aplicações, diversos classificadores podem ser propostos para a solução de um memso problema. Até mesmo um único classificador possui uma série de generalizações que prometem solucionar problemas específicos, seja por detalhes de abordagens ou, em alguns casos, ajustes de parâmetros. Nesse sentido, é importante conhecer intrinsicamente o funcionamento do modelo utilizado para encontrar aquele que obtenha o melhor resultado na análise de novas instâncias (FERRI; HERNÁNDEZ-ORALLO; MODROIU, 2009). Com este fim, podem ser encontradas na literatura formas adequadas de se comparar classificadores utilizando algumas métricas de avaliação calculadas durante a fase de treinamento do mesmo.

\subsection{Métricas de Avaliação}

A maioria das abordagens para avaliação dos modelos preditivos deriva da análise da matriz de confusão. Muito utilizada na área da aprendizagem de máquina, essa matriz representa um esquema tabular de contigência que permite a visualização do desempenho de um modelo por meio de variáveis que refletem os resultados obtidos na fase de treinamento, levando em conta as classes preditas pelo modelo e as esperadas (FAWCETT, 2014), conforme apresentado na tabela 2 .

Tabela 2 - Matriz de Confusão

\begin{tabular}{|c|c|c|}
\hline & Positivo Esperado & Negativo Esperado \\
\hline Positivo Predito & Verdadeiro Positivo (TP) & Falso Positivo(FP) \\
\hline Negativo Predito & Falso Negativo(FN) & Verdadeiro Negativo(TN) \\
\hline
\end{tabular}

Na tabela 2, pode-se observar as seguintes variáveis: TP, que representa o número de verdadeiros positivos, ou seja, as instâncias com rotulação verdadeira identificadas corretamente, TN, número de instâncias negativas estimadas corretamente, FP, número de exemplos negativos, mas identificados como positivos e a variável FN representando o número de elementos positivos identificados erroneamente como negativos (FERRI; HERNÁNDEZ-ORALLO; MODROIU, 2009). De posse desses valores, uma série de métricas de avaliação podem ser calculadas, com a finalidade de estimar a eficiência do modelo de classificação. Dentre estas, destacam-se algumas mais comuns como: 
- Acurácia:

$$
\frac{T P+T N}{P+N}
$$

- Precisão:

$$
\frac{T P}{T P+F P}
$$

- Taxa de falsos positivos:

$$
t_{f p}=\frac{F P}{F P+T N}
$$

- Recall/Sensibilidade ou taxa de verdadeiros positivos:

$$
t_{v p}=\frac{T P}{T P+F N}
$$

- Especificidade ou taxa de verdadeiros negativos:

$$
t_{v n}=\frac{T N}{F P+T N}
$$

- F-score:

$$
F_{1}=\frac{\left(\beta^{2}+1\right) T P}{\left(\beta^{2}+1\right) T P+\beta^{2} F N+F P}
$$

Apesar do uso abrangente dessas métricas como avaliadores confiáveis, em alguns casos, em especial na presença de dados desbalanceados, algumas métricas, como precisão, não representam uma medida real do desempenho do modelo, uma vez que irão produzir indicadores errados (FAWCETT, 2014).

\subsection{Avaliação de dados desbalanceados}

Na presença de dados desbalanceados, o modelo de classifcação, caso não for adaptado, pode sofrer influência da classe majoritária e, assim, fornecer resultados enviesados e não reais, frente ao uso de métricas tradicionais de avaliação, como as citadas na seção anterior (BATISTA; PRATI; MONARD, 2004). Mais especificamente, medidas como acurácia consideram os custos de erro de classificação de cada classe iguais, o que não oferece maiores problemas no treinamento com dados balanceados. Mas nos casos em que a distribuição das classes é muito desigual, o classificador deve ser avaliado de forma específica.

Para cenários em que se observa desbalanceamento de classe, as taxas de falsos negativos e de falsos positivos podem ser consideradas como métricas de avaliação confiáveis 
(SOKOLOVA; LAPALME, 2009). Tais medidas podem servir como indicadores quantitativos do desempenho ou serem utilizados em demonstração gráfica por meio da análise de curvas das características operacionais do receptor (ROC) ${ }^{1}$.

\subsection{Análise gráfica de desempenho no espaço $R O C$}

Análise sob o espaço ROC consiste em um modelo de visualização de duas dimensões amplamente considerado na análise do desempenho de classificadores. Essa abordagem, empregada inicialmente em técnicas de detecção de sinais (EGAN, 1975), utiliza as taxas de verdadeiros positivos e falsos positivos para geração de um gráfico bidimensional que permite a análise de diversas características relacionadas ao modelo, entre estas, sua capacidade de classificar corretamente instâncias de forma aleatória (FAWCETT, 2014). Tal técnica foi aplicada com sucesso na análise de diversos problemas do mundo real, como comparação de algoritmos (PROVOST; FAWCETT; KOHAVI, 1997), sistemas de tomada de decisão médica (HAJIAN-TILAKI, 2013) e testes de diagnósticos (ZWEIG; CAMPBELL, 1993).

A análise baseada no espaço ROC não sofre o efeito da distribuição das classes (FAWCETT, 2014), sendo a forma de avaliação mais indicada para problemas nos quais diferentes custos de erro de classificação são considerados (SOKOLOVA; LAPALME, 2009). Contudo, vale ressaltar que apesar da aparente simplicidade, a técnica possui detalhes intrínsecos que devem ser cuidadosamente entendidos para permitir a correta análise resultados.

Para a análise de desempenho de um classificador discreto, em que uma matriz de confusão é obtida após sua etapa de treinamento, a avaliação do modelo produz um único ponto no espaço ROC. Neste caso, com base na matriz de confusão obtida pelo classificador, é calculado um único par de valores de taxa de verdadeiro positivo $t_{v p} \mathrm{e}$ taxa de falso positivo $t_{f p}$. Estes valores são então empregados para obtenção de um ponto $P R O C_{i}\left(t_{v p}, t_{f p}\right)$, posicionado no espaço ROC conforme ilustra a figura 3 :

\footnotetext{
1 Do original, em inglês, Receiver Operating Characteristic
} 
Figura 3 - Classificadores discretos (A,B,C,D e E) posicionados no espaço ROC. Para os classificadores localizados no triângulo superior esquerdo, quanto mais afastados os pontos da linha pontilhada, melhor o desempenho. Os classificadores localizados abaixo da linha pontilhada possuem desempenho menor que 50\%, considerando uma escolha aleatória de classe.

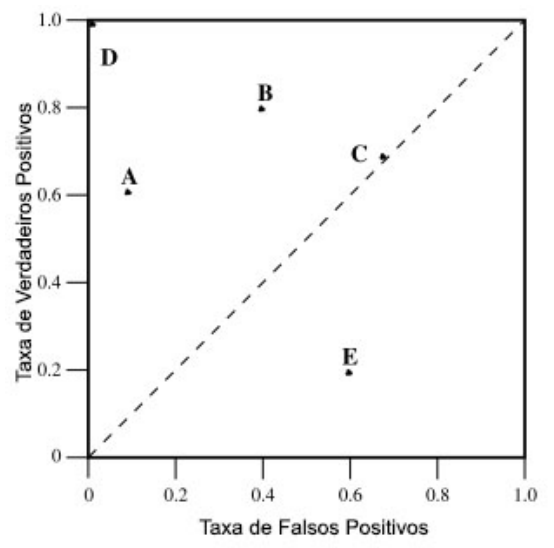

Fonte: Adaptado de Fawcett (2014).

A posição de um classificador no espaço ROC reflete caracteríticas relacionadas ao seu desempenho. Por exemplo, na figura 3, os classificadores que aparecem no do lado esquerdo próximos ao eixo $x,(\mathrm{~A}, \mathrm{~B}, \mathrm{C}$ e D), são considerados como classificadores conservadores. Nesse caso, a identificação das instâncias positivas é feita somente quando existe um alto grau de certeza, e poucos falsos positivos são encontrados (FAWCETT, 2014). De forma oposta, os classificadores posicionados no lado superior direito do espaço ROC são considerados como liberais, pois dependem de um nível baixo de certeza para classificação das instâncias positivas. Esse comportamento pode produzir altas taxas de falsos positivos(FAWCETT, 2014). Por questões de desempenho e confiança, é preferível que o classificador ideal se posicione na área esquerda do gráfico. 


\section{Resultados experimentais}

Conforme discutido anteriormente, a incerteza na rotulação dos dados de QM pode influenciar no desempenho dos classificadores baseados em aprendizagem de máquina. Com o objetivo de atenuar essa limitação, a presente pesquisa propôs um modelo capaz de tratar o problema da incerteza na rotulação de dados, possibilitando a construção de classificadores mais eficientes.

Este capítulo descreve o modelo proposto e discute resultados experimentais para dados de benchmark e conjuntos de dados de Química Medicinal.

\subsection{Modelo proposto}

O modelo proposto neste trabalho tem como objetivo final receber como entrada um conjunto de dados de QM, representando compostos químicos rotulados como ativos ou inativos; ou com rotulação inconclusiva. Dessa forma, utilizando o processo de aprendizado PU, as instâncias identificadas como negativas, ou inconclusivas têm seus rótulos estimados, com base na informação presente nos atributos dos próprios exemplos e nas características do conjunto de dados como um todo. Em seguida, os dados com seus novos rótulos são utilizados no treinamento de um classificador, que, por sua vez, será avaliado de acordo com métricas que possibilitem comparar o desempenho obtido com a nova rotulação estimada e com a rotulação original. De uma forma geral, a figura 4 apresenta os principais componentes do modelo. 
Figura 4 - Fluxo de tarefas do modelo prposto, que envolve tratamento dos dados, estimação de rótulos e classificação dos dados.

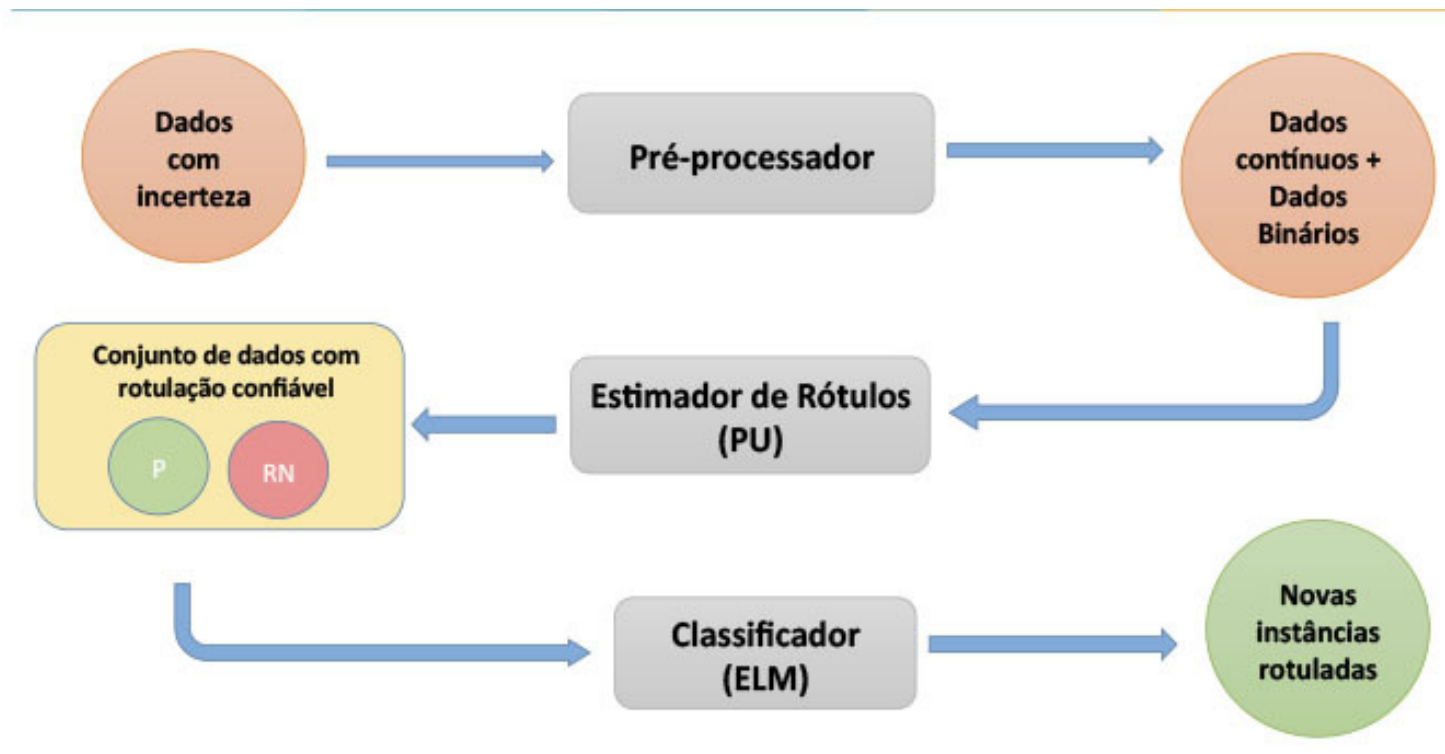

Fonte: SOUZA [2015].

O modelo aqui proposto pode ser dividido nas seguintes partes:

- Pré-processador: Durante a etapa de pré-processamento, os dados sob análise são submetidos a processos de padronização e discretização, com o intuito de adequar os dados para execução da técnica PU e do método ELM para classificação.

A padronização de dados consiste em redefinir cada dimensão dos dados, de modo que passe a apresentar média igual a zero e variância unitária. Na prática, calcula-se primeiro a média de cada dimensão (tomando-se os valores do conjunto de dados) e subtrai-se o resultado obtido dos valores em cada dimensão correspondente. Em seguida, cada dimensão é dividida pelo seu desvio padrão.

Métodos de discretização de dados são responsáveis por dividir a variação de valores de um atributo contínuo em intervalos, de modo a transformá-lo em um atributo discreto. Essa tarefa, quando executada em fases de pré-processamento, podem contribuir para agilizar a convergência de modelos iterativos (WIESLER; RICHARD; SCHL, 2014).

Devido à presença de incerteza na rotulação dos dados, impossibilitando o uso de técnicas de discretização supervisionadas, os seguintes métodos de discretização não supervisionados foram considerados no presente trabalho: 
- Número pré-determinado de intervalos uniformes $(E W)^{1}$ (DOUGHERTY; KOHAVI; SAHAMI, 1995): Esse método de discretização toma os valores mínimo (min), máximo $(\max )$ e o número total de instâncias $(r)$ para calcular um valor $t$, utilizado para definir o tamanho dos intervalos para um dado atributo, de acordo com a seguinte equação:

$$
t=(\max -\min ) / r .
$$

Em seguida, os intervalos podem ser obtidos com base em $t$ da seguinte forma:

$$
\min +t, \min +2 t, \ldots, \min +(k-1) t,
$$

em que $k$ é o número de intervalos.

- Número uniforme de amostras por intervalo (EF)² (JOIţA, 2010): Nessa abordagem, os dados são divididos em intervalos contendo aproximadamente a mesma quantidade de elementos. A quantidade de intervalos pode ser estimada utilizando $\sqrt{r}$, em que $r$ corresponde ao número total de instâncias.

Cabe ressaltar que, neste trabalho, serão exploradas algumas combinações das técnicas de pré-processamento mencionadas, com objetivo de avaliar quais destas produzem melhores resultados para o modelo aqui proposto. A escolha do método Naïve Bayes a ser considerado na estimação dos rótulos via PU dependerá da natureza dos dados, contínua (sem aplicar a discretização) ou discreta (aplicando a discretização). As duas variações da técnica NB podem ser vistas no capítulo 3.

- Rotulador: Baseado no aprendizado PU, este componente tem a função de produzir os subconjuntos requeridos pelo método, efetuar a seleção aleatória das instâncias que serão utilizadas como espiãs e executar o processo de estimação EM para obtenção das novas rotulações.

- Classificador: Utiliza a rotulação estimadas do conjunto de treinamento para criar o classificador baseado em ELM.

Vale ainda ressaltar que esses componentes foram executados em um computador contendo processador Intel ${ }^{\circledR}$ Core $^{\mathrm{TM}} \mathrm{i} 7-2700 \mathrm{~K}$ CPU $3.50 \mathrm{GHz} 8$, placa gráfica Gallium 0.4 on NVCE, disco rígido de 967,5 GB e sistema Ubuntu 15.1064 bits.

1 Do original, em inglês, Equal-interval binning.

2 Do original, em inglês, Equal-frequency binning. 
Para avaliar o desempenho da rotulação via PU, inicialmente considerou-se o conjunto de dados Iris, bem conhecido na área de aprendizagem de máquina. Tais dados serão brevemente descritos a seguir, juntamente com o processo para adequá-los ao problema de incerteza na rotulação aqui considerado. Na sequência, algumas discussões sobre os resultados dos experimentos para esses dados são apresentadas.

\subsection{Experimentos Preliminares}

\subsubsection{Descrição dos dados}

O conjunto de dados Iris (DUDA; HART; STORK, 2000) contém três classes que representam tipos de flores (setosa, versicolor e virginica), com 50 exemplos em cada uma, e quatro atributos numéricos contínuos. Uma das classes (setosa) é linearmente separável das outras duas (versicolor e virginica), as quais não são linearmente separáveis entre si. Para maiores detalhes, esse conjunto de dados encontra-se disponível no repositório UCI (LICHMAN, 2013).

\subsubsection{Adequação dos dados ao problema}

De modo a adequar o conjunto de dados Iris aos experimentos deste trabalho, inicialmente considerou-se como elementos positivos os exemplos da classe setosa e como negativos os exemplos pertencentes às classes versicolor e virginica. Em seguida, procedeuse com a alteração aleatória de $30 \%$ dos rótulos dos elementos positivos para negativos, de modo a simular a incerteza na rotulação.

A distribuição de classes do conjunto de dados, antes e após a mudança nos rótulos, pode ser observada na tabela 3. Na figura 6, observa-se, ainda, a projeção via PCA para os dados originais (9a) e para os dados após a adequação ao problema (9b).

Tabela 3 - Distribuição de classes do conjunto Iris antes e após alteração da rotulação.

\begin{tabular}{|c|c|}
\hline & (\#Positivos ; \#Negativos) \\
\hline Rotulação Original & $(50 ; 100)$ \\
\hline Rotulação após alteração de rótulos & $(35 ; 115)$ \\
\hline
\end{tabular}




\subsubsection{Experimentos de rotulação via PU}

Os conjuntos de dados obtidos durante a fase de pré-processamento foram submetidos ao rotulador PU na expectativa de que as instâncias positivas que obtiveram sua rotulação alterada reassumissem a rotulação original após o processo de estimação.

Além do tratamento da incerteza, também considerou-se os impactos causados pelo desbalanceamento das classes, adicionando ao algoritmo PU fatores de custo para compensar o viés causado pela classe majoritária. Não foi utilizada nenhuma heurística para estimar o fator de custo mais adequado para cada conjunto de dados, contudo, os seguintes custos foram considerados:

$$
\begin{gathered}
\frac{1}{\text { total }_{p}+\text { total }_{n}}, \\
0.5+\frac{\text { total }_{p}}{\text { total }_{n}}, \\
1.0, \\
1.0+\frac{\text { total }_{p}}{\text { total }_{n}},
\end{gathered}
$$

em que total $_{p}$ e total $_{n}$ correspondem, respectivamente, ao total de elementos positivos e total de elementos negativos no conjunto de dados.

Os resultados obtidos pelo rotulador PU, considerando diferentes versões préprocessadas do conjunto de dados e diferentes fatores de custo, podem ser observados na tabela 4 .

Tabela 4 - Distribuições de classes obtidas após aplicação do algoritmo PU ao conjunto de dados Iris com 30\% dos exemplos da classe positiva modificados para classe negativa. Contempla-se, ainda, a aplicação de técnicas de discretização como Equal Width (EW) e Equal Frequency (EF) e padronização na fase de pré-processamento dos dados.

\begin{tabular}{|c|c|c|c|c|}
\hline Fator de custo & $\frac{1}{\text { total }_{p}+\text { total }_{n}}$ & $0.5+\frac{\text { total }_{p}}{\text { total }_{n}}$ & 1.0 & $1.0+\frac{\text { total }_{p}}{\text { total }_{n}}$ \\
\hline Conjunto de dados & $(37 ; 113)$ & $(35 ; 115)$ & $(35 ; 115)$ & $(39 ; 111)$ \\
\hline Dados Originais & $(35 ; 115)$ & $(35 ; 115)$ & $(35 ; 115)$ & $(38 ; 112)$ \\
\hline Dados Originais + EF & $(36 ; 114)$ & $(36 ; 114)$ & $(36 ; 114)$ & $(36 ; 114)$ \\
\hline Dados Originais + Padronização + EF & $(39 ; 111)$ & $(46 ; 104)$ & $(39 ; 111)$ & $(39 ; 111)$ \\
\hline Dados Originais + EW & $(39 ; 111)$ & $(40 ; 110)$ & $(48 ; 102)$ & $(39 ; 111)$ \\
\hline
\end{tabular}


Em relação à tabela 4, é possível observar que, após o processo PU, a rotulação dos dados foi alterada na maioria dos casos. Entretanto, observa-se que, para os fatores de custo $0.5+\frac{t_{\text {total }}}{\text { total }_{n}}$ e 1.0, a etapa de estimação de rótulos identificou de 11 a 13 das 15 instâncias positivas modificadas inicialmente para negativas. Nota-se que a alteração mais significativa refere-se ao experimento para dados pré-processados com padronização e discretização EW e fator de custo igual a 1.0. Esse resultado pode ser visualizado na figura $6 \mathrm{~b}$.

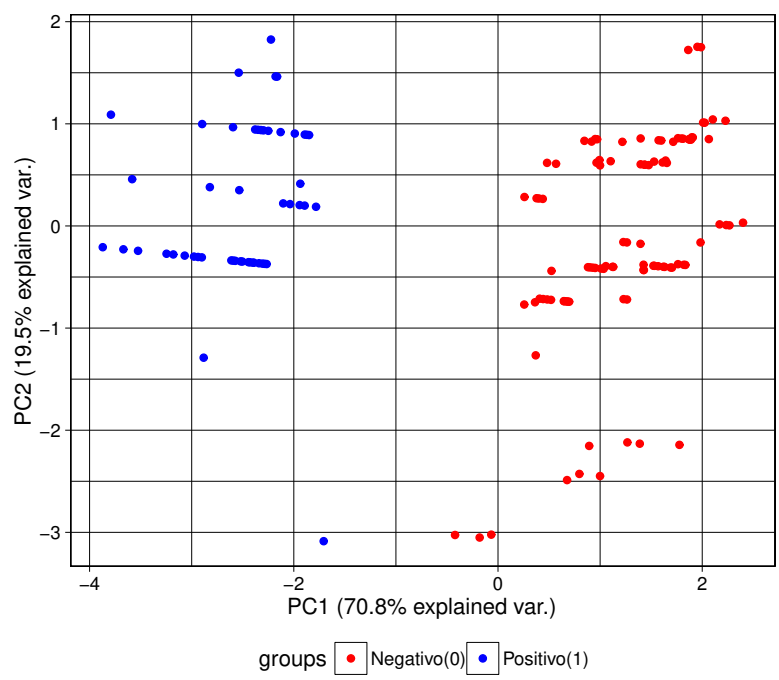

(a)

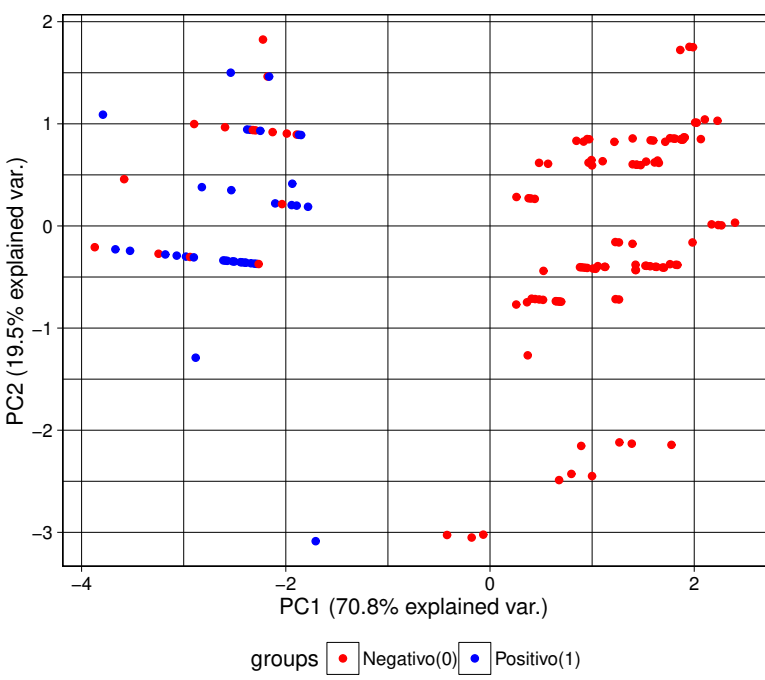

(b)

Figura 5 - (a) Projeção via PCA conjunto de dados Iris com os elementos da classe setosa definidas como positivas e os demais elementos versicolor e virgínica definidos como negativos. (b) Projeção via PCA do conjunto de dados Iris após modificação de $30 \%$ dos rótulos positivos. 


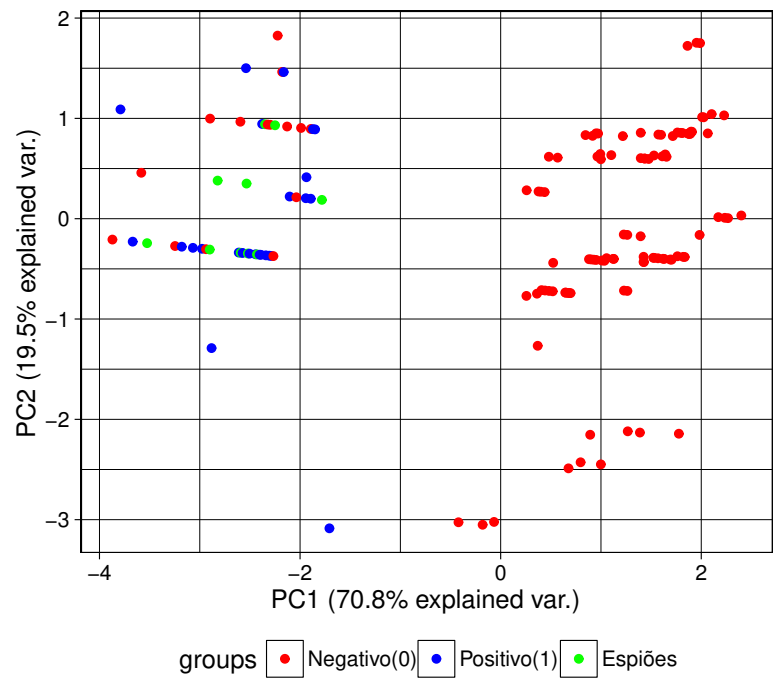

(a)

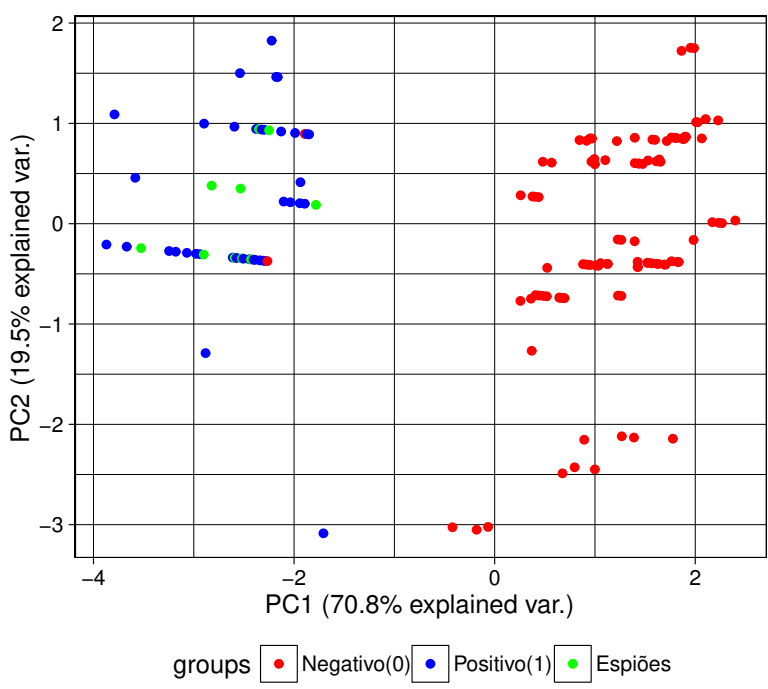

(b)

Figura 6 - (a) Rotulação inicial contendo as intâncias positivas com 30\% dos elementos alterados e $10 \%$ dos objetos positivos utilizados como espiões. (b) Rotulação estimada pelo método PU.

Como pode-se observar, o algoritmo de rotulação PU foi capaz de revelar a maioria das instâncias positivas, alteradas propositalmente.

Nos próximos experimentos, classificadores ELM serão construídos para o conjunto de dados Iris, levando em conta sua rotulação original, a rotulação alterada de forma aleatória e as rotulações estimadas pelo método PU. Objetiva-se, com essa análise, avaliar o quanto esses diferentes cenários afetam o desempenho final do modelo de classificação.

\subsubsection{Experimentos de classificação via ELM}

Cenário 1: Dados com rotulação original

Considerando os rótulos originais do conjunto Iris, essa seção apresenta os resultados obtidos pelo classificador ELM, variando o número de neurônios do modelo (500, 1000 e 1500) e as funções de ativação (Linear, Sigmoidal e RBF). O processo de validação cruzada foi aplicado, considerando 3 folds e os melhores resultados obtidos são apresentados na tabela 5 . 
Tabela 5 - Melhores resultados obtidos pelo classificador ELM para os dados originais, considerando variações do número de neurônios e funções de ativação. Resultados ordenados pela métrica $f$-score.

\begin{tabular}{|c|c|c|c|c|c|c|c|c|c|c|c|c|c|}
\hline id & VP & FP & VN & FN & Pre. & Rec. & $t_{f p}$ & $t_{v p}$ & Acc & $f_{1}$ & Sca. & Neu. & Fa. \\
\hline c0 & 49 & 0 & 100 & 1 & 0.98 & 0.98 & 0.0 & 0.98 & 0.99 & 0.49 & - & 1000 & sigm \\
c1 & 48 & 0 & 100 & 2 & 0.96 & 0.96 & 0.0 & 0.96 & 0.99 & 0.49 & - & 500 & sigm \\
c2 & 48 & 0 & 100 & 2 & 0.96 & 0.96 & 0.0 & 0.96 & 0.99 & 0.49 & - & 1500 & sigm \\
c3 & 48 & 0 & 100 & 2 & 0.96 & 0.96 & 0.0 & 0.96 & 0.99 & 0.49 & $\mathrm{~S}$ & 1500 & sigm \\
c4 & 48 & 0 & 100 & 2 & 0.96 & 0.96 & 0.0 & 0.96 & 0.99 & 0.49 & $\mathrm{~S}$ & 1000 & sigm \\
c5 & 47 & 0 & 100 & 3 & 0.94 & 0.94 & 0.0 & 0.94 & 0.98 & 0.48 & $\mathrm{~S}$ & 500 & $\mathrm{rbf}$ \\
c6 & 47 & 0 & 100 & 3 & 0.94 & 0.94 & 0.0 & 0.94 & 0.98 & 0.48 & $\mathrm{~S}$ & 500 & sigm \\
c7 & 45 & 1 & 99 & 5 & 0.9 & 0.9 & 0.01 & 0.9 & 0.96 & 0.47 & $\mathrm{~S}$ & 1500 & $\mathrm{rbf}$ \\
$\mathrm{c} 8$ & 45 & 0 & 100 & 5 & 0.9 & 0.9 & 0.0 & 0.9 & 0.97 & 0.47 & - & 500 & $\mathrm{rbf}$ \\
$\mathrm{c} 9$ & 45 & 0 & 100 & 5 & 0.9 & 0.9 & 0.0 & 0.9 & 0.97 & 0.47 & $\mathrm{~S}$ & 1000 & $\mathrm{rbf}$ \\
$\mathrm{c} 10$ & 38 & 0 & 100 & 12 & 0.76 & 0.76 & 0.0 & 0.76 & 0.92 & 0.43 & - & 1500 & $\mathrm{rbf}$ \\
\hline
\end{tabular}

O melhor resultado obtido nesses experimentos, considerando a métrica acurácia, foi de 99\%, para um modelo com 1000 neurônios e função sigmoidal. Cabe ressaltar que, para esses experimentos, bons resultados já eram esperados, dado que o conjunto de dados em questão é linearmente separável.

Cenário 2: Dados com rotulação alterada

Os experimentos descritos nesta seção destinam-se a avaliar o comportamento do modelo de classificação frente a cenários de rotulação incerta. Nesse sentido, um classificador ELM será construído para o conjunto de dados Iris com 30\% das instâncias positivas alteradas propositalmente para negativas, com a finalidade de simular dados com incerteza na rotulação.

A tabela 6 exibe os melhores resultados do classificador ELM para os dados alterados, sem a aplicação do estimador PU. Nota-se que, nesse caso, o classificador obteve desempenho inferior àquele atingido para os dados originais, apresentando no máximo 89\% de acurácia, e produzindo um alto número de elementos classificados como positivos incorretamente, independente das diversas variações de parâmetros aplicadas ao modelo. 
Tabela 6 - Melhores resultados obtidos pelo classificador ELM para os dados com rotulação alterada, considerando variações do número de neurônios e funções de ativação. Resultados ordenados pela métrica $f$-score.

\begin{tabular}{|c|c|c|c|c|c|c|c|c|c|c|c|c|c|c|}
\hline id & VP & FP & VN & FN & Pre. & Rec. & $t_{f p}$ & $t_{v p}$ & Acc & $f_{1}$ & Sca. & Neu. & Fa. & \\
\hline c0 & 33 & 15 & 100 & 2 & 0.69 & 0.94 & 0.13 & 0.94 & 0.89 & 0.80 & $\mathrm{~S}$ & EW & 1500 & sig \\
c1 & 26 & 7 & 108 & 9 & 0.79 & 0.74 & 0.06 & 0.74 & 0.89 & 0.76 & $\mathrm{~S}$ & EF & 500 & sig \\
c2 & 30 & 18 & 97 & 5 & 0.63 & 0.86 & 0.16 & 0.86 & 0.85 & 0.72 & - & - & 1500 & sig \\
c3 & 26 & 12 & 103 & 9 & 0.68 & 0.74 & 0.1 & 0.74 & 0.86 & 0.71 & $\mathrm{~S}$ & EF & 1000 & sig \\
c4 & 25 & 12 & 103 & 10 & 0.68 & 0.71 & 0.1 & 0.71 & 0.85 & 0.69 & $\mathrm{~S}$ & EF & 1500 & sig \\
c5 & 22 & 8 & 107 & 13 & 0.73 & 0.63 & 0.07 & 0.63 & 0.86 & 0.68 & $\mathrm{~S}$ & EW & 1000 & rbf \\
c6 & 22 & 8 & 107 & 13 & 0.73 & 0.63 & 0.07 & 0.63 & 0.86 & 0.68 & $\mathrm{~S}$ & EW & 1500 & sig \\
c7 & 22 & 8 & 107 & 13 & 0.73 & 0.63 & 0.07 & 0.63 & 0.86 & 0.68 & - & EW & 1500 & rbf \\
c8 & 22 & 9 & 106 & 13 & 0.71 & 0.63 & 0.08 & 0.63 & 0.85 & 0.67 & S & EW & 500 & sig \\
c9 & 22 & 9 & 106 & 13 & 0.71 & 0.63 & 0.08 & 0.63 & 0.85 & 0.67 & - & EW & 1000 & rbf \\
\hline
\end{tabular}

Cenário 3: Dados com rotulação estimada via PU

Após aplicar o algoritmo PU aos dados Iris com rotulação alterada, avaliou-se os como os novos rótulos estimados podem influenciar o desempenho do classificador ELM. Na tabela 7, pode-se observar os melhores resultados de classificação obtidos nesses experimentos.

Tabela 7 - Melhores resultados obtidos pelo classificador ELM para os dados com rotulação alterada, considerando variações do número de neurônios e funções de ativação. Resultados ordenados pela métrica $f$-score.

\begin{tabular}{|c|c|c|c|c|c|c|c|c|c|c|c|c|c|c|}
\hline id & VP & FP & VN & FN & Pre. & Rec. & $t_{f p}$ & $t_{v p}$ & Acc & $f_{1}$ & Sca. & Neu. & Fa. & \\
\hline c0 & 31 & 6 & 109 & 4 & 0.84 & 0.89 & 0.05 & 0.89 & 0.93 & 0.86 & - & EF & 500 & sig \\
c1 & 32 & 8 & 107 & 3 & 0.8 & 0.91 & 0.07 & 0.91 & 0.93 & 0.85 & - & EF & 1000 & sig \\
c2 & 29 & 6 & 109 & 6 & 0.83 & 0.83 & 0.05 & 0.83 & 0.92 & 0.83 & - & EF & 1500 & rbf \\
c3 & 32 & 10 & 105 & 3 & 0.76 & 0.91 & 0.09 & 0.91 & 0.91 & 0.83 & - & EF & 1500 & sig \\
c4 & 30 & 9 & 106 & 5 & 0.77 & 0.86 & 0.08 & 0.86 & 0.91 & 0.81 & - & EF & 1000 & rbf \\
c5 & 33 & 15 & 100 & 2 & 0.69 & 0.94 & 0.13 & 0.94 & 0.89 & 0.8 & - & - & 1500 & sig \\
c6 & 32 & 15 & 100 & 3 & 0.68 & 0.91 & 0.13 & 0.91 & 0.88 & 0.78 & - & - & 1000 & sig \\
c7 & 29 & 12 & 103 & 6 & 0.71 & 0.83 & 0.1 & 0.83 & 0.88 & 0.76 & S & EW & 1500 & sig \\
c8 & 28 & 12 & 103 & 7 & 0.7 & 0.8 & 0.1 & 0.8 & 0.87 & 0.75 & S & EW & 1000 & sig \\
c9 & 28 & 13 & 102 & 7 & 0.68 & 0.8 & 0.11 & 0.8 & 0.87 & 0.74 & - & - & 500 & rbf \\
\hline
\end{tabular}

Nesse caso, nota-se que o algoritmo PU impactou de forma positiva o processo de classificação, que chegou a atingir 93\% de acurácia, superando a taxa máxima de $89 \%$ obtida para o conjunto de dados com rotulação alterada. 
De forma geral, considerando todos os resultados obtidos para o conjunto Iris, foi possível observar que o processo de estimação de rótulos mais confiáveis favorece o classificador final, nesse caso, o modelo baseado em ELM. Além da redução na quantidade de falso positivos e falsos negativos, a acurácia foi de 93\%, maior que o erro majoritário de 66,6\% e superior à taxa máxima de $89 \%$ obtida para os dados com rotulação alterada. Além disso, o melhor resultado foi atingido com somente 500 neurônios na camada escondida, a menor quantidade utilizada nesse experimento.

Dados os resultados obtidos durante os experimentos preliminares, espera-se que o rotulador PU contribua positivamente para os resultados do classificador ELM aplicado a dados de natureza mais complexa. Na sessão seguinte, serão discutidos resultados experimentais para dados específicos de Química Medicinal.

\subsection{Experimentos com dados de Química Medicinal}

Nos experimentos descritos nessa seção, conjuntos de dados específicos de Química Medicinal foram submetidos ao modelo proposto neste trabalho, com o principal objetivo de avaliar o desempenho da abordagem no contexto de QM.

\subsubsection{Descrição dos dados}

Para os experimentos deste trabalho, foram selecionados cinco conjuntos de dados contendo informações sobre compostos bioativos e atividade biológica relacionadas ao estudo de morte celular, regulação de contração muscular e estudos sobre alzheimer. Descrições breves para cada um desses conjuntos são dadas a seguir.

AID1608 - Consiste em um ensaio realizado com o objetivo de identificar compostos capazes de reduzir a toxicidade responsável por morte celular, causada pela presença da poliglutamina HttQ103 induzida. As células analisadas foram monitoradas em relação à liberação do Ácido Lático Dehidrogenase, sendo que os compostos que apresentaram manifestação ao descritor httLDHEC_50 foram identificados como ativos e os elementos restantes foram rotulados como apresentando atividade inconclusiva (PUBCHEM, 2016a). O conjunto de dados AID1608 é composto por 1.033 compostos, com a proporção de um composto ativo para 14 compostos inconclusivos, apresentando, portando a distribuição de 
6,58\% elementos da classe minoritária. Ainda, neste conjunto de dados, é possível encontrar 30 características contendo valores contínuos e 882 características que apresentam valores categóricos.

AID644 - Refere-se a um bioensaio realizado com o objetivo de identificar elementos capazes de inibir a Rho-quinase, uma enzima envolvida na regulação da contração do músculo liso e reorganização do citoesqueleto de células não musculares.Neste conjunto de dados, é possível encontrar 27 características contendo valores contínuos e 74 características que apresentam valores categóricos. Os inibidores de moléculas pequenas da Rho-quinase, que promovem o relaxamento do músculo liso, podem ser eficazes no tratamento do vasoespasmo cerebral e potencialmente eficazes no tratamento da angina, hipertensãO, arteriosclerose e disfunção erétil (PUBCHEM, 2016b).

AID1284 - É um conjunto de dados direcionado à identificação de JNK3, uma enzima ativada especificamente no cérebro, que possui relação comprovada com a morte de células dopaminérgicas, possuindo, assim, um papel fundamental no estudo sobre alzheimer (PUBCHEM, 2016c). É possível encontrar, neste conjunto de dados, 27 características contendo valores contínuos e 77 características que apresentam valores categóricos.

AID721 - Consiste em um ensaio relacionado à identificação de compostos ativadores de Proteíno-quinases estimuladas por mitógeno, um tipo particular de proteína responsável pela regulação de atividades celulares como mitose, sobrevivência e morte celular (PUBCHEM, 2016d). O conjunto de dados em questão apresenta 28 características contendo valores contínuos e 60 características que apresentam valores categóricos.

AID439 - Representa substâncias testadas para Esfingosina 1-fosfato, um receptor relacionado à regulação de funções celulares como proliferação, diferenciação e migração celular (PUBCHEM, 2016e). Apresenta 28 características contendo valores contínuos e 54 características contendo valores categóricos.

A tabela 1 apresenta algumas informações sobre os conjuntos de QM aqui considerados, tal como número de instâncias, proporção de elementos da classe minoritária (compostos ativos). 
Tabela 8 - Informações resumidas sobre os conjuntos de dados de QM analisados.

\begin{tabular}{|c|c|c|}
\hline Dados & Observações & $\%$ Ativos \\
\hline AID1608 & 1033 & $6.58 \%$ \\
AID644 & 206 & $32.52 \%$ \\
AID1284 & 362 & $15.75 \%$ \\
AID721 & 94 & $22.34 \%$ \\
AID439 & 69 & $18.84 \%$ \\
\hline
\end{tabular}

Nas próximas seções, serão discutidos os resultados experimentais para os conjuntos de QM mencionados, em que serão avaliados o algoritmo PU adaptado ao tratamento do desbalanceamento de classes e o impacto de seus resultados no desempenho de classificadores ELM, com a mesma variação de parâmetros adotada na execução dos experimentos preliminares.

\subsubsection{Experimentos de rotulação via PU - Conjunto de dados AID1284}

Inicialmente, o conjunto de dados AID1284 foi submetido à etapa de rotulação PU. Para isso, foram consideradas diversas versões pré-processadas do mesmo conjunto, com o objetivo de avaliar como os métodos de discretização e padronização afetam a obtenção das novas rotulações. Além disso, para tratar o desbalanceamento dos dados, a aplicação de fatores de custos diferentes também foi explorada. Considerando a distribuição de classes inicial, com 57 elementos positivos e 305 negativos, as novas distribuições obtidas após aplicação da técnica PU são apresentadas na tabela 9. 
Tabela 9 - Distribuições de classes do conjunto de dados AID1284 obtidas após a execução do processo PU, com variação de fatores de custo.

\begin{tabular}{|c|c|c|c|c|}
\hline Conjunto de dados Custo & $\frac{1}{\text { total }_{p}+\text { total }_{n}}$ & $0.5+\frac{\text { total }_{p}}{\text { total }_{n}}$ & 1.0 & $1.0+\frac{\text { total }_{p}}{\text { total }_{n}}$ \\
\hline Bin. e Cont. + EW & $355 / 7$ & $58 / 304$ & $69 / 293$ & $60 / 302$ \\
\hline Cont. + Padronização & $57 / 305$ & $92 / 270$ & $59 / 303$ & $62 / 300$ \\
\hline Bin. & $353 / 9$ & $91 / 271$ & $79 / 283$ & $60 / 302$ \\
\hline Bin. e Cont. & $57 / 305$ & $60 / 302$ & $60 / 302$ & $62 / 300$ \\
\hline Cont. & $57 / 305$ & $102 / 260$ & $58 / 304$ & $58 / 304$ \\
\hline Bin. e Cont. + Scale & $57 / 305$ & $60 / 302$ & $60 / 302$ & $62 / 300$ \\
\hline Cont. + Padronização & $57 / 305$ & $102 / 260$ & $58 / 304$ & $58 / 304$ \\
\hline Bin. e Cont. + EF & $290 / 72$ & $58 / 304$ & $59 / 303$ & $58 / 304$ \\
\hline Bin. e Cont. + Padronização + EF & $311 / 51$ & $58 / 304$ & $62 / 300$ & $63 / 299$ \\
\hline Cont. + EF & $57 / 305$ & $63 / 299$ & $59 / 303$ & $72 / 290$ \\
\hline Cont. + Scale + EW & $253 / 109$ & $59 / 303$ & $128 / 234$ & $85 / 277$ \\
\hline Cont. + Scale + EF & $202 / 160$ & $69 / 293$ & $60 / 302$ & $81 / 281$ \\
\hline Bin. e Cont. + Padronização + EW & $351 / 11$ & $62 / 300$ & $60 / 302$ & $60 / 302$ \\
\hline Cont. + EW & $313 / 49$ & $69 / 293$ & $96 / 266$ & $85 / 277$ \\
\hline
\end{tabular}

Com base nos resultados obtidos, observa-se que quando considerados apenas as características binárias dos dados e o menor custo, o estimador PU altera a maior parte dos elementos negativos para positivos. Por outro lado, o mesmo não ocorre quando somente características contínuas são consideradas. É possível observar, ainda, que os resultados mais estáveis do modelo não dependem somente dos custos utilizados, mas também dos métodos utilizados no pré processamento.

\subsubsection{Experimentos de classificação via ELM - Conjunto de dados AID1284}

Nos próximos experimentos, classificadores ELM serão construídos para o conjunto de dados AID1284, levando em conta sua rotulação original e as rotulações estimadas pelo método PU. Objetiva-se, com essa análise, avaliar o quanto esses diferentes cenários afetam o desempenho final do modelo de classificação.

Cenário 1: Análise de classificadores ELM com fator de custo $C_{i j}=\frac{1}{\text { total }_{p}+\text { total }_{n}}$

Esta seção apresenta uma análise comparativa entre os resultados obtidos pelo classificador ELM com fator de custo $C_{i j}=\frac{1}{\text { total }_{p}+\text { total }_{n}}$, considerando a rotulação original do conjunto AID1284 e a rotulação obtida após aplicação a técnica PU. 
Os resultados alcançados pelo classificador construído para os dados com rotulação original são apresentados na tabela 10. O melhor resultado foi obtido para os dados padronizados e com discretização EF e para o modelo ELM com 1500 neurônios e função de ativação sigmoidal. Nota-se, entretanto, que esse desempenho está bem próximo do erro majoritário para o conjunto, que é igual a $84,25 \%$ e, portanto, considerado insatisfatório.

Tabela 10 - Resultados obtidos pelo classificador ELM com fator de custo $C_{i j}=\frac{1}{\text { total }_{p}+\text { total }_{n}}$ para o conjunto de dados AID1284 com rotulação original.

\begin{tabular}{|c|c|c|c|c|c|c|c|c|c|c|c|c|c|c|}
\hline id & VP & FP & VN & FN & Pre. & Rec. & $t_{f p}$ & $t_{v p}$ & Acc & $f_{1}$ & Scale & Disc & Neu. & Fa. \\
\hline c0 & 32 & 34 & 271 & 25 & 0.48 & 0.56 & 0.11 & 0.56 & 0.84 & 0.52 & $\mathrm{~S}$ & $\mathrm{EF}$ & 1500 & $\mathrm{sig}$ \\
c1 & 21 & 13 & 292 & 36 & 0.62 & 0.37 & 0.04 & 0.37 & 0.86 & 0.46 & $\mathrm{~S}$ & $\mathrm{EF}$ & 500 & $\mathrm{rbf}$ \\
c2 & 16 & 5 & 300 & 41 & 0.76 & 0.28 & 0.02 & 0.28 & 0.87 & 0.41 & $\mathrm{~S}$ & $\mathrm{EF}$ & 1000 & $\mathrm{rbf}$ \\
c3 & 22 & 33 & 272 & 35 & 0.4 & 0.39 & 0.11 & 0.39 & 0.81 & 0.39 & $\mathrm{~S}$ & $\mathrm{EF}$ & 1500 & $\mathrm{rbf}$ \\
c4 & 23 & 38 & 267 & 34 & 0.38 & 0.4 & 0.12 & 0.4 & 0.8 & 0.39 & $\mathrm{~S}$ & $\mathrm{EF}$ & 1000 & $\mathrm{sig}$ \\
c5 & 19 & 50 & 255 & 38 & 0.28 & 0.33 & 0.16 & 0.33 & 0.76 & 0.3 & $\mathrm{~S}$ & $\mathrm{EF}$ & 500 & $\mathrm{sig}$ \\
c6 & 44 & 204 & 101 & 13 & 0.18 & 0.77 & 0.67 & 0.77 & 0.4 & 0.29 & $\mathrm{~S}$ & - & 500 & lin \\
c7 & 44 & 205 & 100 & 13 & 0.18 & 0.77 & 0.67 & 0.77 & 0.4 & 0.29 & $\mathrm{~S}$ & - & 1500 & rbf \\
c8 & 44 & 205 & 100 & 13 & 0.18 & 0.77 & 0.67 & 0.77 & 0.4 & 0.29 & $\mathrm{~S}$ & - & 1500 & rbf \\
c9 & 36 & 157 & 148 & 21 & 0.19 & 0.63 & 0.51 & 0.63 & 0.51 & 0.29 & - & $\mathrm{EW}$ & 1000 & sig \\
\hline
\end{tabular}

Após a aplicação da técnica PU, novos classificadores foram construídos e seus resultados (ver tabela 11) foram analisados. Observa-se, agora, que o modelo ELM atingiu a acurácia de $95 \%$, levando em consideração a utilização da técnica de discretização EF, 1500 neurônios na camada escondida e função de ativação sigmoidal, o que representa uma melhora significativa de desempenho em comparação aos resultados com rotulação original. Outros destaques destes experimentos são a inexistência de falsos positivos e a diminuição no número de falsos negativos, de 25 para 17.

Tabela 11 - Resultados obtidos pelo classificador ELM com fator de custo $C_{i j}=\frac{1}{\text { total }_{p}+\text { total }_{n}}$ para o conjunto de dados AID1284 após a aplicação do rotulador PU.

\begin{tabular}{|c|c|c|c|c|c|c|c|c|c|c|c|c|c|c|}
\hline id & VP & FP & VN & FN & Pre. & Rec. & $t_{f p}$ & $t_{v p}$ & Acc & $f_{1}$ & Scale & Disc & Neu. & Fa. \\
c0 & 40 & 0 & 305 & 17 & 1.0 & 0.7 & 0.0 & 0.7 & 0.95 & 0.82 & - & EF & 1500 & sig \\
c1 & 40 & 0 & 305 & 17 & 1.0 & 0.7 & 0.0 & 0.7 & 0.95 & 0.82 & - & EF & 1500 & sig \\
c2 & 39 & 6 & 299 & 18 & 0.87 & 0.68 & 0.02 & 0.68 & 0.93 & 0.76 & $\mathrm{~S}$ & EF & 1500 & sig \\
c3 & 37 & 10 & 295 & 20 & 0.79 & 0.65 & 0.03 & 0.65 & 0.92 & 0.71 & $\mathrm{~S}$ & $\mathrm{EF}$ & 1000 & sig \\
c4 & 29 & 8 & 297 & 28 & 0.78 & 0.51 & 0.03 & 0.51 & 0.9 & 0.62 & $\mathrm{~S}$ & $\mathrm{EW}$ & 1000 & $\mathrm{sig}$ \\
c5 & 22 & 1 & 304 & 35 & 0.96 & 0.39 & 0.0 & 0.39 & 0.9 & 0.55 & $\mathrm{~S}$ & $\mathrm{EF}$ & 1500 & $\mathrm{rbf}$ \\
c6 & 21 & 0 & 305 & 36 & 1.0 & 0.37 & 0.0 & 0.37 & 0.9 & 0.54 & - & $\mathrm{EF}$ & 500 & sig \\
c7 & 21 & 0 & 305 & 36 & 1.0 & 0.37 & 0.0 & 0.37 & 0.9 & 0.54 & - & $\mathrm{EF}$ & 500 & sig \\
c8 & 20 & 0 & 305 & 37 & 1.0 & 0.35 & 0.0 & 0.35 & 0.9 & 0.52 & $\mathrm{~S}$ & $\mathrm{EF}$ & 1500 & sig \\
c9 & 31 & 33 & 272 & 26 & 0.48 & 0.54 & 0.11 & 0.54 & 0.84 & 0.51 & $\mathrm{~S}$ & $\mathrm{EW}$ & 500 & sig \\
\hline
\end{tabular}


Os desempenhos dos melhores modelos de classificação (antes e após aplicação da técnica PU) também podem ser comparados por meio da análise do espaço ROC, conforme apresenta a figura 14. Nota-se que, após a estimação dos rótulos via PU, os pontos relativos aos classificadores avaliados moveram-se de áreas pouco favorecidas para áreas no espaço ROC associadas a melhores desempenhos.

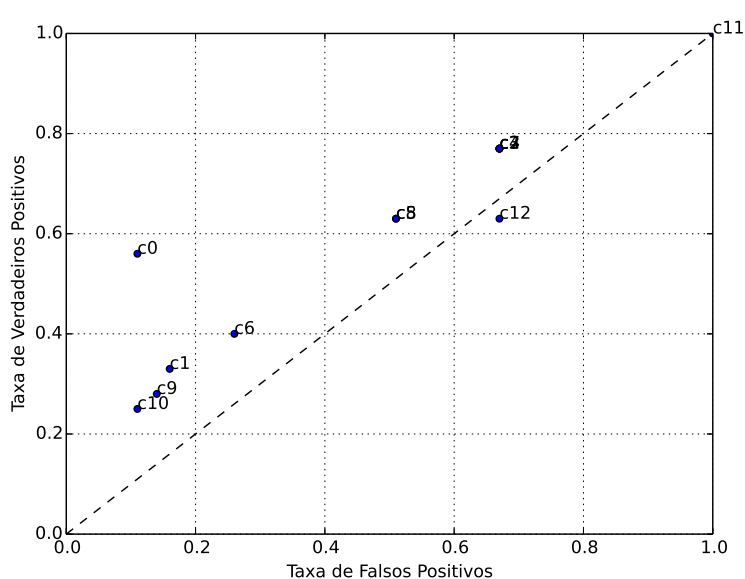

(a)

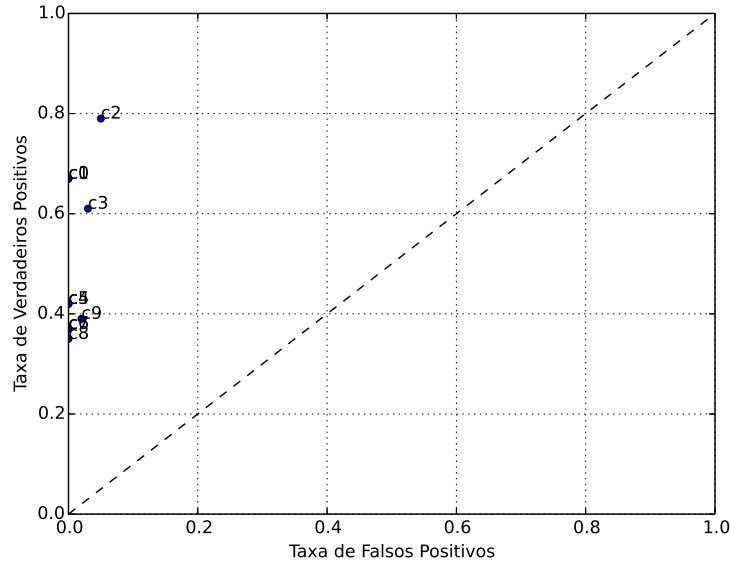

(b)

Figura 7 - Análise ROC para classificadores com fator de custo $C_{i j}=\frac{1}{\text { total }_{p}+\text { total }_{n}}$ para o conjunto de dados AID1284. (a) Para dados com rotulação original (b) Para dados com rotulação após PU.

Cenário 2: Análise de classificadores ELM com fator de custo $C_{i j}=\frac{0.5}{\text { total }_{p}+\text { total }_{n}}$

Aqui é apresentada uma análise comparativa entre os resultados obtidos pelo classificador ELM com fator de custo $C_{i j}=\frac{0.5}{\text { total }_{p}+\text { total }_{n}}$, considerando a rotulação original do conjunto AID1284 e a rotulação obtida após aplicação a técnica PU.

Os resultados alcançados pelo classificador construído para os dados com rotulação original são apresentados na tabela 12. O melhor resultado foi obtido para os dados padronizados e com discretização EF e para o modelo ELM com 500 neurônios e função de ativação sigmoidal. Nota-se que, com o fator de custo $C_{i j}=\frac{0.5}{\text { total }_{p}+\text { total }_{n}}$, obteve-se melhores resultados, em comparação aos alcançados com o custo $C_{i j}=\frac{1}{\text { total }_{p}+\text { total }_{n}}$. Além disso, o melhor desempenho foi observado para uma arquitetura de 500 neurônios, a menor considerada nos experimentos deste trabalho. 
Tabela 12 - Resultados obtidos pelo classificador ELM com fator de custo $C_{i j}=\frac{0.5}{\text { total }_{p}+\text { total }_{n}}$ para o conjunto de dados AID1284 com rotulação original.

\begin{tabular}{|c|c|c|c|c|c|c|c|c|c|c|c|c|c|c|}
\hline id & VP & FP & VN & FN & Pre. & Rec. & $t_{f p}$ & $t_{v p}$ & Acc & $f_{1}$ & Scale & Disc & Neu. & Fa. \\
\hline c0 & 63 & 18 & 592 & 51 & 0.78 & 0.55 & 0.03 & 0.55 & 0.9 & 0.65 & $\mathrm{~S}$ & EF & 500 & sig \\
c1 & 52 & 8 & 602 & 62 & 0.87 & 0.46 & 0.01 & 0.46 & 0.9 & 0.6 & $\mathrm{~S}$ & $\mathrm{EF}$ & 1000 & $\mathrm{sig}$ \\
$\mathrm{c} 2$ & 36 & 10 & 600 & 78 & 0.78 & 0.32 & 0.02 & 0.32 & 0.88 & 0.45 & $\mathrm{~S}$ & $\mathrm{EF}$ & 1000 & $\mathrm{rbf}$ \\
c3 & 35 & 8 & 602 & 79 & 0.81 & 0.31 & 0.01 & 0.31 & 0.88 & 0.45 & $\mathrm{~S}$ & $\mathrm{EF}$ & 1500 & $\mathrm{sig}$ \\
c4 & 58 & 135 & 475 & 56 & 0.3 & 0.51 & 0.22 & 0.51 & 0.74 & 0.38 & $\mathrm{~S}$ & $\mathrm{EF}$ & 1500 & $\mathrm{sig}$ \\
c5 & 54 & 132 & 478 & 60 & 0.29 & 0.47 & 0.22 & 0.47 & 0.73 & 0.36 & $\mathrm{~S}$ & $\mathrm{EW}$ & 1500 & $\mathrm{sig}$ \\
c6 & 51 & 169 & 441 & 63 & 0.23 & 0.45 & 0.28 & 0.45 & 0.68 & 0.31 & $\mathrm{~S}$ & $\mathrm{EF}$ & 1000 & $\mathrm{sig}$ \\
c7 & 22 & 7 & 603 & 92 & 0.76 & 0.19 & 0.01 & 0.19 & 0.86 & 0.31 & $\mathrm{~S}$ & $\mathrm{EF}$ & 1500 & $\mathrm{rbf}$ \\
c8 & 21 & 6 & 604 & 93 & 0.78 & 0.18 & 0.01 & 0.18 & 0.86 & 0.3 & - & $\mathrm{EW}$ & 1000 & $\mathrm{sig}$ \\
$\mathrm{c} 9$ & 30 & 73 & 537 & 84 & 0.29 & 0.26 & 0.12 & 0.26 & 0.78 & 0.28 & $\mathrm{~S}$ & - & 500 & $\mathrm{sig}$ \\
\hline
\end{tabular}

Após a aplicação da técnica PU, novos classificadores foram construídos e seus resultados (ver tabela 13) foram analisados. Observa-se aqui que o modelo ELM aumentou a sua acurácia de $90 \%$ para $95 \%$, entretanto, o melhor resultado requereu um número maior, de 1500 neurônios na camada escondida, em comparação aos experimentos com rotulação original. Apesar do melhor modelo ter utilizado dados com discretização do tipo EW e padronização, os demais modelos com desempenho similares contemplam o método de discretização EF. Contudo, todos estes apresentam unidades com função sigmoidal.

Outros destaques destes experimentos são a diminuição no número de falsos negativos, de 51 para 13 e a diminuição no número de falsos positivos, de 18 para 4 .

Tabela 13 - Resultados obtidos pelo classificador ELM com fator de custo $C_{i j}=\frac{0.5}{\text { total }_{p}+\text { total }_{n}}$ para o conjunto de dados AID1284 após a aplicação do rotulador PU.

\begin{tabular}{|c|c|c|c|c|c|c|c|c|c|c|c|c|c|c|}
\hline id & VP & FP & VN & FN & Pre. & Rec. & $t_{f p}$ & $t_{v p}$ & Acc & $f_{1}$ & Scale & Disc & Neu. & Fa. \\
\hline c0 & 44 & 4 & 301 & 13 & 0.92 & 0.77 & 0.01 & 0.77 & 0.95 & 0.84 & $\mathrm{~S}$ & $\mathrm{EW}$ & 1500 & sig \\
c1 & 38 & 3 & 302 & 19 & 0.93 & 0.67 & 0.01 & 0.67 & 0.94 & 0.78 & - & $\mathrm{EF}$ & 1500 & sig \\
c2 & 38 & 3 & 302 & 19 & 0.93 & 0.67 & 0.01 & 0.67 & 0.94 & 0.78 & - & $\mathrm{EF}$ & 1500 & sig \\
c3 & 23 & 4 & 301 & 34 & 0.85 & 0.4 & 0.01 & 0.4 & 0.9 & 0.55 & - & $\mathrm{EF}$ & 500 & sig \\
c4 & 23 & 4 & 301 & 34 & 0.85 & 0.4 & 0.01 & 0.4 & 0.9 & 0.55 & - & $\mathrm{EF}$ & 500 & sig \\
c5 & 52 & 89 & 216 & 5 & 0.37 & 0.91 & 0.29 & 0.91 & 0.74 & 0.53 & - & $\mathrm{EW}$ & 1500 & sig \\
c6 & 20 & 1 & 304 & 37 & 0.95 & 0.35 & 0.0 & 0.35 & 0.9 & 0.51 & - & $\mathrm{EF}$ & 1000 & sig \\
c7 & 20 & 1 & 304 & 37 & 0.95 & 0.35 & 0.0 & 0.35 & 0.9 & 0.51 & - & $\mathrm{EF}$ & 1000 & sig \\
c8 & 19 & 1 & 304 & 38 & 0.95 & 0.33 & 0.0 & 0.33 & 0.89 & 0.49 & - & $\mathrm{EW}$ & 1000 & sig \\
c9 & 19 & 1 & 304 & 38 & 0.95 & 0.33 & 0.0 & 0.33 & 0.89 & 0.49 & - & $\mathrm{EW}$ & 1500 & sig \\
\hline
\end{tabular}

Os desempenhos dos melhores modelos de classificação (antes e após aplicação da técnica PU) também podem ser comparados por meio da análise do espaço ROC, conforme apresenta a figura 8. Nota-se que, após a estimação dos rótulos via PU, os pontos 
relativos aos classificadores avaliados moveram-se de áreas pouco favorecidas para áreas no espaço ROC associadas a melhores desempenhos. É possível perceber,ainda, que os modelos selecionados antes da etapa de estimação dos rótulos PU acertaram menos de $60 \%$ dos elementos positivos e, em alguns casos, obtiveram uma taxa de falso positivos próxima a 30\%. Com base nas rotulações estimadas, os melhores modelos puderam acertar cerca de $80 \%$ dos elementos positivos existentes no conjunto.

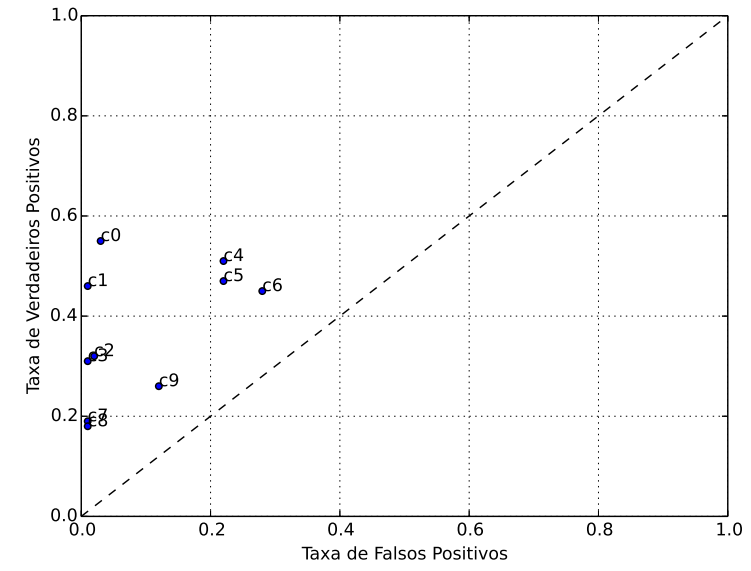

(a)

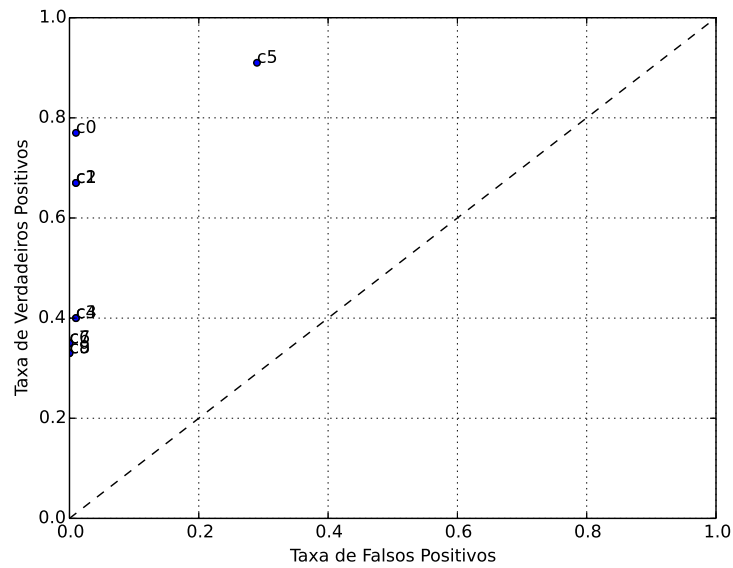

(b)

Figura 8 - Análise ROC para classificadores com fator de custo $C_{i j}=\frac{0.5}{\text { total }_{p}+\text { total }_{n}}$ para o conjunto de dados AID1284. (a) Para dados com rotulação original (b) Para dados com rotulação após PU.

Cenário 3: Análise de classificadores ELM com fator de custo 1.0

Aqui é apresentada uma análise comparativa entre os resultados obtidos pelo classificador ELM com fator de custo 1.0, considerando a rotulação original do conjunto AID1284 e a rotulação obtida após aplicação a técnica PU.

Os resultados alcançados pelo classificador construído para os dados com rotulação original são apresentados na tabela 14. O melhor resultado foi obtido para os dados padronizados, sem discretização e modelo ELM com 1500 neurônios e função de ativação sigmoidal. Nota-se, entretanto, que esse desempenho está abaixo do erro majoritário para o conjunto, que é igual a $84,25 \%$ e, portanto, considerado insatisfatório. 
Tabela 14 - Resultados obtidos pelo classificador ELM com fator de custo 1.0 para o conjunto de dados AID1284 antes da aplicação do rotulador PU.

\begin{tabular}{|c|c|c|c|c|c|c|c|c|c|c|c|c|c|c|}
\hline id & VP & FP & VN & FN & Pre. & Rec. & $t_{f p}$ & $t_{v p}$ & Acc & $f_{1}$ & Scale & Disc & Neu. & Fa. \\
\hline c0 & 20 & 26 & 279 & 37 & 0.43 & 0.35 & 0.09 & 0.35 & 0.83 & 0.39 & $\mathrm{~S}$ & - & 1500 & sig \\
c1 & 20 & 26 & 279 & 37 & 0.43 & 0.35 & 0.09 & 0.35 & 0.83 & 0.39 & $\mathrm{~S}$ & - & 1500 & sig \\
c2 & 44 & 207 & 98 & 13 & 0.18 & 0.77 & 0.68 & 0.77 & 0.39 & 0.29 & $\mathrm{~S}$ & $\mathrm{EF}$ & 1000 & lin \\
c3 & 42 & 200 & 105 & 15 & 0.17 & 0.74 & 0.66 & 0.74 & 0.41 & 0.28 & $\mathrm{~S}$ & - & 500 & lin \\
c4 & 44 & 210 & 95 & 13 & 0.17 & 0.77 & 0.69 & 0.77 & 0.38 & 0.28 & - & - & 1000 & rbf \\
c5 & 52 & 261 & 44 & 5 & 0.17 & 0.91 & 0.86 & 0.91 & 0.27 & 0.28 & - & - & 1500 & rbf \\
c6 & 42 & 200 & 105 & 15 & 0.17 & 0.74 & 0.66 & 0.74 & 0.41 & 0.28 & $\mathrm{~S}$ & - & 500 & lin \\
c7 & 56 & 305 & 0 & 1 & 0.16 & 0.98 & 1.0 & 0.98 & 0.15 & 0.27 & - & - & 1000 & rbf \\
c8 & 57 & 305 & 0 & 0 & 0.16 & 1.0 & 1.0 & 1.0 & 0.16 & 0.27 & - & - & 1500 & rbf \\
c9 & 57 & 305 & 0 & 0 & 0.16 & 1.0 & 1.0 & 1.0 & 0.16 & 0.27 & - & - & 500 & rbf \\
\hline
\end{tabular}

Após a aplicação da técnica PU, novos classificadores foram construídos e seus resultados (ver tabela 15) foram analisados. Nesses experimentos, o modelo atingiu uma acurácia de 95\%, para dados sem padronização, discretização EF e modelo com 1500 neurônios e função de ativação sigmoidal na camada escondida. Tal resultado representa uma melhora significativa de desempenho em comparação àqueles obtidos com rotulação original. Outros destaques destes experimentos são a inexistência de falsos positivos e a diminuição no número de falsos negativos, de 37 para 19.

Tabela 15 - Resultados obtidos pelo classificador ELM com fator de custo 1.0 para o conjunto de dados AID1284 após a aplicação do rotulador PU.

\begin{tabular}{|c|c|c|c|c|c|c|c|c|c|c|c|c|c|c|}
\hline id & VP & FP & VN & FN & Pre. & Rec. & $t_{f p}$ & $t_{v p}$ & Acc & $f_{1}$ & Scale & Disc & Neu. & Fa. \\
\hline c0 & 38 & 0 & 305 & 19 & 1.0 & 0.67 & 0.0 & 0.67 & 0.95 & 0.8 & - & EF & 1500 & sig \\
c1 & 38 & 0 & 305 & 19 & 1.0 & 0.67 & 0.0 & 0.67 & 0.95 & 0.8 & - & EF & 1500 & sig \\
c2 & 45 & 14 & 291 & 12 & 0.76 & 0.79 & 0.05 & 0.79 & 0.93 & 0.78 & $\mathrm{~S}$ & EW & 1500 & rbf \\
c3 & 35 & 9 & 296 & 22 & 0.8 & 0.61 & 0.03 & 0.61 & 0.91 & 0.69 & $\mathrm{~S}$ & $\mathrm{EF}$ & 1500 & sig \\
c4 & 24 & 0 & 305 & 33 & 1.0 & 0.42 & 0.0 & 0.42 & 0.91 & 0.59 & - & $\mathrm{EF}$ & 1000 & sig \\
c5 & 24 & 0 & 305 & 33 & 1.0 & 0.42 & 0.0 & 0.42 & 0.91 & 0.59 & - & $\mathrm{EF}$ & 1000 & sig \\
c6 & 21 & 0 & 305 & 36 & 1.0 & 0.37 & 0.0 & 0.37 & 0.9 & 0.54 & - & $\mathrm{EF}$ & 500 & sig \\
c7 & 21 & 0 & 305 & 36 & 1.0 & 0.37 & 0.0 & 0.37 & 0.9 & 0.54 & - & $\mathrm{EF}$ & 500 & sig \\
c8 & 20 & 1 & 304 & 37 & 0.95 & 0.35 & 0.0 & 0.35 & 0.9 & 0.51 & - & $\mathrm{EW}$ & 1500 & sig \\
c9 & 22 & 7 & 298 & 35 & 0.76 & 0.39 & 0.02 & 0.39 & 0.88 & 0.51 & $\mathrm{~S}$ & $\mathrm{EF}$ & 1000 & sig \\
\hline
\end{tabular}

Os desempenhos dos melhores modelos de classificação (antes e após aplicação da técnica PU) também podem ser comparados por meio da análise do espaço ROC, conforme apresenta a figura 9. Nota-se que, após a estimação dos rótulos via PU, os pontos relativos aos classificadores avaliados moveram-se de áreas pouco favorecidas para áreas no espaço ROC associadas a melhores desempenhos. Percebe-se, ainda, que os dados em 
sua rotulação original produziram resultados próximos à abordagem dita aleatória, ou seja, estão próximos à linha tracejada do espaço ROC. Em relação à rotulação estimada, observa-se uma redução na taxa dos falsos positivos e uma mudança no comportamento dos modelos selecionados, os quais se mostraram mais conservadores em relação à rotulação estimada pelo processo PU.

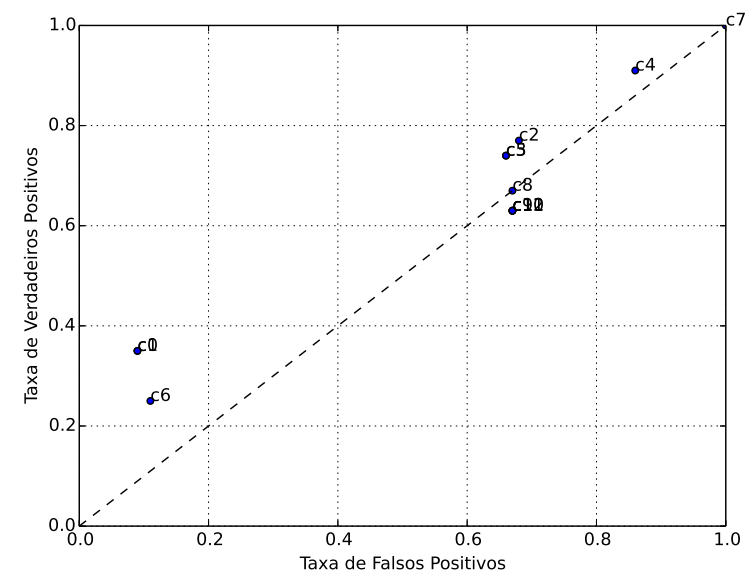

(a)

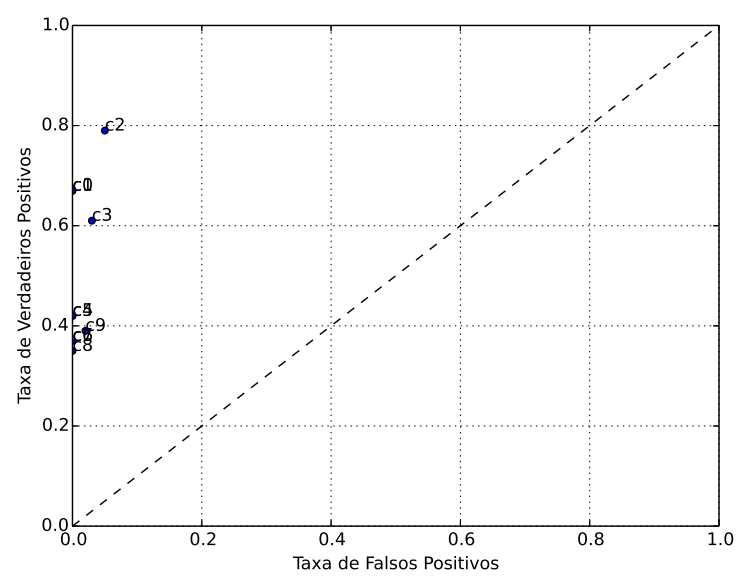

(b)

Figura 9 - Análise ROC para classificadores com fator de custo $C_{i j}=1.0$ para o conjunto de dados AID1284. (a) Para dados com rotulação original (b) Para dados com rotulação após PU.

Cenário 4: Análise de classificadores ELM com fator de custo $C_{i j}=1.0+\frac{1}{\text { total }_{p}+\text { total }_{n}}$

Aqui é apresentada uma análise comparativa entre os resultados obtidos pelo classificador ELM com fator de custo $C_{i j}=1.0+\frac{1}{\text { total }_{p}+\text { total }_{n}}$, considerando a rotulação original do conjunto AID1284 e a rotulação obtida após aplicação a técnica PU.

Os resultados alcançados pelo classificador construído para os dados com rotulação original são apresentados na tabela 16. O melhor resultado (acurácia igual a 88\%) foi obtido para os dados sem padronização, sem discretização e modelo ELM com 1000 neurônios e função de ativação sigmoidal. 
Tabela 16 - Resultados obtidos pelo classificador ELM com fator de custo $C_{i j}=1.0+$ $\frac{1}{\text { total }_{p}+\text { total }_{n}}$ para o conjunto de dados AID1284 antes da aplicação do rotulador PU.

\begin{tabular}{|c|c|c|c|c|c|c|c|c|c|c|c|c|c|c|}
\hline id & VP & FP & VN & FN & Pre. & Rec. & $t_{f p}$ & $t_{v p}$ & Acc & $f_{1}$ & Scale & Disc & Neu. & Fa. \\
\hline c0 & 12 & 0 & 305 & 45 & 1.0 & 0.21 & 0.0 & 0.21 & 0.88 & 0.35 & - & - & 1000 & sig \\
c1 & 11 & 0 & 305 & 46 & 1.0 & 0.19 & 0.0 & 0.19 & 0.87 & 0.32 & - & - & 1500 & sig \\
c2 & 10 & 2 & 303 & 47 & 0.83 & 0.18 & 0.01 & 0.18 & 0.86 & 0.29 & - & - & 500 & sig \\
c3 & 47 & 227 & 78 & 10 & 0.17 & 0.82 & 0.74 & 0.82 & 0.35 & 0.28 & $\mathrm{~S}$ & - & 1500 & lin \\
c4 & 52 & 265 & 40 & 5 & 0.16 & 0.91 & 0.87 & 0.91 & 0.25 & 0.28 & $\mathrm{~S}$ & - & 1500 & lin \\
c5 & 52 & 265 & 40 & 5 & 0.16 & 0.91 & 0.87 & 0.91 & 0.25 & 0.28 & $\mathrm{~S}$ & - & 1500 & lin \\
c6 & 47 & 227 & 78 & 10 & 0.17 & 0.82 & 0.74 & 0.82 & 0.35 & 0.28 & $\mathrm{~S}$ & - & 1500 & lin \\
c7 & 29 & 122 & 183 & 28 & 0.19 & 0.51 & 0.4 & 0.51 & 0.59 & 0.28 & $\mathrm{~S}$ & EW & 1500 & sig \\
c8 & 56 & 302 & 3 & 1 & 0.16 & 0.98 & 0.99 & 0.98 & 0.16 & 0.27 & - & - & 1000 & rbf \\
c9 & 57 & 305 & 0 & 0 & 0.16 & 1.0 & 1.0 & 1.0 & 0.16 & 0.27 & - & - & 1500 & rbf \\
\hline
\end{tabular}

Após a aplicação da técnica PU, novos classificadores foram construídos e seus resultados (ver tabela 17) foram analisados. Nesses experimentos, o melhor modelo utiliza discretização EF, padronização e função sigmoidal com 1000 neurônios na camada escondida. O modelo atingiu acurácia de 95\% sem apresentar nenhum elemento positivo classificado incorretamente. Além disso, o número de falsos negativos caiu de 45 para 18.

Tabela 17 - Resultados obtidos pelo classificador ELM com fator de custo $C_{i j}=1.0+$ $\frac{1}{\text { total }_{p}+\text { total }_{n}}$ para o conjunto de dados AID1284 após a aplicação do rotulador PU.

\begin{tabular}{|c|c|c|c|c|c|c|c|c|c|c|c|c|c|c|}
\hline id & VP & FP & VN & FN & Pre. & Rec. & $t_{f p}$ & $t_{v p}$ & Acc & $f_{1}$ & Scale & Disc & Neu. & Fa. \\
\hline c0 & 39 & 0 & 305 & 18 & 1.0 & 0.68 & 0.0 & 0.68 & 0.95 & 0.81 & $\mathrm{~S}$ & $\mathrm{EF}$ & 1000 & $\mathrm{sig}$ \\
c1 & 39 & 12 & 293 & 18 & 0.76 & 0.68 & 0.04 & 0.68 & 0.92 & 0.72 & $\mathrm{~S}$ & $\mathrm{EW}$ & 1500 & $\mathrm{sig}$ \\
c2 & 33 & 8 & 297 & 24 & 0.8 & 0.58 & 0.03 & 0.58 & 0.91 & 0.67 & $\mathrm{~S}$ & $\mathrm{EF}$ & 1500 & $\mathrm{sig}$ \\
c3 & 36 & 14 & 291 & 21 & 0.72 & 0.63 & 0.05 & 0.63 & 0.9 & 0.67 & $\mathrm{~S}$ & $\mathrm{EW}$ & 1000 & $\mathrm{sig}$ \\
c4 & 20 & 0 & 305 & 37 & 1.0 & 0.35 & 0.0 & 0.35 & 0.9 & 0.52 & $\mathrm{~S}$ & $\mathrm{EF}$ & 1500 & $\mathrm{sig}$ \\
c5 & 20 & 0 & 305 & 37 & 1.0 & 0.35 & 0.0 & 0.35 & 0.9 & 0.52 & - & $\mathrm{EF}$ & 1500 & $\mathrm{rbf}$ \\
c6 & 20 & 0 & 305 & 37 & 1.0 & 0.35 & 0.0 & 0.35 & 0.9 & 0.52 & - & $\mathrm{EF}$ & 500 & $\mathrm{rbf}$ \\
c7 & 20 & 0 & 305 & 37 & 1.0 & 0.35 & 0.0 & 0.35 & 0.9 & 0.52 & - & $\mathrm{EF}$ & 1500 & $\mathrm{rbf}$ \\
c8 & 20 & 0 & 305 & 37 & 1.0 & 0.35 & 0.0 & 0.35 & 0.9 & 0.52 & - & $\mathrm{EF}$ & 500 & $\mathrm{rbf}$ \\
c9 & 19 & 0 & 305 & 38 & 1.0 & 0.33 & 0.0 & 0.33 & 0.9 & 0.5 & - & $\mathrm{EF}$ & 1000 & sig \\
\hline
\end{tabular}

Os desempenhos dos melhores modelos de classificação (antes e após aplicação da técnica PU) também podem ser comparados por meio da análise do espaço ROC, conforme apresenta a figura 10. Nota-se que, após a estimação dos rótulos via PU, os pontos relativos aos classificadores avaliados moveram-se de áreas pouco favorecidas para áreas no espaço ROC associadas a melhores desempenhos. É possível observar que o melhor classificador, aqui identificado com c0, é posicionado em uma área do gráfico dita conservadora. Mais 
especificamente, o melhor classificador necessita de mais evidências para classificar uma instância como positiva, justificando assim a baixa taxa de falsos positivos.

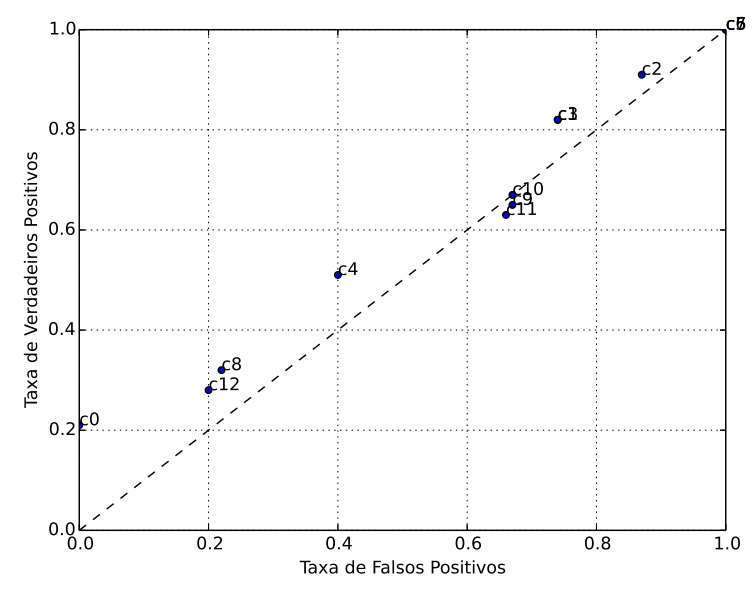

(a)

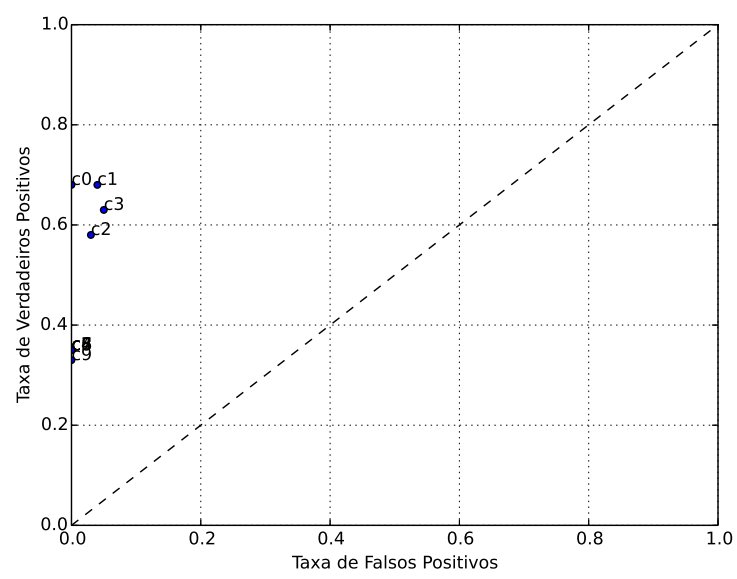

(b)

Figura 10 - Análise ROC para classificadores com fator de custo $C_{i j}=1.0+\frac{\text { total }_{p}}{\text { total }_{n}}$ para conjunto de dados AID1284. (a) Para dados com rotulação original (b) Para dados com rotulação após PU.

Em uma análise geral para os experimentos com o conjunto de dados AID1284, observa-se que a discretização EW produziu bons resultados quando utilizada com padronização. Por outro lado, a técnica EF aparece junto a alguns modelos com bom desempenho, mesmo sem a etapa de padronização no pré-processamento. Para esse conjunto de dados, os melhores resultados foram encontrados para custo $C_{i j}=0.5+\frac{\text { total }_{p}}{\text { total }_{n}}$, dados padronizados e discretização EW, atingindo 95\% de acerto com 1500 neurônios e função sigmoidal na configuração do classificador, resultado este acima de seu erro majoritário de 84,25\%.

\subsubsection{Experimentos de rotulação via PU - Conjunto de dados AIDAID721}

De forma análoga aos experimentos anteriores, o conjunto de dados AID1721 foi submetido à etapa de rotulação PU. A tabela 18 apresenta as alterações ocorridas na distribuição dos dados após o processo PU, considerando a rotulação original de 21 elementos positivos e 73 elementos negativos. 
Tabela 18 - Distribuições de classes obtidas após a execução do processo PU contemplando a variação dos custos para o conjunto de dados AID721.

\begin{tabular}{|c|c|c|c|c|}
\hline $\begin{array}{ll}\text { Conjunto de dados } & \text { Custo } \\
\end{array}$ & $\frac{1}{\text { total }_{p}+\text { total }_{n}}$ & $0.5+\frac{\text { total }_{p}}{\text { total }_{n}}$ & 1.0 & $1.0+\frac{\text { total }_{p}}{\text { total }_{n}}$ \\
\hline Bin. e Cont. + EW & $86 / 8$ & $73 / 21$ & $35 / 59$ & $21 / 73$ \\
\hline Bin. & $75 / 19$ & $35 / 59$ & $29 / 65$ & $27 / 67$ \\
\hline Bin. e Cont. & $21 / 73$ & $33 / 61$ & $31 / 63$ & $29 / 65$ \\
\hline Cont. & $21 / 73$ & $40 / 54$ & $46 / 48$ & $41 / 53$ \\
\hline Bin. e Cont. + Scale & $57 / 305$ & $60 / 302$ & $60 / 302$ & $62 / 300$ \\
\hline Cont. + Scale & $57 / 305$ & $102 / 260$ & $58 / 304$ & $58 / 304$ \\
\hline Bin. e Cont. + EF & $43 / 51$ & $29 / 65$ & $26 / 68$ & $28 / 66$ \\
\hline Bin. e Cont. + scale + EF & $60 / 34$ & $37 / 57$ & $26 / 68$ & $26 / 68$ \\
\hline Cont. + EF & $21 / 73$ & $27 / 67$ & $37 / 57$ & $26 / 68$ \\
\hline Cont. + scale + EW & $60 / 34$ & $25 / 69$ & $22 / 72$ & $40 / 54$ \\
\hline Cont. + scale + EF & $45 / 49$ & $24 / 70$ & $28 / 66$ & $36 / 58$ \\
\hline Bin. e Cont + scale + EW & $46 / 48$ & $21 / 73$ & $21 / 73$ & $21 / 73$ \\
\hline Cont. + EW & $67 / 27$ & $24 / 70$ & $24 / 70$ & $25 / 69$ \\
\hline
\end{tabular}

Em relação aos resultados apresentados percebe-se que, no que diz respeito aos fatores de custo, nos casos nos quais $C_{i j}=1.0+\frac{t_{\text {otal }}}{\text { total }_{n}}$, a rotulação sofre poucas alterações. Em outros casos em que a rotulação não se altera, é possível perceber também o uso do método de discretização EW.

\subsubsection{Experimentos de classificação via ELM - Conjunto de dados AID721}

Nos próximos experimentos, classificadores ELM serão construídos para o conjunto de dados AID721, levando em conta sua rotulação original e as rotulações estimadas pelo método PU. Objetiva-se, com essa análise, avaliar o quanto esses diferentes cenários afetam o desempenho final do modelo de classificação.

Cenário 1: Análise de classificadores ELM com fator de custo $C_{i j}=\frac{1}{\text { total }_{p}+\text { total }_{n}}$

Os resultados alcançados pelo classificador construído para os dados com rotulação original são apresentados na tabela 16. O melhor resultado (acurácia igual a 89\%) foi obtido para os dados com padronização, sem discretização e modelo ELM com 1500 neurônios e função de ativação RBF. 
Tabela 19 - Resultados obtidos pelo classificador ELM com fator de custo $C_{i j}=\frac{1}{\text { total }_{p}+\text { total }_{n}}$ para o conjunto de dados AID721 antes da aplicação do rotulador PU.

\begin{tabular}{|c|c|c|c|c|c|c|c|c|c|c|c|c|c|c|}
\hline id & VP & FP & VN & FN & Pre. & Rec. & $t_{f p}$ & $t_{v p}$ & Acc & $f_{1}$ & Scale & Disc & Neu. & Fa. \\
\hline c0 & 14 & 3 & 70 & 7 & 0.82 & 0.67 & 0.04 & 0.67 & 0.89 & 0.74 & $\mathrm{~S}$ & - & 1500 & rbf \\
c1 & 14 & 3 & 70 & 7 & 0.82 & 0.67 & 0.04 & 0.67 & 0.89 & 0.74 & $\mathrm{~S}$ & - & 1500 & rbf \\
c2 & 13 & 2 & 71 & 8 & 0.87 & 0.62 & 0.03 & 0.62 & 0.89 & 0.72 & $\mathrm{~S}$ & - & 1000 & sig \\
c3 & 13 & 2 & 71 & 8 & 0.87 & 0.62 & 0.03 & 0.62 & 0.89 & 0.72 & $\mathrm{~S}$ & - & 1000 & sig \\
c4 & 18 & 26 & 47 & 3 & 0.41 & 0.86 & 0.36 & 0.86 & 0.69 & 0.55 & $\mathrm{~S}$ & $\mathrm{EW}$ & 500 & lin \\
c5 & 7 & 0 & 73 & 14 & 1.0 & 0.33 & 0.0 & 0.33 & 0.85 & 0.5 & $\mathrm{~S}$ & $\mathrm{EW}$ & 500 & $\mathrm{rbf}$ \\
c6 & 8 & 5 & 68 & 13 & 0.62 & 0.38 & 0.07 & 0.38 & 0.81 & 0.47 & $\mathrm{~S}$ & $\mathrm{EF}$ & 1000 & sig \\
c7 & 7 & 4 & 69 & 14 & 0.64 & 0.33 & 0.05 & 0.33 & 0.81 & 0.44 & $\mathrm{~S}$ & $\mathrm{EW}$ & 1500 & sig \\
c8 & 6 & 1 & 72 & 15 & 0.86 & 0.29 & 0.01 & 0.29 & 0.83 & 0.43 & $\mathrm{~S}$ & - & 1000 & rbf \\
c9 & 10 & 15 & 58 & 11 & 0.4 & 0.48 & 0.21 & 0.48 & 0.72 & 0.43 & $\mathrm{~S}$ & - & 500 & sig \\
\hline
\end{tabular}

Após a aplicação da técnica PU, novos classificadores foram construídos e seus resultados (ver tabela 17) foram analisados. Para esses experimentos de classificação utilizando ELM, nenhum dos modelos selecionados aplicou padronização em suas etapas de pré-processamento. Mesmo assim, o melhor desempenho atingiu 94\% de acurácia. Observase também a presença de modelos com funções sigmoidais e técnicas de discretização EW em sua maioria.

Tabela 20 - Resultados obtidos pelo classificador ELM com fator de custo $C_{i j}=\frac{1}{\text { total }_{p}+\text { total }_{n}}$ para o conjunto de dados AID721 após a aplicação do rotulador PU.

\begin{tabular}{|c|c|c|c|c|c|c|c|c|c|c|c|c|c|c|}
\hline id & VP & FP & VN & FN & Pre. & Rec. & $t_{f p}$ & $t_{v p}$ & Acc & $f_{1}$ & Scale & Disc & Neu. & Fa. \\
\hline c0 & 15 & 0 & 73 & 6 & 1.0 & 0.71 & 0.0 & 0.71 & 0.94 & 0.83 & - & EW & 1500 & sig \\
c1 & 15 & 0 & 73 & 6 & 1.0 & 0.71 & 0.0 & 0.71 & 0.94 & 0.83 & - & EW & 1500 & sig \\
c2 & 14 & 0 & 73 & 7 & 1.0 & 0.67 & 0.0 & 0.67 & 0.93 & 0.8 & - & EW & 1000 & sig \\
c3 & 14 & 0 & 73 & 7 & 1.0 & 0.67 & 0.0 & 0.67 & 0.93 & 0.8 & - & EW & 500 & sig \\
c4 & 14 & 0 & 73 & 7 & 1.0 & 0.67 & 0.0 & 0.67 & 0.93 & 0.8 & - & EW & 1000 & sig \\
c5 & 14 & 0 & 73 & 7 & 1.0 & 0.67 & 0.0 & 0.67 & 0.93 & 0.8 & - & EW & 500 & sig \\
c6 & 13 & 0 & 73 & 8 & 1.0 & 0.62 & 0.0 & 0.62 & 0.91 & 0.76 & - & EF & 500 & lin \\
c7 & 17 & 7 & 66 & 4 & 0.71 & 0.81 & 0.1 & 0.81 & 0.88 & 0.76 & - & EW & 1000 & sig \\
c8 & 13 & 0 & 73 & 8 & 1.0 & 0.62 & 0.0 & 0.62 & 0.91 & 0.76 & - & EF & 500 & lin \\
c9 & 12 & 1 & 72 & 9 & 0.92 & 0.57 & 0.01 & 0.57 & 0.89 & 0.71 & - & EW & 1500 & sig \\
\hline
\end{tabular}

Os desempenhos dos melhores modelos de classificação (antes e após aplicação da técnica PU) também podem ser comparados por meio da análise do espaço ROC, conforme apresenta a figura 11. Nota-se que, após a estimação dos rótulos via PU, os pontos relativos aos classificadores avaliados moveram-se de áreas pouco favorecidas para áreas no espaço ROC associadas a melhores desempenhos. A análise dos classificadores indica, ainda, que para os dados os quais não foi empregado o processo de estimação de rótulos PU, os 
modelos selecionados não atingiram marcas expressivas, além das baixas taxas de falsos positivos. Mesmo após a aplicação do processo PU, observa-se que os modelos ainda se conservaram, em sua maioria, abaixo dos $80 \%$ em relação a taxa de verdadeiros positivos.

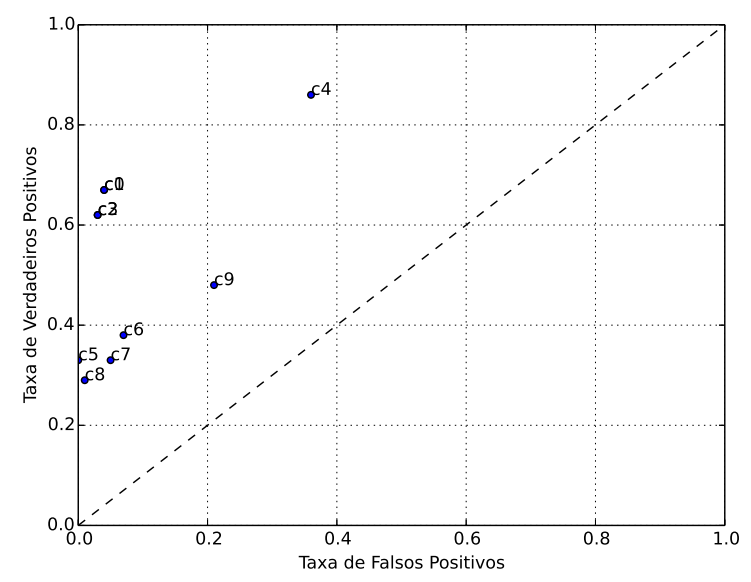

(a)

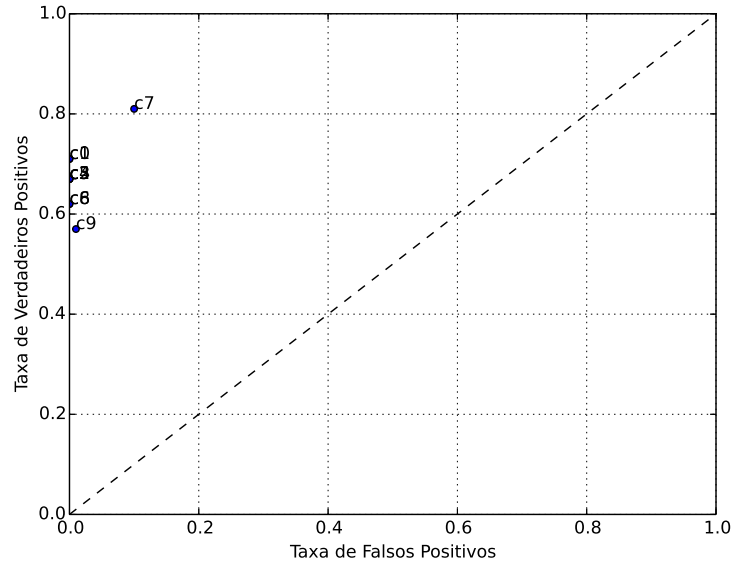

(b)

Figura 11 - Análise ROC para classificadores com fator de custo $C_{i j}=\frac{1}{\text { total }_{p}+\text { total }_{n}}$ para conjunto de dados AID721. (a) Para dados com rotulação original (b) Para dados com rotulação após PU.

Cenário 2: Análise de classificadores ELM com fator de custo $C_{i j}=0.5+\frac{1}{\text { total }_{p}+\text { total }_{n}}$

Os resultados alcançados pelo classificador construído para os dados com rotulação original são apresentados na tabela 21. O melhor resultado (acurácia igual a 91\%) foi obtido para os dados sem padronização, com discretização EW e modelo ELM com 1500 neurônios e função de ativação RBF.

Tabela 21 - Resultados obtidos pelo classificador ELM comfator de custo $C_{i j}=0.5+$ $\frac{1}{\text { total }_{p}+\text { total }_{n}}$ para o conjunto de dados AID721 antes da aplicação do rotulador PU.

\begin{tabular}{|c|c|c|c|c|c|c|c|c|c|c|c|c|c|c|}
\hline id & VP & FP & VN & FN & Pre. & Rec. & $t_{f p}$ & $t_{v p}$ & Acc & $f_{1}$ & Scale & Disc & Neu. & Fa. \\
\hline c0 & 13 & 0 & 73 & 8 & 1.0 & 0.62 & 0.0 & 0.62 & 0.91 & 0.76 & - & EW & 1500 & rbf \\
c1 & 12 & 0 & 73 & 9 & 1.0 & 0.57 & 0.0 & 0.57 & 0.9 & 0.73 & - & EW & 1000 & rbf \\
c2 & 10 & 0 & 73 & 11 & 1.0 & 0.48 & 0.0 & 0.48 & 0.88 & 0.65 & - & EW & 500 & rbf \\
c3 & 14 & 23 & 50 & 7 & 0.38 & 0.67 & 0.32 & 0.67 & 0.68 & 0.48 & $\mathrm{~S}$ & EF & 500 & sig \\
c4 & 11 & 21 & 52 & 10 & 0.34 & 0.52 & 0.29 & 0.52 & 0.67 & 0.42 & $\mathrm{~S}$ & $\mathrm{EF}$ & 1500 & rbf \\
c5 & 17 & 45 & 28 & 4 & 0.27 & 0.81 & 0.62 & 0.81 & 0.48 & 0.41 & - & - & 1500 & lin \\
c6 & 12 & 26 & 47 & 9 & 0.32 & 0.57 & 0.36 & 0.57 & 0.63 & 0.41 & $\mathrm{~S}$ & $\mathrm{EF}$ & 1000 & lin \\
c7 & 20 & 58 & 15 & 1 & 0.26 & 0.95 & 0.79 & 0.95 & 0.37 & 0.4 & $\mathrm{~S}$ & - & 1500 & lin \\
c8 & 20 & 58 & 15 & 1 & 0.26 & 0.95 & 0.79 & 0.95 & 0.37 & 0.4 & $\mathrm{~S}$ & - & 1500 & lin \\
c9 & 17 & 48 & 25 & 4 & 0.26 & 0.81 & 0.66 & 0.81 & 0.45 & 0.4 & - & - & 1000 & lin \\
\hline
\end{tabular}


Após a aplicação da técnica PU, novos classificadores foram construídos e seus resultados (ver tabela 22) foram analisados. Para esses experimentos, a técnica de discretização EF e funções de ativação lineares foram predominantes dentre os modelos selecionados. Contudo, o melhor modelo atingiu somente $89 \%$ de acurácia mantendo o número de falsos positivos em zero. Nesse caso, nota-se que a aplicação da técnica PU levou a uma piora, em relação aos dois melhores resultados obtidos. Entretando para os outros modelos, a melhora decorrente da nova rotulação pode ser constatada.

Tabela 22 - Resultados obtidos pelo classificador ELM com fator de custo $C_{i j}=0.5+$ $\frac{1}{\text { total }_{p}+\text { total }_{n}}$ para o conjunto de dados AID721 após a aplicação do rotulador PU.

\begin{tabular}{|c|c|c|c|c|c|c|c|c|c|c|c|c|c|c|}
\hline id & VP & FP & VN & FN & Pre. & Rec. & $t_{f p}$ & $t_{v p}$ & Acc & $f_{1}$ & Scale & Disc & Neu. & Fa. \\
\hline c0 & 11 & 0 & 73 & 10 & 1.0 & 0.52 & 0.0 & 0.52 & 0.89 & 0.69 & - & EF & 1000 & lin \\
c1 & 11 & 0 & 73 & 10 & 1.0 & 0.52 & 0.0 & 0.52 & 0.89 & 0.69 & - & EF & 1000 & lin \\
c2 & 10 & 0 & 73 & 11 & 1.0 & 0.48 & 0.0 & 0.48 & 0.88 & 0.65 & - & EF & 500 & lin \\
c3 & 10 & 0 & 73 & 11 & 1.0 & 0.48 & 0.0 & 0.48 & 0.88 & 0.65 & - & EF & 500 & lin \\
c4 & 21 & 25 & 48 & 0 & 0.46 & 1.0 & 0.34 & 1.0 & 0.73 & 0.63 & $\mathrm{~S}$ & EF & 1500 & lin \\
c5 & 20 & 25 & 48 & 1 & 0.44 & 0.95 & 0.34 & 0.95 & 0.72 & 0.61 & $\mathrm{~S}$ & $\mathrm{EF}$ & 1000 & lin \\
c6 & 20 & 25 & 48 & 1 & 0.44 & 0.95 & 0.34 & 0.95 & 0.72 & 0.61 & $\mathrm{~S}$ & $\mathrm{EF}$ & 500 & lin \\
c7 & 11 & 4 & 69 & 10 & 0.73 & 0.52 & 0.05 & 0.52 & 0.85 & 0.61 & - & $\mathrm{EF}$ & 1500 & lin \\
c8 & 11 & 4 & 69 & 10 & 0.73 & 0.52 & 0.05 & 0.52 & 0.85 & 0.61 & - & $\mathrm{EF}$ & 1500 & lin \\
c9 & 10 & 2 & 71 & 11 & 0.83 & 0.48 & 0.03 & 0.48 & 0.86 & 0.61 & $\mathrm{~S}$ & $\mathrm{EW}$ & 500 & lin \\
\hline
\end{tabular}

Os desempenhos dos melhores modelos de classificação (antes e após aplicação da técnica PU) também podem ser comparados por meio da análise do espaço ROC, conforme apresenta a figura 12. Nota-se que, após a estimação dos rótulos via PU, os pontos relativos à maoiria dos classificadores avaliados moveram-se de áreas pouco favorecidas para áreas no espaço ROC associadas a melhores desempenhos, apesar da queda de desempenho para os dois melhores modelos selecionados. 


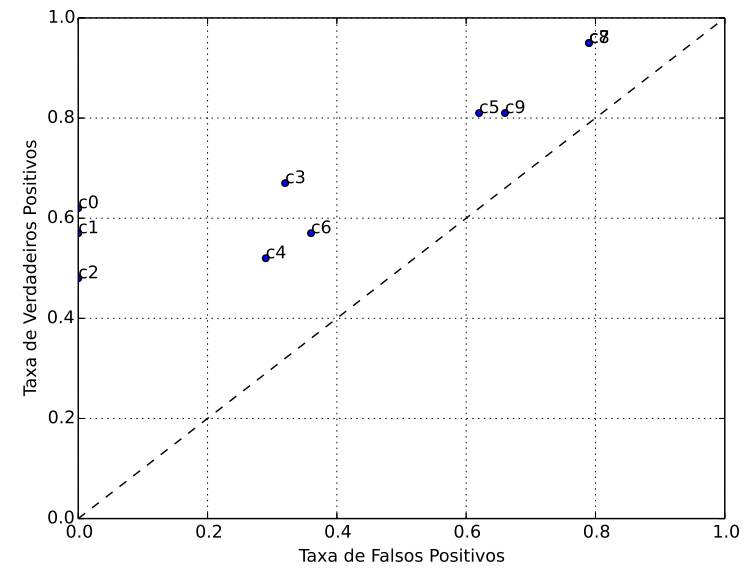

(a)

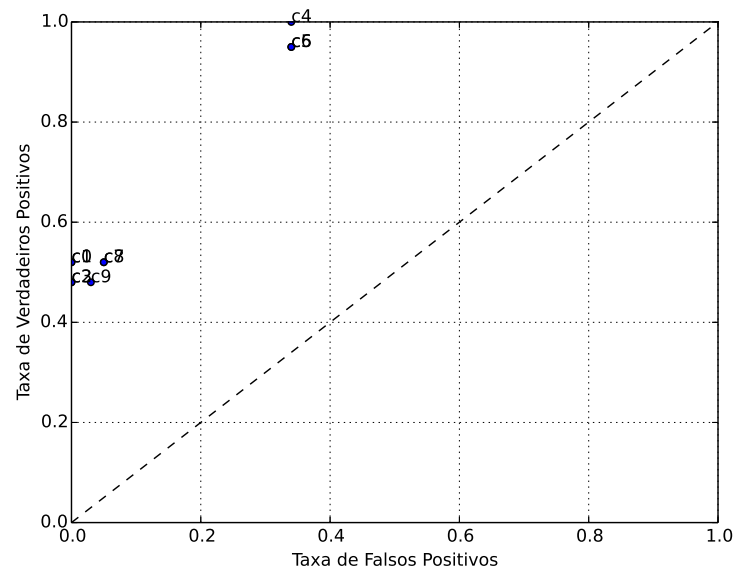

(b)

Figura 12 - Análise ROC para classificadores com fator de custo $C_{i j}=0.5+\frac{1}{\text { total }_{p}+\text { total }_{n}}$ para o conjunto de dados AID721. (a) Para dados com rotulação original (b) Para dados com rotulação após PU.

Cenário 3: Análise de classificadores ELM com fator de custo $C_{i j}=1.0$

Os resultados alcançados pelo classificador construído para os dados com rotulação original são apresentados na tabela 23. O melhor resultado (acurácia igual a 86\%) foi obtido para os dados sem padronização, com discretização EW e modelo ELM com 1000 neurônios e função de ativação RBF.

Tabela 23 - Resultados obtidos pelo classificador ELM com fator de custo 1.0 para o conjunto de dados AID721 antes da aplicação do rotulador PU.

\begin{tabular}{|c|c|c|c|c|c|c|c|c|c|c|c|c|c|c|}
\hline id & VP & FP & VN & FN & Pre. & Rec. & $t_{f p}$ & $t_{v p}$ & Acc & $f_{1}$ & Scale & Disc & Neu. & Fa. \\
\hline c0 & 14 & 6 & 67 & 7 & 0.7 & 0.67 & 0.08 & 0.67 & 0.86 & 0.68 & - & EW & 1000 & rbf \\
c1 & 14 & 6 & 67 & 7 & 0.7 & 0.67 & 0.08 & 0.67 & 0.86 & 0.68 & - & EW & 1500 & rbf \\
c2 & 14 & 6 & 67 & 7 & 0.7 & 0.67 & 0.08 & 0.67 & 0.86 & 0.68 & - & EW & 500 & rbf \\
c3 & 9 & 6 & 67 & 12 & 0.6 & 0.43 & 0.08 & 0.43 & 0.81 & 0.5 & $\mathrm{~S}$ & - & 1000 & sig \\
c4 & 9 & 6 & 67 & 12 & 0.6 & 0.43 & 0.08 & 0.43 & 0.81 & 0.5 & $\mathrm{~S}$ & - & 1000 & sig \\
c5 & 13 & 26 & 47 & 8 & 0.33 & 0.62 & 0.36 & 0.62 & 0.64 & 0.43 & - & EW & 1000 & lin \\
c6 & 10 & 18 & 55 & 11 & 0.36 & 0.48 & 0.25 & 0.48 & 0.69 & 0.41 & $\mathrm{~S}$ & - & 500 & rbf \\
c7 & 6 & 2 & 71 & 15 & 0.75 & 0.29 & 0.03 & 0.29 & 0.82 & 0.41 & $\mathrm{~S}$ & - & 1000 & rbf \\
c8 & 6 & 2 & 71 & 15 & 0.75 & 0.29 & 0.03 & 0.29 & 0.82 & 0.41 & $\mathrm{~S}$ & - & 500 & $\mathrm{rbf}$ \\
c9 & 6 & 2 & 71 & 15 & 0.75 & 0.29 & 0.03 & 0.29 & 0.82 & 0.41 & $\mathrm{~S}$ & - & 1000 & rbf \\
\hline
\end{tabular}

Após a aplicação da técnica PU, novos classificadores foram construídos e seus resultados (ver tabela 24) foram analisados. Para esses experimentos, o melhor modelo utilizou discretização EF e função sigmoidal com 1000 neurônios na camada escondida, 
atingindo $98 \%$ de acurácia, sem o uso de padronização. Resultados semelhantes foram obtidos em outros modelos, variando o número de neurônios da camada escondida entre 1000 e 1500. Além disso, observa-se que nenhum elemento positivo foi classificado incorretamente e que o número de falsos negativos caiu de sete para dois.

Tabela 24 - Resultados obtidos pelo classificador ELM com fator de custo 1.0 para o conjunto de dados AID721 após a aplicação do rotulador PU.

\begin{tabular}{|c|c|c|c|c|c|c|c|c|c|c|c|c|c|c|}
\hline id & VP & FP & VN & FN & Pre. & Rec. & $t_{f p}$ & $t_{v p}$ & Acc & $f_{1}$ & Scale & Disc & Neu. & Fa. \\
\hline c0 & 19 & 0 & 73 & 2 & 1.0 & 0.9 & 0.0 & 0.9 & 0.98 & 0.95 & - & EF & 1000 & sig \\
c1 & 19 & 0 & 73 & 2 & 1.0 & 0.9 & 0.0 & 0.9 & 0.98 & 0.95 & - & EF & 1500 & sig \\
c2 & 19 & 0 & 73 & 2 & 1.0 & 0.9 & 0.0 & 0.9 & 0.98 & 0.95 & - & EF & 1000 & sig \\
c3 & 19 & 0 & 73 & 2 & 1.0 & 0.9 & 0.0 & 0.9 & 0.98 & 0.95 & - & EF & 1500 & sig \\
c4 & 18 & 0 & 73 & 3 & 1.0 & 0.86 & 0.0 & 0.86 & 0.97 & 0.92 & $\mathrm{~S}$ & $\mathrm{EF}$ & 1500 & $\mathrm{sig}$ \\
c5 & 14 & 0 & 73 & 7 & 1.0 & 0.67 & 0.0 & 0.67 & 0.93 & 0.8 & - & $\mathrm{EF}$ & 500 & $\mathrm{rbf}$ \\
c6 & 14 & 0 & 73 & 7 & 1.0 & 0.67 & 0.0 & 0.67 & 0.93 & 0.8 & - & $\mathrm{EF}$ & 500 & $\mathrm{rbf}$ \\
c7 & 15 & 2 & 71 & 6 & 0.88 & 0.71 & 0.03 & 0.71 & 0.91 & 0.79 & - & $\mathrm{EW}$ & 1000 & sig \\
c8 & 13 & 0 & 73 & 8 & 1.0 & 0.62 & 0.0 & 0.62 & 0.91 & 0.76 & $\mathrm{~S}$ & $\mathrm{EF}$ & 1500 & $\mathrm{rbf}$ \\
c9 & 13 & 2 & 71 & 8 & 0.87 & 0.62 & 0.03 & 0.62 & 0.89 & 0.72 & $\mathrm{~S}$ & $\mathrm{EF}$ & 1000 & $\mathrm{sig}$ \\
\hline
\end{tabular}

Os desempenhos dos melhores modelos de classificação (antes e após aplicação da técnica PU) também podem ser comparados por meio da análise do espaço ROC, conforme apresenta a figura 13. Nota-se que, após a estimação dos rótulos via PU, os pontos relativos aos classificadores avaliados moveram-se de áreas pouco favorecidas para áreas no espaço ROC associadas a melhores desempenhos.

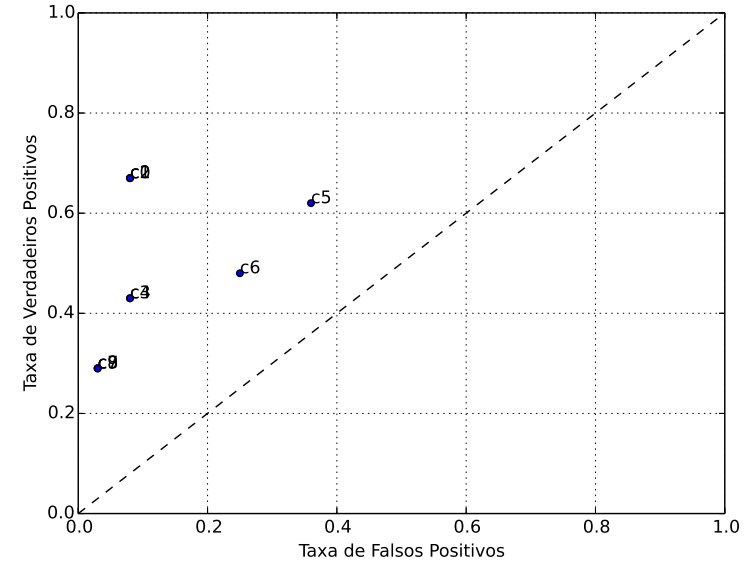

(a)

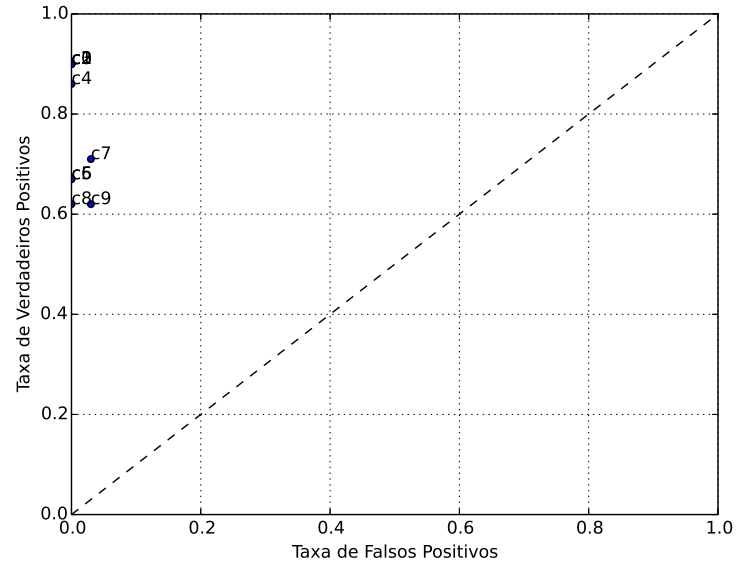

(b)

Figura 13 - Análise ROC para classificadores com fator de custo $C_{i j}=1.0$ para conjunto de dados 721. (a) Para dados com rotulação original (b) Para dados com rotulação após PU. 
Cenário 4: Análise de classificadores ELM com fator de custo $C_{i j}=1.0+\frac{1}{\text { total }_{p}+\text { total }_{n}}$

Os resultados alcançados pelo classificador construído para os dados com rotulação original são apresentados na tabela 25. O melhor resultado (acurácia igual a 65\%) foi obtido para os dados com padronização, com discretização EW e modelo ELM com 500 neurônios e função de ativação linear. Tal resultado é considerado insatisfatório, uma vez que encontra-se abaixo do erro majoritário do classificador, que igual a 77,66\%.

Tabela 25 - Resultados obtidos pelo classificador ELM com fator de custo $C_{i j}=1.0+$ $\frac{1}{\text { total }_{p}+\text { total }_{n}}$ para o conjunto de dados AID721 antes da aplicação do rotulador PU.

\begin{tabular}{|c|c|c|c|c|c|c|c|c|c|c|c|c|c|c|}
\hline id & VP & FP & VN & FN & Pre. & Rec. & $t_{f p}$ & $t_{v p}$ & Acc & $f_{1}$ & Scale & Disc & Neu. & Fa. \\
\hline c0 & 15 & 27 & 46 & 6 & 0.36 & 0.71 & 0.37 & 0.71 & 0.65 & 0.48 & $\mathrm{~S}$ & $\mathrm{EW}$ & 500 & lin \\
c1 & 18 & 43 & 30 & 3 & 0.3 & 0.86 & 0.59 & 0.86 & 0.51 & 0.44 & $\mathrm{~S}$ & - & 1000 & lin \\
c2 & 13 & 25 & 48 & 8 & 0.34 & 0.62 & 0.34 & 0.62 & 0.65 & 0.44 & - & $\mathrm{EF}$ & 1500 & lin \\
c3 & 18 & 43 & 30 & 3 & 0.3 & 0.86 & 0.59 & 0.86 & 0.51 & 0.44 & $\mathrm{~S}$ & - & 1000 & lin \\
c4 & 13 & 25 & 48 & 8 & 0.34 & 0.62 & 0.34 & 0.62 & 0.65 & 0.44 & - & $\mathrm{EF}$ & 1500 & lin \\
c5 & 13 & 25 & 48 & 8 & 0.34 & 0.62 & 0.34 & 0.62 & 0.65 & 0.44 & $\mathrm{~S}$ & $\mathrm{EW}$ & 1500 & lin \\
c6 & 12 & 25 & 48 & 9 & 0.32 & 0.57 & 0.34 & 0.57 & 0.64 & 0.41 & - & - & 500 & lin \\
c7 & 12 & 25 & 48 & 9 & 0.32 & 0.57 & 0.34 & 0.57 & 0.64 & 0.41 & $\mathrm{~S}$ & $\mathrm{EF}$ & 1000 & lin \\
c8 & 12 & 25 & 48 & 9 & 0.32 & 0.57 & 0.34 & 0.57 & 0.64 & 0.41 & - & $\mathrm{EW}$ & 1500 & lin \\
c9 & 12 & 25 & 48 & 9 & 0.32 & 0.57 & 0.34 & 0.57 & 0.64 & 0.41 & - & $\mathrm{EW}$ & 1500 & lin \\
\hline
\end{tabular}

Após a aplicação da técnica PU, novos classificadores foram construídos e seus resultados (ver tabela 26) foram analisados. Para esses experimentos, o melhor modelo utilizou discretização EF, sem padronização e função RBF com 1000 neurônios na camada escondida, atingindo $97 \%$ de acurácia. Além disso, observa-se que o número de falsos positivos caiu de 27 para um e que o número de falsos negativos caiu de seis para dois.

Tabela 26 - Resultados obtidos pelo classificador ELM com risco $C_{i j}=1.0+\frac{1}{\text { total }_{p}+\text { total }_{n}}$ para o conjunto de dados AID721 após a aplicação do rotulador PU.

\begin{tabular}{|c|c|c|c|c|c|c|c|c|c|c|c|c|c|c|}
\hline id & VP & FP & VN & FN & Pre. & Rec. & $t_{f p}$ & $t_{v p}$ & Acc & $f_{1}$ & Scale & Disc & Neu. & Fa. \\
\hline c0 & 19 & 1 & 72 & 2 & 0.95 & 0.9 & 0.01 & 0.9 & 0.97 & 0.93 & - & EF & 1500 & rbf \\
c1 & 19 & 1 & 72 & 2 & 0.95 & 0.9 & 0.01 & 0.9 & 0.97 & 0.93 & - & EF & 1500 & rbf \\
c2 & 18 & 0 & 73 & 3 & 1.0 & 0.86 & 0.0 & 0.86 & 0.97 & 0.92 & - & EF & 1000 & sig \\
c3 & 18 & 0 & 73 & 3 & 1.0 & 0.86 & 0.0 & 0.86 & 0.97 & 0.92 & - & EF & 1500 & sig \\
c4 & 18 & 0 & 73 & 3 & 1.0 & 0.86 & 0.0 & 0.86 & 0.97 & 0.92 & - & EF & 500 & sig \\
c5 & 18 & 0 & 73 & 3 & 1.0 & 0.86 & 0.0 & 0.86 & 0.97 & 0.92 & - & EF & 1000 & sig \\
c6 & 18 & 0 & 73 & 3 & 1.0 & 0.86 & 0.0 & 0.86 & 0.97 & 0.92 & - & EF & 1500 & sig \\
c7 & 18 & 0 & 73 & 3 & 1.0 & 0.86 & 0.0 & 0.86 & 0.97 & 0.92 & - & EF & 500 & sig \\
c8 & 18 & 2 & 71 & 3 & 0.9 & 0.86 & 0.03 & 0.86 & 0.95 & 0.88 & - & EF & 500 & rbf \\
c9 & 18 & 2 & 71 & 3 & 0.9 & 0.86 & 0.03 & 0.86 & 0.95 & 0.88 & - & EF & 500 & rbf \\
\hline
\end{tabular}


Avaliando o desempenho dos classificadores produzidos sob o espaço ROC (ver figura 14), é possível observar que o melhor classificador, aqui identificado com c0, é posicionado em uma área do gráfico dita conservadora. Mais especificamente, o melhor classificador necessita de mais evidências para classificar uma instância como positiva, produzindo assim uma taxa de falso positivo baixa.

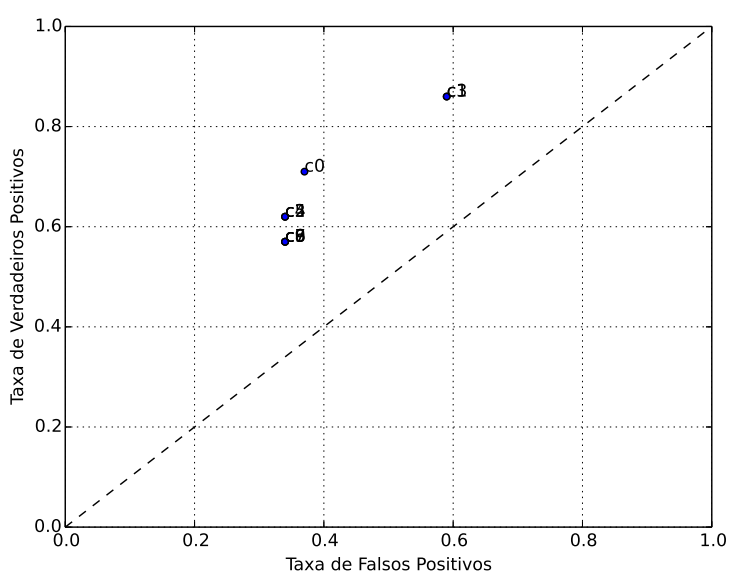

(a)

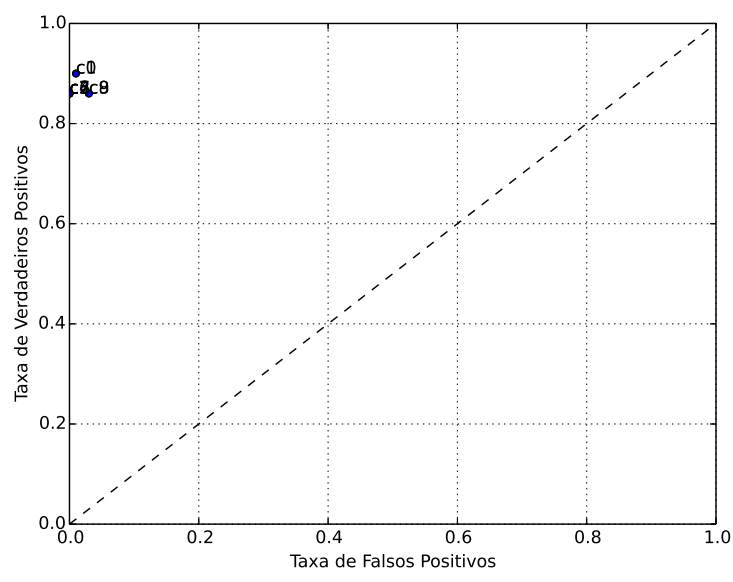

(b)

Figura 14 - (a)Modelos produzidos utilizando $C_{i j}=1.0+\frac{\text { total }_{p}}{\text { total }_{n}}$ para conjunto de dados AID721. (b)Modelos utilizando $C_{i j}=1.0+\frac{\text { total }_{n}}{\text { total }_{n}}$ para conjunto de dados AID721, com PU.

Em uma análise geral para o conjunto de dados AID721, somente o custo $C_{i j}=1.0$ $+\frac{1}{\text { total }_{p}+\text { total }_{n}}$, apresentou modelos selecionados com função RBF. Com discretização EF e 1500 neurônios na camada escondida, o modelo atingiu $97 \%$ de acurácia, mas o número de falsos positivos ficou acima de zero.

Para este conjunto de dados com erro majoritário de 77,66\%, um dos destaques foi a obtenção de $98 \%$ de acerto, sendo que utilizando o custo $C_{i j}=1.0$, e 1000 neurônios com funções sigmoidais na camada escondida, foi possível eliminar a ocorrência de elementos positivos identificados incorretamente.

\subsubsection{Experimentos de rotulação via PU - Conjunto de dados AID644}

A tabela 27 apresenta a nova distribuição após o processo PU, considerando a rotulação original que possui 67 elementos positivos e 139 elementos negativos: 
Tabela 27 - Distribuições de classes obtidas após a execução do processo PU contemplando a variação dos custos para o conjunto de dados AID644.

\begin{tabular}{|c|c|c|c|c|}
\hline Conjunto de dados & $\frac{1}{2}$ & $0.5+\frac{\text { total }_{p}}{\text { total }_{n}}$ & 1.0 & $1.0+\frac{\text { total }_{p}}{\text { total }_{n}}$ \\
\hline Bin. e Cont. + EW & $67 / 139$ & $80 / 123$ & $83 / 123$ & $83 / 123$ \\
\hline Cont. + Scale & $199 / 7$ & $78 / 128$ & $67 / 139$ & $67 / 139$ \\
\hline Bin. & $67 / 139$ & $82 / 124$ & $72 / 134$ & $78 / 128$ \\
\hline Bin. e Cont. & $191 / 15$ & $74 / 132$ & $76 / 130$ & $76 / 130$ \\
\hline Cont. & $195 / 11$ & $70 / 136$ & $87 / 119$ & $74 / 132$ \\
\hline Bin. e Cont. + Scale & $57 / 305$ & $60 / 302$ & $60 / 302$ & $62 / 300$ \\
\hline Cont. + Scale & $57 / 305$ & $102 / 260$ & $58 / 304$ & $58 / 304$ \\
\hline Bin. e Con. + EF & $181 / 25$ & $79 / 127$ & $96 / 110$ & $79 / 127$ \\
\hline Bin. e Cont. + Scale + EF & $67 / 139$ & $69 / 137$ & $81 / 125$ & $70 / 136$ \\
\hline Cont. + EF & $186 / 20$ & $73 / 133$ & $71 / 135$ & $72 / 134$ \\
\hline Cont. + Scale + EW & $156 / 50$ & $68 / 138$ & $69 / 117$ & $80 / 126$ \\
\hline Cont. + Scale + EF & $156 / 50$ & $77 / 129$ & $68 / 138$ & $67 / 139$ \\
\hline Bin. + Cont. + Scale + EW & $194 / 12$ & $83 / 123$ & $82 / 124$ & $78 / 128$ \\
\hline Cont. + EW & $184 / 22$ & $68 / 138$ & $68 / 138$ & $70 / 136$ \\
\hline \hline
\end{tabular}

Em relação aos resultados obtidos percebe-se que no que diz respeito ao custo $C_{i j}=1.0+\frac{t_{\text {otal }}}{\text { total }_{n}}$, as alterações são mais coerentes, sendo que os conjuntos que utilizaram padronização em seu pré-processamento se mantiveram próximos de sua distribuição original.

\subsubsection{Experimentos de classificação via ELM - Conjunto de dados AID644}

Nos próximos experimentos, classificadores ELM serão construídos para o conjunto de dados AID644, levando em conta sua rotulação original e as rotulações estimadas pelo método PU. Objetiva-se, com essa análise, avaliar o quanto esses diferentes cenários afetam o desempenho final do modelo de classificação.

Cenário 1: Análise de classificadores ELM com fator de custo $C_{i j}=\frac{1}{\text { total }_{p}+\text { total }_{n}}$

Os resultados alcançados pelo classificador construído para os dados com rotulação original são apresentados na tabela 16. O melhor resultado (acurácia igual a 77\%) foi obtido para os dados com padronização, com discretização e modelo ELM com 1000 neurônios e função de ativação sigmoidal. 
Tabela 28 - Resultados obtidos pelo classificador ELM com fator de custo $C_{i j}=\frac{1}{\text { total }_{p}+\text { total }_{n}}$ para o conjunto de dados AID644 antes da aplicação do rotulador PU.

\begin{tabular}{|c|c|c|c|c|c|c|c|c|c|c|c|c|c|c|}
\hline id & VP & FP & VN & FN & Pre. & Rec. & $t_{f p}$ & $t_{v p}$ & Acc & $f_{1}$ & Scale & Disc & Neu. & Fa. \\
\hline c0 & 66 & 46 & 93 & 1 & 0.59 & 0.99 & 0.33 & 0.99 & 0.77 & 0.74 & $\mathrm{~S}$ & $\mathrm{EW}$ & 1000 & sig \\
c1 & 65 & 94 & 45 & 2 & 0.41 & 0.97 & 0.68 & 0.97 & 0.53 & 0.58 & - & $\mathrm{EW}$ & 1000 & sig \\
c2 & 66 & 94 & 45 & 1 & 0.41 & 0.99 & 0.68 & 0.99 & 0.54 & 0.58 & - & $\mathrm{EW}$ & 1500 & sig \\
c3 & 66 & 94 & 45 & 1 & 0.41 & 0.99 & 0.68 & 0.99 & 0.54 & 0.58 & - & $\mathrm{EW}$ & 500 & sig \\
c4 & 63 & 95 & 44 & 4 & 0.4 & 0.94 & 0.68 & 0.94 & 0.52 & 0.56 & $\mathrm{~S}$ & $\mathrm{EF}$ & 1500 & lin \\
c5 & 60 & 97 & 42 & 7 & 0.38 & 0.9 & 0.7 & 0.9 & 0.5 & 0.54 & - & - & 1000 & lin \\
c6 & 60 & 95 & 44 & 7 & 0.39 & 0.9 & 0.68 & 0.9 & 0.5 & 0.54 & - & - & 1500 & lin \\
c7 & 60 & 94 & 45 & 7 & 0.39 & 0.9 & 0.68 & 0.9 & 0.51 & 0.54 & - & EF & 1000 & lin \\
c8 & 60 & 94 & 45 & 7 & 0.39 & 0.9 & 0.68 & 0.9 & 0.51 & 0.54 & - & EF & 1000 & lin \\
c9 & 57 & 92 & 47 & 10 & 0.38 & 0.85 & 0.66 & 0.85 & 0.5 & 0.53 & $\mathrm{~S}$ & - & 1500 & lin \\
\hline
\end{tabular}

Após a aplicação da técnica PU, novos classificadores foram construídos e seus resultados (ver tabela 29) foram analisados. Para esses experimentos, o melhor modelo utilizou discretização EF, padronização e função sigmoidal com 1000 neurônios na camada escondida, atingindo $100 \%$ de acurácia. Comportamento similar ocorreu em modelos que possuem quantidade menor de neurônios, independente do uso de padronizaçã. Vale destacar que a dicretização EF está presente na maioria dos modelos selecionados.

Tabela 29 - Resultados obtidos pelo classificador ELM com risco $C_{i j}=\frac{1}{\text { total }_{p}+\text { total }_{n}}$ para o conjunto de dados AID644 após a aplicação do rotulador PU.

\begin{tabular}{|c|c|c|c|c|c|c|c|c|c|c|c|c|c|c|}
\hline id & VP & FP & VN & FN & Pre. & Rec. & $t_{f p}$ & $t_{v p}$ & Acc & $f_{1}$ & Scale & Disc & Neu. & Fa. \\
\hline c0 & 66 & 0 & 139 & 1 & 1.0 & 0.99 & 0.0 & 0.99 & 1.0 & 0.99 & $\mathrm{~S}$ & EF & 1000 & sig \\
c1 & 66 & 0 & 139 & 1 & 1.0 & 0.99 & 0.0 & 0.99 & 1.0 & 0.99 & $\mathrm{~S}$ & EF & 500 & sig \\
c2 & 66 & 0 & 139 & 1 & 1.0 & 0.99 & 0.0 & 0.99 & 1.0 & 0.99 & - & EF & 1000 & sig \\
c3 & 66 & 0 & 139 & 1 & 1.0 & 0.99 & 0.0 & 0.99 & 1.0 & 0.99 & - & EF & 1500 & sig \\
c4 & 66 & 0 & 139 & 1 & 1.0 & 0.99 & 0.0 & 0.99 & 1.0 & 0.99 & - & EF & 1000 & sig \\
c5 & 66 & 0 & 139 & 1 & 1.0 & 0.99 & 0.0 & 0.99 & 1.0 & 0.99 & - & EF & 1500 & sig \\
c6 & 65 & 0 & 139 & 2 & 1.0 & 0.97 & 0.0 & 0.97 & 0.99 & 0.98 & $\mathrm{~S}$ & EF & 1500 & sig \\
c7 & 65 & 3 & 136 & 2 & 0.96 & 0.97 & 0.02 & 0.97 & 0.98 & 0.96 & $\mathrm{~S}$ & EW & 500 & sig \\
c8 & 52 & 4 & 135 & 15 & 0.93 & 0.78 & 0.03 & 0.78 & 0.91 & 0.85 & - & EF & 500 & sig \\
c9 & 52 & 4 & 135 & 15 & 0.93 & 0.78 & 0.03 & 0.78 & 0.91 & 0.85 & - & EF & 500 & sig \\
\hline
\end{tabular}

O desempenho dos melhores modelos também pode ser observado por meio da análise no espaço ROC, conforme a figura . Nota-se que, após a estimação dos rótulos via PU, os pontos relativos aos classificadores avaliados moveram-se de áreas pouco favorecidas para áreas no espaço ROC associadas a melhores desempenhos. 


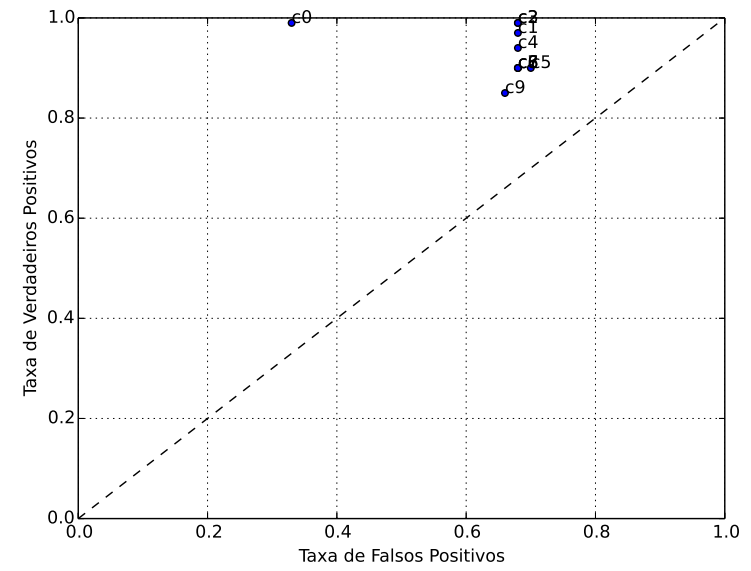

(a)

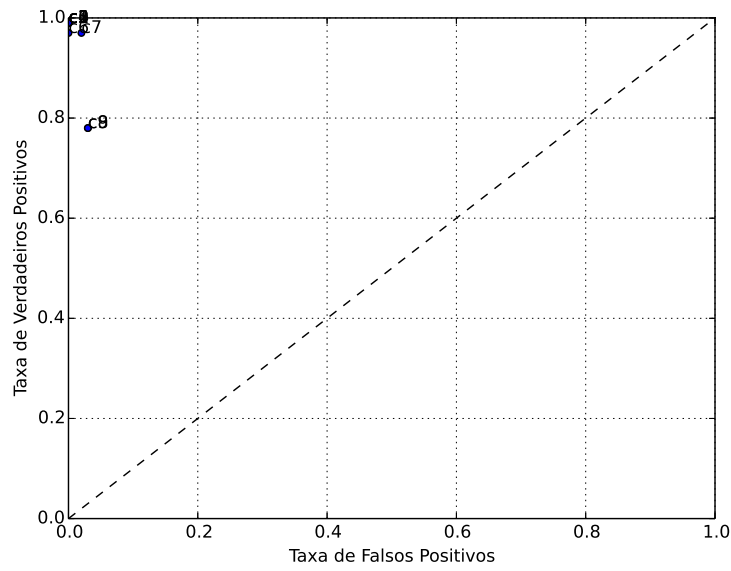

(b)

Figura 15 - (a)Modelos produzidos utilizando $C_{i j}=\frac{1}{\text { total }_{p}+\text { total }_{n}}$ para o conjunto de dados AID644. (b)Modelos utilizando $C_{i j}=\frac{1}{\text { total }_{p}+\text { total }_{n}}$ para o conjunto de dados AID644, com PU.

Cenário 2: Análise de classificadores ELM com fator de custo $C_{i j}=0.5+\frac{1}{\text { total }_{p}+\text { total }_{n}}$

Os resultados alcançados pelo classificador construído para os dados com rotulação original são apresentados na tabela 16. O melhor modelo apresentou acurácia igual a 56\%, abaixo do erro majoritário, que é de $67,48 \%$.

Tabela 30 - Resultados obtidos pelo classificador ELM com risco $C_{i j}=0.5+\frac{1}{\text { total }_{p}+\text { total }_{n}}$ para o conjunto de dados AID644 antes da aplicação do rotulador PU.

\begin{tabular}{|c|c|c|c|c|c|c|c|c|c|c|c|c|c|c|}
\hline id & VP & FP & VN & FN & Pre. & Rec. & $t_{f p}$ & $t_{v p}$ & Acc & $f_{1}$ & Scale & Disc & Neu. & Fa. \\
\hline c0 & 61 & 84 & 55 & 6 & 0.42 & 0.91 & 0.6 & 0.91 & 0.56 & 0.58 & - & EW & 1500 & lin \\
c1 & 61 & 84 & 55 & 6 & 0.42 & 0.91 & 0.6 & 0.91 & 0.56 & 0.58 & - & EW & 1500 & lin \\
c2 & 60 & 96 & 43 & 7 & 0.38 & 0.9 & 0.69 & 0.9 & 0.5 & 0.54 & - & EF & 1500 & lin \\
c3 & 61 & 96 & 43 & 6 & 0.39 & 0.91 & 0.69 & 0.91 & 0.5 & 0.54 & - & EF & 500 & lin \\
c4 & 60 & 96 & 43 & 7 & 0.38 & 0.9 & 0.69 & 0.9 & 0.5 & 0.54 & - & EF & 1500 & lin \\
c5 & 61 & 96 & 43 & 6 & 0.39 & 0.91 & 0.69 & 0.91 & 0.5 & 0.54 & - & EF & 500 & lin \\
c6 & 59 & 95 & 44 & 8 & 0.38 & 0.88 & 0.68 & 0.88 & 0.5 & 0.53 & $\mathrm{~S}$ & $\mathrm{EW}$ & 1500 & lin \\
c7 & 54 & 87 & 52 & 13 & 0.38 & 0.81 & 0.63 & 0.81 & 0.51 & 0.52 & - & - & 500 & lin \\
c8 & 56 & 97 & 42 & 11 & 0.37 & 0.84 & 0.7 & 0.84 & 0.48 & 0.51 & - & - & 1500 & lin \\
c9 & 54 & 95 & 44 & 13 & 0.36 & 0.81 & 0.68 & 0.81 & 0.48 & 0.5 & $\mathrm{~S}$ & $\mathrm{EF}$ & 1000 & lin \\
\hline
\end{tabular}

Para os experimentos após aplicação de PU, o melhor resultado foi para os dados com discretização EF e 1500 neurônios com função sigmoidal. Esta combinação produziu aproximadamente 100\% de acurácia sem o uso de padronização. Outro modelo, obteve 91\% de acurácia, mesmo sem o uso de padronização ou técnica de discretização. 
Tabela 31 - Resultados obtidos pelo classificador ELM com risco $C_{i j}=0.5+\frac{1}{\text { total }_{p}+\text { total }_{n}}$ para o conjunto de dados AID644 após a aplicação do rotulador PU.

\begin{tabular}{|c|c|c|c|c|c|c|c|c|c|c|c|c|c|c|}
\hline id & VP & FP & VN & FN & Pre. & Rec. & $t_{f p}$ & $t_{v p}$ & Acc & $f_{1}$ & Scale & Disc & Neu. & Fa. \\
\hline c0 & 66 & 0 & 139 & 1 & 1.0 & 0.99 & 0.0 & 0.99 & 1.0 & 0.99 & - & EF & 1500 & sig \\
c1 & 66 & 0 & 139 & 1 & 1.0 & 0.99 & 0.0 & 0.99 & 1.0 & 0.99 & - & EF & 1500 & sig \\
c2 & 63 & 9 & 130 & 4 & 0.88 & 0.94 & 0.06 & 0.94 & 0.94 & 0.91 & - & - & 1000 & lin \\
c3 & 58 & 9 & 130 & 9 & 0.87 & 0.87 & 0.06 & 0.87 & 0.91 & 0.87 & - & - & 1500 & lin \\
c4 & 49 & 3 & 136 & 18 & 0.94 & 0.73 & 0.02 & 0.73 & 0.9 & 0.82 & $\mathrm{~S}$ & $\mathrm{EF}$ & 1000 & rbf \\
c5 & 48 & 3 & 136 & 19 & 0.94 & 0.72 & 0.02 & 0.72 & 0.89 & 0.81 & - & EF & 1000 & sig \\
c6 & 48 & 3 & 136 & 19 & 0.94 & 0.72 & 0.02 & 0.72 & 0.89 & 0.81 & - & EF & 1000 & sig \\
c7 & 47 & 3 & 136 & 20 & 0.94 & 0.7 & 0.02 & 0.7 & 0.89 & 0.8 & $\mathrm{~S}$ & $\mathrm{EF}$ & 1500 & sig \\
c8 & 44 & 1 & 138 & 23 & 0.98 & 0.66 & 0.01 & 0.66 & 0.88 & 0.79 & $\mathrm{~S}$ & $\mathrm{EF}$ & 1000 & sig \\
c9 & 44 & 0 & 139 & 23 & 1.0 & 0.66 & 0.0 & 0.66 & 0.89 & 0.79 & $\mathrm{~S}$ & $\mathrm{EF}$ & 500 & sig \\
\hline
\end{tabular}

O desempenho dos melhores modelos também pode ser observado por meio da análise no espaço ROC, conforme a figura 16. Nota-se que, após a estimação dos rótulos via PU, os pontos relativos aos classificadores avaliados moveram-se de áreas pouco favorecidas para áreas no espaço ROC associadas a melhores desempenhos.

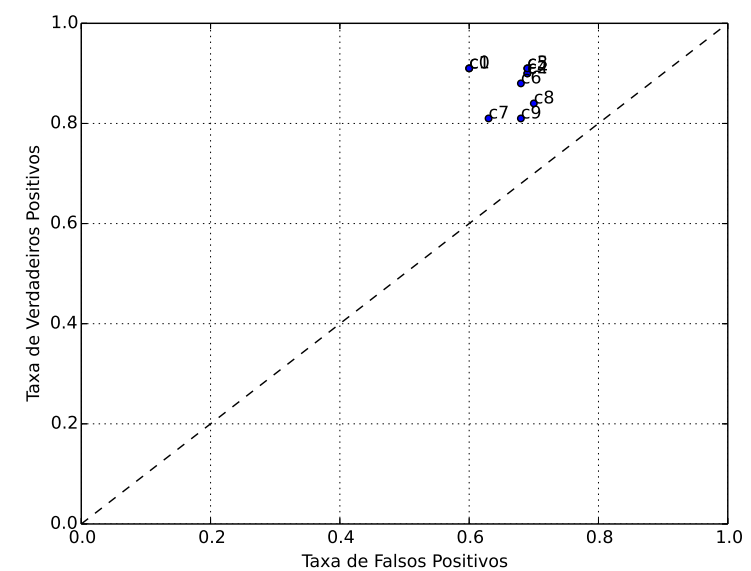

(a)

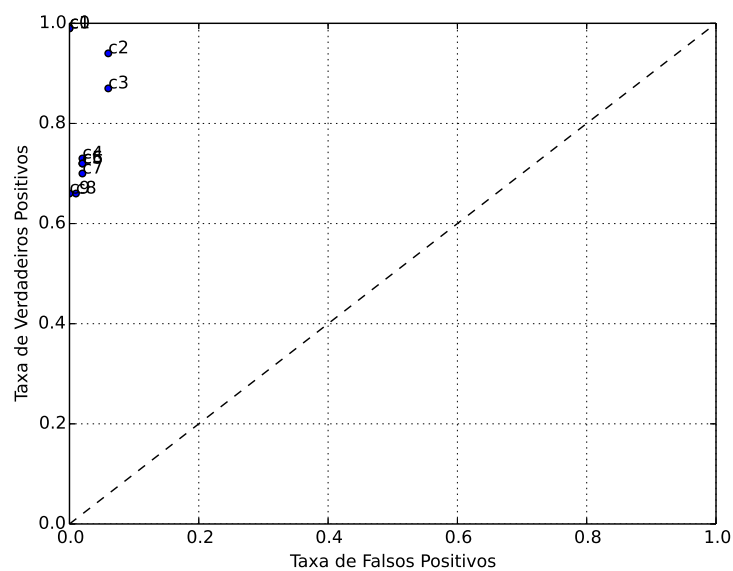

(b)

Figura 16 - (a) Modelos produzidos utilizando $C_{i j}=0.5+\frac{\text { total }_{p}}{\text { total }_{n}}$ para o conjunto de dados AID644. (b)Modelos utilizando $C_{i j}=0.5+\frac{\text { total }_{p}}{\text { total }_{n}}$ para o conjunto de dados AID644, com PU.

Cenário 3: Análise de classificadores ELM com fator de custo $C_{i j}=1.0$

Os resultados alcançados pelo classificador construído para os dados com rotulação original são apresentados na tabela 16. O melhor modelo apresentou acurácia igual a 70\%, próximo do erro majoritário, que é de $67,48 \%$. 
Tabela 32 - Resultados obtidos pelo classificador ELM com risco 1.0 para o conjunto de dados AID644 antes da aplicação do rotulador PU.

\begin{tabular}{|c|c|c|c|c|c|c|c|c|c|c|c|c|c|c|}
\hline id & VP & FP & VN & FN & Pre. & Rec. & $t_{f p}$ & $t_{v p}$ & Acc & $f_{1}$ & Scale & Disc & Neu. & Fa. \\
\hline c0 & 60 & 55 & 84 & 7 & 0.52 & 0.9 & 0.4 & 0.9 & 0.7 & 0.66 & $\mathrm{~S}$ & - & 1000 & lin \\
c1 & 60 & 55 & 84 & 7 & 0.52 & 0.9 & 0.4 & 0.9 & 0.7 & 0.66 & $\mathrm{~S}$ & - & 500 & lin \\
c2 & 60 & 55 & 84 & 7 & 0.52 & 0.9 & 0.4 & 0.9 & 0.7 & 0.66 & $\mathrm{~S}$ & - & 1000 & lin \\
c3 & 60 & 55 & 84 & 7 & 0.52 & 0.9 & 0.4 & 0.9 & 0.7 & 0.66 & $\mathrm{~S}$ & - & 500 & lin \\
c4 & 60 & 58 & 81 & 7 & 0.51 & 0.9 & 0.42 & 0.9 & 0.68 & 0.65 & $\mathrm{~S}$ & - & 1500 & lin \\
c5 & 60 & 58 & 81 & 7 & 0.51 & 0.9 & 0.42 & 0.9 & 0.68 & 0.65 & $\mathrm{~S}$ & - & 1500 & lin \\
c6 & 63 & 94 & 45 & 4 & 0.4 & 0.94 & 0.68 & 0.94 & 0.52 & 0.56 & - & EF & 500 & lin \\
c7 & 63 & 94 & 45 & 4 & 0.4 & 0.94 & 0.68 & 0.94 & 0.52 & 0.56 & - & EF & 500 & lin \\
c8 & 42 & 44 & 95 & 25 & 0.49 & 0.63 & 0.32 & 0.63 & 0.67 & 0.55 & $\mathrm{~S}$ & - & 1000 & sig \\
c9 & 42 & 44 & 95 & 25 & 0.49 & 0.63 & 0.32 & 0.63 & 0.67 & 0.55 & $\mathrm{~S}$ & - & 1000 & sig \\
\hline
\end{tabular}

Para os experimentos após aplicação de PU, o uso de padronização e função sigmoidal nos 1000 neurônios da camada escondida, produziram uma acurácia próxima de 100\%. Assim como nos demais experimentos aplicados neste conjunto de dados os melhores modelos possuem discretização EF.

Tabela 33 - Resultados obtidos pelo classificador ELM com risco 1.0 para o conjunto de dados AID644 após a aplicação do rotulador PU.

\begin{tabular}{|c|c|c|c|c|c|c|c|c|c|c|c|c|c|c|}
\hline id & VP & FP & VN & FN & Pre. & Rec. & $t_{f p}$ & $t_{v p}$ & Acc & $f_{1}$ & Scale & Disc & Neu. & Fa. \\
\hline c0 & 66 & 0 & 139 & 1 & 1.0 & 0.99 & 0.0 & 0.99 & 1.0 & 0.99 & - & EF & 1000 & sig \\
c1 & 66 & 0 & 139 & 1 & 1.0 & 0.99 & 0.0 & 0.99 & 1.0 & 0.99 & - & EF & 1000 & sig \\
c2 & 65 & 0 & 139 & 2 & 1.0 & 0.97 & 0.0 & 0.97 & 0.99 & 0.98 & $\mathrm{~S}$ & EF & 1500 & sig \\
c3 & 46 & 1 & 138 & 21 & 0.98 & 0.69 & 0.01 & 0.69 & 0.89 & 0.81 & $\mathrm{~S}$ & EF & 500 & sig \\
c4 & 45 & 1 & 138 & 22 & 0.98 & 0.67 & 0.01 & 0.67 & 0.89 & 0.8 & $\mathrm{~S}$ & $\mathrm{EF}$ & 1000 & $\mathrm{sig}$ \\
c5 & 44 & 1 & 138 & 23 & 0.98 & 0.66 & 0.01 & 0.66 & 0.88 & 0.79 & $\mathrm{~S}$ & $\mathrm{EF}$ & 1500 & $\mathrm{rbf}$ \\
c6 & 44 & 1 & 138 & 23 & 0.98 & 0.66 & 0.01 & 0.66 & 0.88 & 0.79 & $\mathrm{~S}$ & $\mathrm{EF}$ & 500 & $\mathrm{rbf}$ \\
c7 & 45 & 2 & 137 & 22 & 0.96 & 0.67 & 0.01 & 0.67 & 0.88 & 0.79 & - & $\mathrm{EF}$ & 1500 & $\mathrm{rbf}$ \\
c8 & 47 & 5 & 134 & 20 & 0.9 & 0.7 & 0.04 & 0.7 & 0.88 & 0.79 & - & $\mathrm{EF}$ & 1500 & sig \\
c9 & 45 & 2 & 137 & 22 & 0.96 & 0.67 & 0.01 & 0.67 & 0.88 & 0.79 & - & $\mathrm{EF}$ & 500 & sig \\
\hline
\end{tabular}

O desempenho dos melhores modelos também pode ser observado por meio da análise no espaço ROC, conforme a figura 17. Nota-se que, após a estimação dos rótulos via PU, os pontos relativos aos classificadores avaliados moveram-se de áreas pouco favorecidas para áreas no espaço ROC associadas a melhores desempenhos. Para esses experimentos, observa-se a prevalência de modelos menos conservadores, os quais efetuam a classificação das instâncias positivas com poucas evidências, fato este comprovado pelas altas taxas de falsos positivos. Por outro lado, após a estimação feita pelo processo PU, observa-se que 
todos os modelos selecionados possuem taxas de falso positivos baixa e se posicionam em uma área mais conservadorora do espaço ROC.

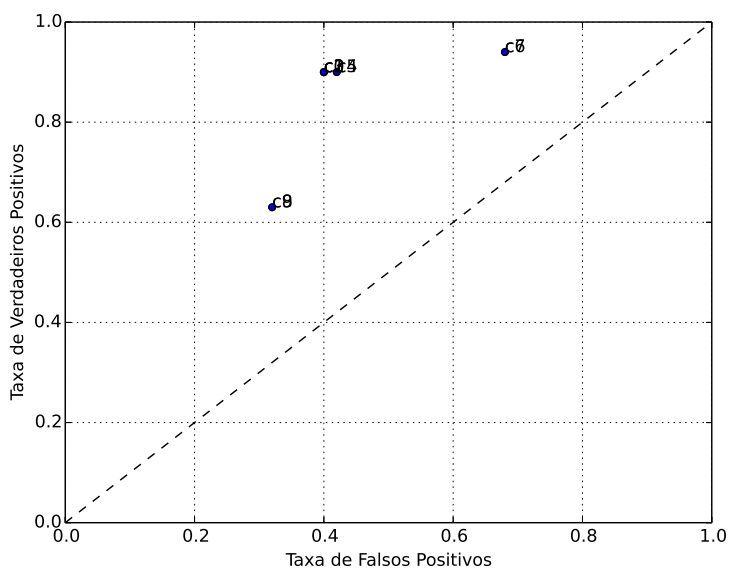

(a)

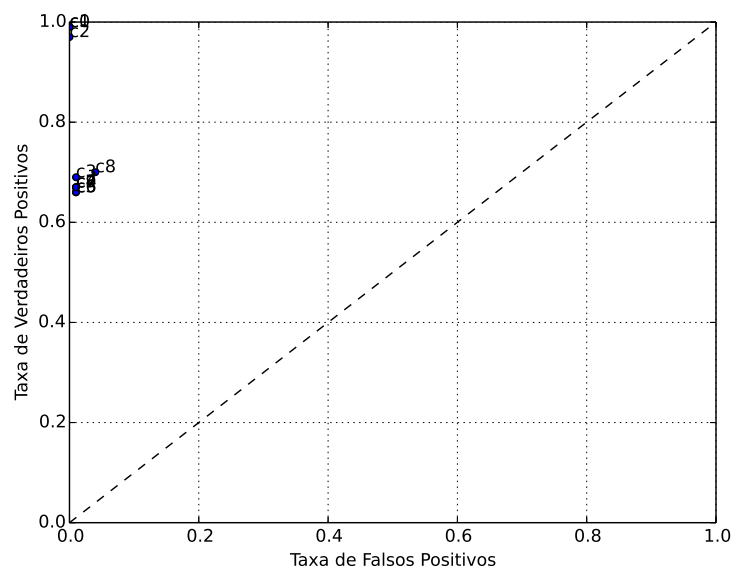

(b)

Figura 17 - (a)Modelos produzidos utilizando $C_{i j}=1.0$ para o conjunto de dados AID644.

(b)Modelos utilizando $C_{i j}=1.0$ para o conjunto de dados AID644, com PU.

Cenário 4: Análise de classificadores ELM com fator de custo $C_{i j}=1.0+\frac{1}{\text { total }_{p}+\text { total }_{n}}$

Os resultados alcançados pelo classificador construído para os dados com rotulação original são apresentados na tabela 16. O melhor modelo apresentou acurácia igual a 52\%, abaixo do erro majoritário, que é de $67,48 \%$.

Tabela 34 - Resultados obtidos pelo classificador ELM com risco $C_{i j}=1.0+\frac{1}{\text { total }_{p}+\text { total }_{n}}$ para o conjunto de dados AID644 antes da aplicação do rotulador PU.

\begin{tabular}{|c|c|c|c|c|c|c|c|c|c|c|c|c|c|l|}
\hline id & VP & FP & VN & FN & Pre. & Rec. & $t_{f p}$ & $t_{v p}$ & Acc & $f_{1}$ & Scale & Disc & Neu. & Fa. \\
\hline c0 & 63 & 94 & 45 & 4 & 0.4 & 0.94 & 0.68 & 0.94 & 0.52 & 0.56 & $\mathrm{~S}$ & $\mathrm{EF}$ & 500 & lin \\
c1 & 62 & 95 & 44 & 5 & 0.39 & 0.93 & 0.68 & 0.93 & 0.51 & 0.55 & $\mathrm{~S}$ & $\mathrm{EF}$ & 1000 & lin \\
c2 & 61 & 94 & 45 & 6 & 0.39 & 0.91 & 0.68 & 0.91 & 0.51 & 0.55 & - & $\mathrm{EW}$ & 1500 & lin \\
c3 & 61 & 94 & 45 & 6 & 0.39 & 0.91 & 0.68 & 0.91 & 0.51 & 0.55 & - & $\mathrm{EW}$ & 1500 & lin \\
c4 & 61 & 96 & 43 & 6 & 0.39 & 0.91 & 0.69 & 0.91 & 0.5 & 0.54 & $\mathrm{~S}$ & $\mathrm{EF}$ & 1000 & lin \\
c5 & 59 & 94 & 45 & 8 & 0.39 & 0.88 & 0.68 & 0.88 & 0.5 & 0.54 & $\mathrm{~S}$ & $\mathrm{EW}$ & 500 & lin \\
c6 & 60 & 96 & 43 & 7 & 0.38 & 0.9 & 0.69 & 0.9 & 0.5 & 0.54 & - & $\mathrm{EF}$ & 1500 & lin \\
c7 & 60 & 98 & 41 & 7 & 0.38 & 0.9 & 0.71 & 0.9 & 0.49 & 0.53 & $\mathrm{~S}$ & $\mathrm{EF}$ & 500 & lin \\
c8 & 59 & 101 & 38 & 8 & 0.37 & 0.88 & 0.73 & 0.88 & 0.47 & 0.52 & $\mathrm{~S}$ & $\mathrm{EF}$ & 1500 & lin \\
c9 & 58 & 96 & 43 & 9 & 0.38 & 0.87 & 0.69 & 0.87 & 0.49 & 0.52 & - & $\mathrm{EF}$ & 500 & lin \\
\hline
\end{tabular}

Para os experimentos após aplicação de PU, observa-se que apesar da existência de vários classificadores com acurácia próxima a 100\%, o modelo com melhor desempenho utiliza padronização e possui 1500 neurônios em sua camada escondida. Sem classificar 
nenhum elemento positivo de forma incorreta, o modelo utiliza também utiliza discretização EF em sua fase de pré-processamento.

Tabela 35 - Resultados obtidos pelo classificador ELM com risco $C_{i j}=1.0+\frac{1}{\text { total }_{p}+\text { total }_{n}}$ para o conjunto de dados AID644 após a aplicação do rotulador PU.

\begin{tabular}{|c|c|c|c|c|c|c|c|c|c|c|c|c|c|c|}
\hline id & VP & FP & VN & FN & Pre. & Rec. & $t_{f p}$ & $t_{v p}$ & Acc & $f_{1}$ & Scale & Disc & Neu. & Fa. \\
\hline c0 & 66 & 0 & 139 & 1 & 1.0 & 0.99 & 0.0 & 0.99 & 1.0 & 0.99 & $\mathrm{~S}$ & EF & 1500 & sig \\
c1 & 66 & 1 & 138 & 1 & 0.99 & 0.99 & 0.01 & 0.99 & 0.99 & 0.99 & $\mathrm{~S}$ & $\mathrm{EF}$ & 1000 & $\mathrm{sig}$ \\
c2 & 67 & 1 & 138 & 0 & 0.99 & 1.0 & 0.01 & 1.0 & 1.0 & 0.99 & - & $\mathrm{EF}$ & 1000 & $\mathrm{rbf}$ \\
c3 & 66 & 0 & 139 & 1 & 1.0 & 0.99 & 0.0 & 0.99 & 1.0 & 0.99 & - & $\mathrm{EF}$ & 1000 & sig \\
c4 & 66 & 0 & 139 & 1 & 1.0 & 0.99 & 0.0 & 0.99 & 1.0 & 0.99 & - & $\mathrm{EF}$ & 1500 & $\mathrm{sig}$ \\
c5 & 67 & 1 & 138 & 0 & 0.99 & 1.0 & 0.01 & 1.0 & 1.0 & 0.99 & - & $\mathrm{EF}$ & 1000 & $\mathrm{rbf}$ \\
c6 & 66 & 0 & 139 & 1 & 1.0 & 0.99 & 0.0 & 0.99 & 1.0 & 0.99 & - & $\mathrm{EF}$ & 1000 & sig \\
c7 & 66 & 0 & 139 & 1 & 1.0 & 0.99 & 0.0 & 0.99 & 1.0 & 0.99 & - & $\mathrm{EF}$ & 1500 & sig \\
c8 & 66 & 3 & 136 & 1 & 0.96 & 0.99 & 0.02 & 0.99 & 0.98 & 0.97 & $\mathrm{~S}$ & $\mathrm{EF}$ & 500 & sig \\
c9 & 50 & 5 & 134 & 17 & 0.91 & 0.75 & 0.04 & 0.75 & 0.89 & 0.82 & $\mathrm{~S}$ & $\mathrm{EF}$ & 500 & $\mathrm{rbf}$ \\
\hline
\end{tabular}

O desempenho dos melhores modelos também pode ser observado por meio da análise no espaço ROC, conforme a figura 18. Nota-se que, após a estimação dos rótulos via $\mathrm{PU}$, os pontos relativos aos classificadores avaliados moveram-se de áreas pouco favorecidas para áreas no espaço ROC associadas a melhores desempenhos. Avaliando o desempenho dos classificadores produzidos sob o espaço ROC, é possível observar que o melhor classificador, aqui identificado com c0, é posicionado em uma área do gráfico dita conservadora. Mais especificamente, o melhor classificador necessita de mais evidências para classificar uma instância como positiva, produzindo assim uma taxa de falso positivo baixa. 


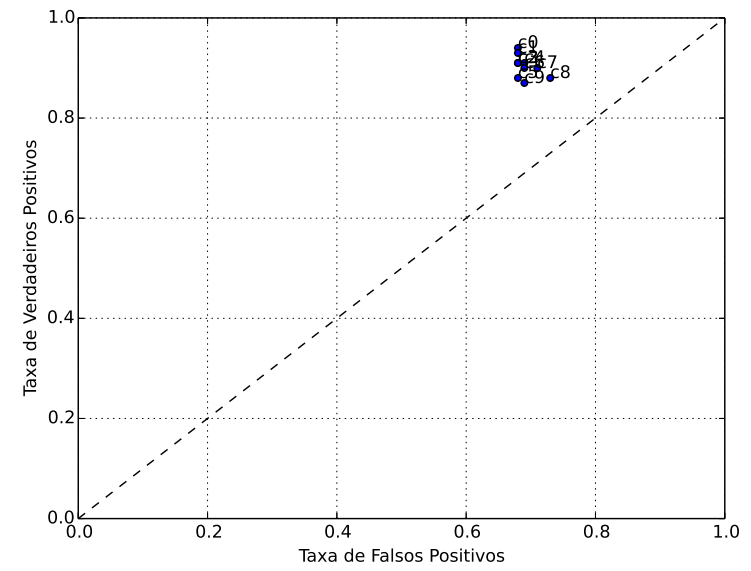

(a)

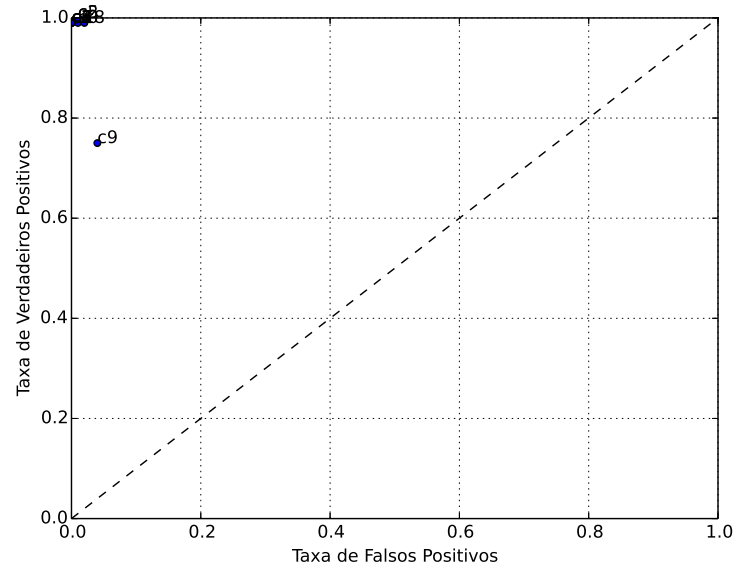

(b)

Figura 18 - (a)Modelos produzidos utilizando $C_{i j}=1.0+\frac{\text { total }_{p}}{\text { total }_{n}}$ para o conjunto de dados AID644. (b)Modelos utilizando $C_{i j}=1.0+\frac{\text { total }_{n}}{\text { total }_{n}}$ para o conjunto de dados AID644, com PU.

Em uma análise geral, para conjunto de dados AID644 o erro majoritário é de $67,47 \%$ e, para o custo $C_{i j}=1.0+\frac{t_{\text {total }}}{\text { totaln }_{n}}$, foi obtida uma acurácia próxima à $100 \%$. Tal experimento foi executado com dados padronizados, discretização utilizando a técnica EF e com 1000 neurônios com funções sigmoidais na camada oculta.

8.3.8 Experimentos de rotulação via PU - Conjunto de dados AID439

A tabela 36 apresenta as distribuições obtidas após o processo PU para o conjunto de dados AID439, considerando a rotulação original que possui 13 elementos positivos e 56 elementos negativos: 
Tabela 36 - Distribuições de classes obtidas após a execução do processo PU contemplando a variação dos custos para o conjunto de dados AID439.

\begin{tabular}{|c|c|c|c|c|}
\hline Conjunto de dados & $\frac{1}{2}$ & $0.5+\frac{\text { total }_{p}}{\text { total }_{n}}$ & 1.0 & $1.0+\frac{\text { total }_{p}}{\text { total }_{n}}$ \\
\hline Bin. e Cont. + EW & $47 / 22$ & $23 / 46$ & $18 / 51$ & $14 / 55$ \\
\hline Cont. + Scale & $13 / 56$ & $18 / 51$ & $20 / 49$ & $18 / 51$ \\
\hline Bin. & $52 / 17$ & $16 / 53$ & $14 / 55$ & $19 / 50$ \\
\hline Bin. e Cont. & $13 / 56$ & $17 / 52$ & $22 / 47$ & $37 / 32$ \\
\hline Cont. & $13 / 56$ & $24 / 45$ & $13 / 56$ & $35 / 34$ \\
\hline Bin. e Cont. + Scale & $57 / 305$ & $60 / 302$ & $60 / 302$ & $62 / 300$ \\
\hline Cont. + Scale & $57 / 305$ & $102 / 260$ & $58 / 304$ & $58 / 304$ \\
\hline Bin. e Con. + EF & $21 / 48$ & $16 / 53$ & $63 / 6$ & $23 / 46$ \\
\hline Bin. e Cont. + Scale + EF & $19 / 50$ & $14 / 55$ & $14 / 55$ & $14 / 55$ \\
\hline Cont. + EF & $13 / 56$ & $32 / 37$ & $18 / 51$ & $35 / 34$ \\
\hline Cont. + Scale + EW & $21 / 48$ & $16 / 53$ & $16 / 53$ & $30 / 39$ \\
\hline Cont. + Scale + EF & $18 / 51$ & $14 / 54$ & $14 / 55$ & $32 / 37$ \\
\hline Bin. + Cont. + Scale + EW & $23 / 46$ & $21 / 48$ & $13 / 56$ & $13 / 56$ \\
\hline Cont. + EW & $24 / 45$ & $31 / 38$ & $55 / 14$ & $28 / 41$ \\
\hline \hline
\end{tabular}

Nesses experimentos observa-se que, independente do custo utilizado, alguns dos novos conjuntos manteve sua rotulação inalterada. Percebe-se também, que algumas configurações que utilizam atributos binários juntamente com contínuos e atributos contínuos com padronização, apresentaram alteração significante da rotulação independente do custo utilizado. Contudo, para a utilização do custo 1.0, as alterações se mantiveram próximas às rotulações originais.

\subsubsection{Experimentos de classificação via ELM - Conjunto de dados AID439}

Nos próximos experimentos, classificadores ELM serão construídos para o conjunto de dados AID439, levando em conta sua rotulação original e as rotulações estimadas pelo método PU. Objetiva-se, com essa análise, avaliar o quanto esses diferentes cenários afetam o desempenho final do modelo de classificação.

Cenário 1: Análise de classificadores ELM com fator de custo $C_{i j}=\frac{1}{\text { total }_{p}+\text { total }_{n}}$

Os resultados alcançados pelo classificador construído para os dados com rotulação original são apresentados na tabela 37. O melhor modelo apresentou acurácia igual a 96\%. 
Tabela 37 - Resultados obtidos pelo classificador ELM com risco $C_{i j}=\frac{1}{\text { total }_{p}+\text { total }_{n}}$ para o conjunto de dados AID439 antes da aplicação do rotulador PU.

\begin{tabular}{|c|c|c|c|c|c|c|c|c|c|c|c|c|c|c|}
\hline id & VP & FP & VN & FN & Pre. & Rec. & $t_{f p}$ & $t_{v p}$ & Acc & $f_{1}$ & Scale & Disc & Neu. & Fa. \\
\hline c0 & 10 & 0 & 56 & 3 & 1.0 & 0.77 & 0.0 & 0.77 & 0.96 & 0.87 & - & EF & 1000 & lin \\
c1 & 8 & 1 & 55 & 5 & 0.89 & 0.62 & 0.02 & 0.62 & 0.91 & 0.73 & $\mathrm{~S}$ & - & 1000 & rbf \\
c2 & 8 & 1 & 55 & 5 & 0.89 & 0.62 & 0.02 & 0.62 & 0.91 & 0.73 & $\mathrm{~S}$ & - & 1500 & rbf \\
c3 & 8 & 1 & 55 & 5 & 0.89 & 0.62 & 0.02 & 0.62 & 0.91 & 0.73 & $\mathrm{~S}$ & - & 1500 & sig \\
c4 & 8 & 1 & 55 & 5 & 0.89 & 0.62 & 0.02 & 0.62 & 0.91 & 0.73 & $\mathrm{~S}$ & - & 500 & sig \\
c5 & 8 & 1 & 55 & 5 & 0.89 & 0.62 & 0.02 & 0.62 & 0.91 & 0.73 & $\mathrm{~S}$ & - & 1000 & $\mathrm{rbf}$ \\
c6 & 8 & 1 & 55 & 5 & 0.89 & 0.62 & 0.02 & 0.62 & 0.91 & 0.73 & $\mathrm{~S}$ & - & 1500 & rbf \\
c7 & 8 & 1 & 55 & 5 & 0.89 & 0.62 & 0.02 & 0.62 & 0.91 & 0.73 & $\mathrm{~S}$ & - & 1500 & sig \\
c8 & 8 & 1 & 55 & 5 & 0.89 & 0.62 & 0.02 & 0.62 & 0.91 & 0.73 & $\mathrm{~S}$ & - & 500 & sig \\
c9 & 7 & 2 & 54 & 6 & 0.78 & 0.54 & 0.04 & 0.54 & 0.88 & 0.64 & - & - & 1000 & sig \\
\hline
\end{tabular}

Para os experimentos após aplicação de PU, observa-se que apesar da existência de vários classificadores com acurácia próxima a 100\%, o modelo com melhor desempenho utiliza padronização e possui 1500 neurônios em sua camada escondida. Sem classificar nenhum elemento positivo de forma incorreta, o modelo utiliza também utiliza discretização EF em sua fase de pré-processamento.

Tabela 38 - Resultados obtidos pelo classificador ELM com risco $C_{i j}=\frac{1}{\text { total }_{p}+\text { total }_{n}}$ para o conjunto de dados AID439 após a aplicação do rotulador PU.

\begin{tabular}{|c|c|c|c|c|c|c|c|c|c|c|c|c|c|c|}
\hline id & VP & FP & VN & FN & Pre. & Rec. & $t_{f p}$ & $t_{v p}$ & Acc & $f_{1}$ & Scale & Disc & Neu. & Fa. \\
\hline c0 & 13 & 1 & 55 & 0 & 0.93 & 1.0 & 0.02 & 1.0 & 0.99 & 0.96 & $\mathrm{~S}$ & $\mathrm{EW}$ & 500 & lin \\
c1 & 12 & 1 & 55 & 1 & 0.92 & 0.92 & 0.02 & 0.92 & 0.97 & 0.92 & $\mathrm{~S}$ & $\mathrm{EW}$ & 1000 & lin \\
c2 & 10 & 0 & 56 & 3 & 1.0 & 0.77 & 0.0 & 0.77 & 0.96 & 0.87 & $\mathrm{~S}$ & $\mathrm{EW}$ & 1000 & $\mathrm{rbf}$ \\
c3 & 10 & 0 & 56 & 3 & 1.0 & 0.77 & 0.0 & 0.77 & 0.96 & 0.87 & $\mathrm{~S}$ & $\mathrm{EW}$ & 500 & sig \\
c4 & 10 & 0 & 56 & 3 & 1.0 & 0.77 & 0.0 & 0.77 & 0.96 & 0.87 & $\mathrm{~S}$ & $\mathrm{EF}$ & 1000 & sig \\
c5 & 10 & 0 & 56 & 3 & 1.0 & 0.77 & 0.0 & 0.77 & 0.96 & 0.87 & $\mathrm{~S}$ & $\mathrm{EF}$ & 500 & sig \\
c6 & 10 & 0 & 56 & 3 & 1.0 & 0.77 & 0.0 & 0.77 & 0.96 & 0.87 & $\mathrm{~S}$ & $\mathrm{EW}$ & 1500 & lin \\
c7 & 9 & 0 & 56 & 4 & 1.0 & 0.69 & 0.0 & 0.69 & 0.94 & 0.82 & $\mathrm{~S}$ & - & 1000 & lin \\
c8 & 9 & 0 & 56 & 4 & 1.0 & 0.69 & 0.0 & 0.69 & 0.94 & 0.82 & $\mathrm{~S}$ & $\mathrm{EF}$ & 1000 & rbf \\
c9 & 9 & 0 & 56 & 4 & 1.0 & 0.69 & 0.0 & 0.69 & 0.94 & 0.82 & $\mathrm{~S}$ & $\mathrm{EF}$ & 1500 & rbf \\
\hline
\end{tabular}

O desempenho dos melhores modelos também pode ser observado por meio da análise no espaço ROC, conforme a figura 19. Nota-se que, após a estimação dos rótulos via $\mathrm{PU}$, os pontos relativos aos classificadores avaliados moveram-se de áreas pouco favorecidas para áreas no espaço ROC associadas a melhores desempenhos. Nesses experimentos, observa-se que os modelos selecionados apresentaram baixa taxa de falsos positivos, mesmo sem a aplicação do método PU. Após a estimação da rotulação, além da redução da taxa de 
verdadeiros positivos, percebe-se também o crescimento da taxa de verdadeiros positivos, melhorando o desempenho geral.

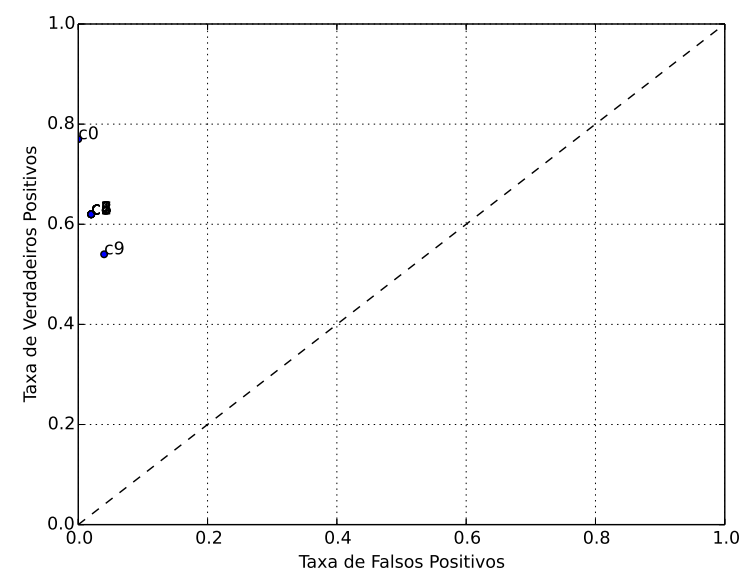

(a)

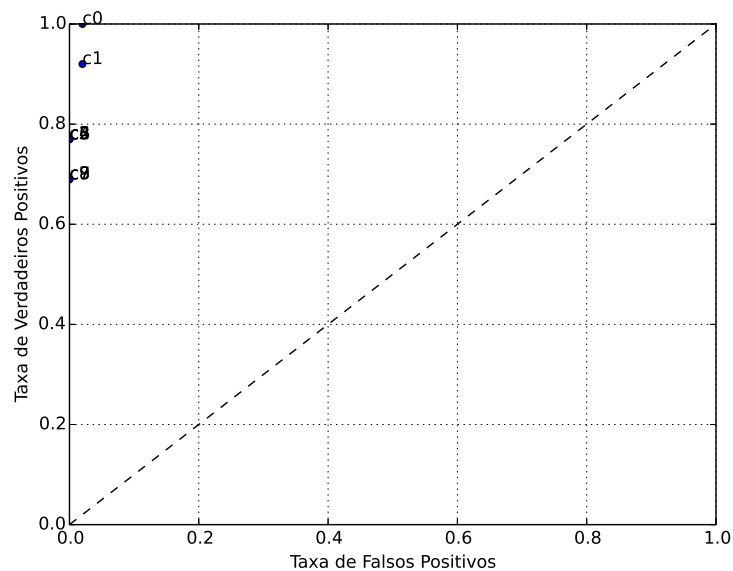

(b)

Figura 19 - (a)Modelos produzidos utilizando $C_{i j}=\frac{1}{\text { total }_{p}+\text { total }_{n}}$ para o conjunto de dados AID439. (b)Modelos utilizando $C_{i j}=\frac{1}{\text { total }_{p}+\text { total }_{n}}$ para o conjunto de dados AID439, aplicando PU.

Cenário 2: Análise de classificadores ELM com fator de custo $C_{i j}=0.5+\frac{1}{\text { total }_{p}+\text { total }_{n}}$

Os resultados alcançados pelo classificador construído para os dados com rotulação original são apresentados na tabela 39. O melhor modelo apresentou acurácia igual a 77\%, abaixo do erro majoritário, que é de 81,06\%.

Tabela 39 - Resultados obtidos pelo classificador ELM com risco $C_{i j}=0.5+\frac{1}{\text { total }_{p}+\text { total }_{n}}$ para o conjunto de dados AID439 antes da aplicação do rotulador PU.

\begin{tabular}{|c|c|c|c|c|c|c|c|c|c|c|c|c|c|c|}
\hline id & VP & FP & VN & FN & Pre. & Rec. & $t_{f p}$ & $t_{v p}$ & Acc & $f_{1}$ & Scale & Disc & Neu. & Fa. \\
c0 & 10 & 13 & 43 & 3 & 0.43 & 0.77 & 0.23 & 0.77 & 0.77 & 0.56 & $\mathrm{~S}$ & - & 1000 & rbf \\
c1 & 10 & 13 & 43 & 3 & 0.43 & 0.77 & 0.23 & 0.77 & 0.77 & 0.56 & $\mathrm{~S}$ & - & 1000 & $\mathrm{rbf}$ \\
$\mathrm{c} 2$ & 7 & 7 & 49 & 6 & 0.5 & 0.54 & 0.13 & 0.54 & 0.81 & 0.52 & - & $\mathrm{EW}$ & 1000 & $\mathrm{rbf}$ \\
$\mathrm{c} 3$ & 7 & 7 & 49 & 6 & 0.5 & 0.54 & 0.13 & 0.54 & 0.81 & 0.52 & - & $\mathrm{EW}$ & 1000 & $\mathrm{rbf}$ \\
$\mathrm{c} 4$ & 7 & 9 & 47 & 6 & 0.44 & 0.54 & 0.16 & 0.54 & 0.78 & 0.48 & - & - & 1000 & $\mathrm{sig}$ \\
$\mathrm{c} 5$ & 9 & 18 & 38 & 4 & 0.33 & 0.69 & 0.32 & 0.69 & 0.68 & 0.45 & - & $\mathrm{EW}$ & 500 & $\mathrm{rbf}$ \\
$\mathrm{c} 6$ & 9 & 18 & 38 & 4 & 0.33 & 0.69 & 0.32 & 0.69 & 0.68 & 0.45 & - & $\mathrm{EW}$ & 500 & $\mathrm{rbf}$ \\
$\mathrm{c} 7$ & 7 & 14 & 42 & 6 & 0.33 & 0.54 & 0.25 & 0.54 & 0.71 & 0.41 & $\mathrm{~S}$ & $\mathrm{EF}$ & 1500 & $\mathrm{sig}$ \\
$\mathrm{c} 8$ & 9 & 24 & 32 & 4 & 0.27 & 0.69 & 0.43 & 0.69 & 0.59 & 0.39 & - & - & 500 & $\mathrm{rbf}$ \\
$\mathrm{c} 9$ & 7 & 16 & 40 & 6 & 0.3 & 0.54 & 0.29 & 0.54 & 0.68 & 0.39 & - & $\mathrm{EW}$ & 1500 & $\mathrm{rbf}$ \\
\hline
\end{tabular}

Para os experimentos após aplicação de PU, observa-se que o melhor experimento foi obtido com função rbf, padronização e discretização EW. Outro fato importante é o 
uso de 1500 neurônios com funções rbf na camada escondida, permitindo uma acurácia próxima a $94 \%$.

Tabela 40 - Resultados obtidos pelo classificador ELM com risco $C_{i j}=0.5+\frac{1}{\text { total }_{p}+\text { total }_{n}}$ para o conjunto de dados AID439 após a aplicação do rotulador PU.

\begin{tabular}{|c|c|c|c|c|c|c|c|c|c|c|c|c|c|c|}
\hline id & VP & FP & VN & FN & Pre. & Rec. & $t_{f p}$ & $t_{v p}$ & Acc & $f_{1}$ & Scale & Disc & Neu. & Fa. \\
\hline c0 & 11 & 2 & 54 & 2 & 0.85 & 0.85 & 0.04 & 0.85 & 0.94 & 0.85 & $\mathrm{~S}$ & $\mathrm{EW}$ & 1500 & $\mathrm{rbf}$ \\
c1 & 9 & 0 & 56 & 4 & 1.0 & 0.69 & 0.0 & 0.69 & 0.94 & 0.82 & $\mathrm{~S}$ & $\mathrm{EF}$ & 1000 & $\mathrm{rbf}$ \\
c2 & 11 & 3 & 53 & 2 & 0.79 & 0.85 & 0.05 & 0.85 & 0.93 & 0.81 & $\mathrm{~S}$ & $\mathrm{EW}$ & 1000 & $\mathrm{rbf}$ \\
c3 & 8 & 0 & 56 & 5 & 1.0 & 0.62 & 0.0 & 0.62 & 0.93 & 0.76 & $\mathrm{~S}$ & - & 1000 & $\mathrm{rbf}$ \\
c4 & 8 & 0 & 56 & 5 & 1.0 & 0.62 & 0.0 & 0.62 & 0.93 & 0.76 & $\mathrm{~S}$ & - & 1500 & $\mathrm{rbf}$ \\
c5 & 8 & 0 & 56 & 5 & 1.0 & 0.62 & 0.0 & 0.62 & 0.93 & 0.76 & $\mathrm{~S}$ & - & 500 & $\mathrm{rbf}$ \\
c6 & 8 & 0 & 56 & 5 & 1.0 & 0.62 & 0.0 & 0.62 & 0.93 & 0.76 & $\mathrm{~S}$ & - & 1000 & sig \\
c7 & 8 & 0 & 56 & 5 & 1.0 & 0.62 & 0.0 & 0.62 & 0.93 & 0.76 & $\mathrm{~S}$ & - & 1500 & sig \\
c8 & 8 & 0 & 56 & 5 & 1.0 & 0.62 & 0.0 & 0.62 & 0.93 & 0.76 & $\mathrm{~S}$ & - & 500 & sig \\
c9 & 11 & 5 & 51 & 2 & 0.69 & 0.85 & 0.09 & 0.85 & 0.9 & 0.76 & $\mathrm{~S}$ & $\mathrm{EW}$ & 500 & $\mathrm{rbf}$ \\
\hline
\end{tabular}

O desempenho dos melhores modelos também pode ser observado por meio da análise no espaço ROC, conforme a figura 20. Nota-se que, após a estimação dos rótulos via $\mathrm{PU}$, os pontos relativos aos classificadores avaliados moveram-se de áreas pouco favorecidas para áreas no espaço ROC associadas a melhores desempenhos. Mesmo que posicionados na área triangular acima do gráfico, os modelos selecionados utilizando a rotulação original dos dados adimitem até $50 \%$ dos elemetos positivos classificados incorretamente. Em alguns casos, observa-se que os classificadores se aproximam da linha diagonal pontilhada no gráfico, que indica uma abordagem aleatória para a classificação de novas instâncias. Por outro lado, observa-se também que utilizando a rotulação estimada os modelos selecionados reduzem sua taca de falsos positivos, melhorando os resultados de classificação empregando os rótulações anteriormente estimadas. 


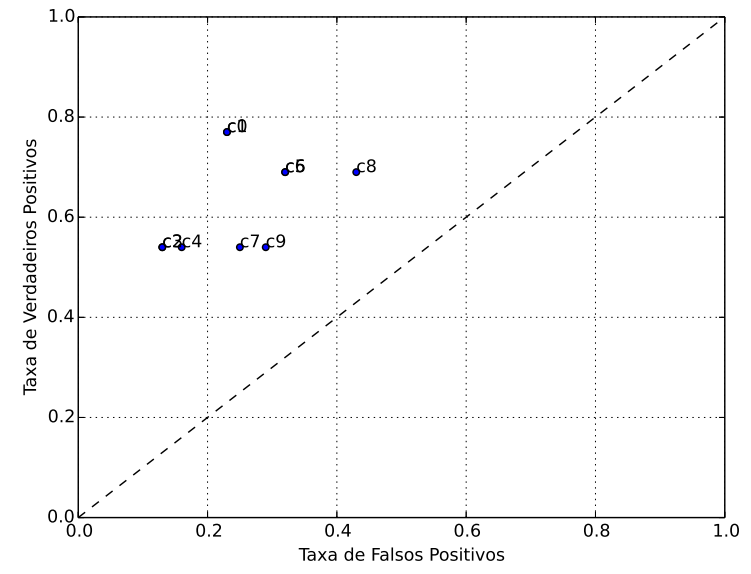

(a)

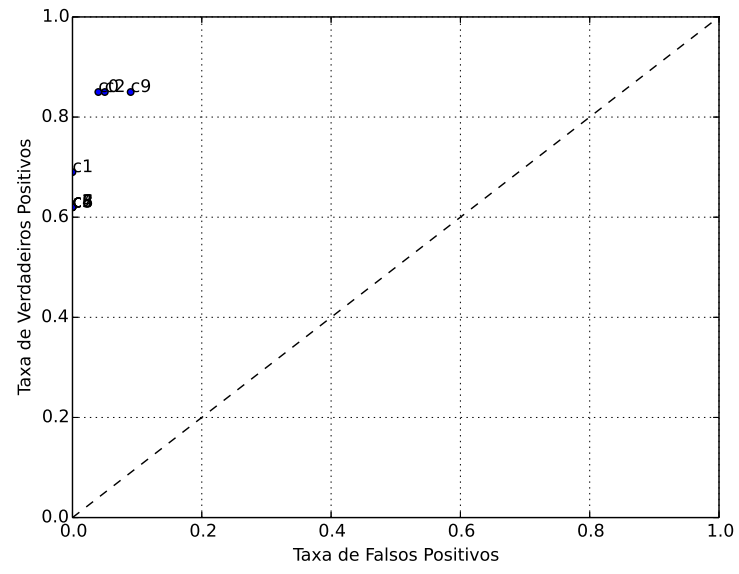

(b)

Figura 20 - (a)Modelos produzidos utilizando $C_{i j}=0.5+\frac{\text { total }_{p}}{\text { total }_{n}}$ para o conjunto de dados AID439. (b)Modelos utilizando $C_{i j}=0.5+\frac{\text { total }_{p}}{\text { total }_{n}}$ para o conjunto de dados AID439, com PU.

Cenário 3: Análise de classificadores ELM com fator de custo $C_{i j}=1.0$

Os resultados alcançados pelo classificador construído para os dados com rotulação original são apresentados na tabela 39. O melhor modelo apresentou acurácia igual a 88\%.

Tabela 41 - Resultados obtidos pelo classificador ELM com risco 1.0 para o conjunto de dados AID439 antes da aplicação do rotulador PU.

\begin{tabular}{|c|c|c|c|c|c|c|c|c|c|c|c|c|c|c|}
\hline id & VP & FP & VN & FN & Pre. & Rec. & $t_{f p}$ & $t_{v p}$ & Acc & $f_{1}$ & Scale & Disc & Neu. & Fa. \\
\hline c0 & 8 & 3 & 53 & 5 & 0.73 & 0.62 & 0.05 & 0.62 & 0.88 & 0.67 & $\mathrm{~S}$ & - & 1000 & rbf \\
c1 & 8 & 3 & 53 & 5 & 0.73 & 0.62 & 0.05 & 0.62 & 0.88 & 0.67 & $\mathrm{~S}$ & - & 500 & rbf \\
c2 & 8 & 3 & 53 & 5 & 0.73 & 0.62 & 0.05 & 0.62 & 0.88 & 0.67 & $\mathrm{~S}$ & - & 1000 & rbf \\
c3 & 8 & 3 & 53 & 5 & 0.73 & 0.62 & 0.05 & 0.62 & 0.88 & 0.67 & $\mathrm{~S}$ & - & 500 & rbf \\
c4 & 6 & 3 & 53 & 7 & 0.67 & 0.46 & 0.05 & 0.46 & 0.86 & 0.55 & $\mathrm{~S}$ & - & 1500 & rbf \\
c5 & 6 & 3 & 53 & 7 & 0.67 & 0.46 & 0.05 & 0.46 & 0.86 & 0.55 & $\mathrm{~S}$ & - & 1500 & rbf \\
c6 & 6 & 4 & 52 & 7 & 0.6 & 0.46 & 0.07 & 0.46 & 0.84 & 0.52 & $\mathrm{~S}$ & - & 500 & sig \\
c7 & 6 & 4 & 52 & 7 & 0.6 & 0.46 & 0.07 & 0.46 & 0.84 & 0.52 & $\mathrm{~S}$ & - & 500 & sig \\
c8 & 13 & 24 & 32 & 0 & 0.35 & 1.0 & 0.43 & 1.0 & 0.65 & 0.52 & - & EW & 1000 & sig \\
c9 & 13 & 24 & 32 & 0 & 0.35 & 1.0 & 0.43 & 1.0 & 0.65 & 0.52 & - & EW & 500 & sig \\
\hline
\end{tabular}

Para os experimentos após aplicação de PU, observa-se que o melhor experimento foi obtido com função rbf, padronização e discretização EF. Outro fato importante é o uso de 1500 neurônios com funções rbf na camada escondida, permitindo uma acurácia próxima a 100\%. Neste mesmo sentido, um outro classificador com 500 neurônios na camada escondida e funções sigmoidais produziu os mesmos resultados. 
Tabela 42 - Resultados obtidos pelo classificador ELM com risco 1.0 para o conjunto de dados AID439 após a aplicação do rotulador PU.

\begin{tabular}{|c|c|c|c|c|c|c|c|c|c|c|c|c|c|c|}
\hline id & VP & FP & VN & FN & Pre. & Rec. & $t_{f p}$ & $t_{v p}$ & Acc & $f_{1}$ & Scale & Disc & Neu. & Fa. \\
\hline c0 & 12 & 0 & 56 & 1 & 1.0 & 0.92 & 0.0 & 0.92 & 0.99 & 0.96 & $\mathrm{~S}$ & $\mathrm{EF}$ & 1500 & rbf \\
c1 & 12 & 0 & 56 & 1 & 1.0 & 0.92 & 0.0 & 0.92 & 0.99 & 0.96 & $\mathrm{~S}$ & $\mathrm{EF}$ & 500 & $\mathrm{sig}$ \\
c2 & 12 & 1 & 55 & 1 & 0.92 & 0.92 & 0.02 & 0.92 & 0.97 & 0.92 & - & $\mathrm{EW}$ & 500 & lin \\
c3 & 12 & 1 & 55 & 1 & 0.92 & 0.92 & 0.02 & 0.92 & 0.97 & 0.92 & $\mathrm{~S}$ & $\mathrm{EW}$ & 500 & $\mathrm{rbf}$ \\
c4 & 12 & 1 & 55 & 1 & 0.92 & 0.92 & 0.02 & 0.92 & 0.97 & 0.92 & $\mathrm{~S}$ & $\mathrm{EW}$ & 1500 & $\mathrm{sig}$ \\
c5 & 12 & 1 & 55 & 1 & 0.92 & 0.92 & 0.02 & 0.92 & 0.97 & 0.92 & $\mathrm{~S}$ & $\mathrm{EW}$ & 500 & $\mathrm{sig}$ \\
c6 & 11 & 0 & 56 & 2 & 1.0 & 0.85 & 0.0 & 0.85 & 0.97 & 0.92 & $\mathrm{~S}$ & $\mathrm{EF}$ & 1000 & sig \\
c7 & 12 & 1 & 55 & 1 & 0.92 & 0.92 & 0.02 & 0.92 & 0.97 & 0.92 & - & $\mathrm{EW}$ & 500 & lin \\
c8 & 11 & 1 & 55 & 2 & 0.92 & 0.85 & 0.02 & 0.85 & 0.96 & 0.88 & $\mathrm{~S}$ & $\mathrm{EW}$ & 1000 & sig \\
c9 & 10 & 0 & 56 & 3 & 1.0 & 0.77 & 0.0 & 0.77 & 0.96 & 0.87 & $\mathrm{~S}$ & $\mathrm{EW}$ & 1000 & $\mathrm{rbf}$ \\
\hline
\end{tabular}

O desempenho dos melhores modelos também pode ser observado por meio da análise no espaço ROC, conforme a figura 21. Nota-se que, após a estimação dos rótulos via PU, os pontos relativos aos classificadores avaliados moveram-se de áreas pouco favorecidas para áreas no espaço ROC associadas a melhores desempenhos. Sem a etapa de estimação dos rótulos, apesar dos modelos identificarem todos os elementos positivos corretamente, aproximadamente $40 \%$ das instâncias negativas foram identificadas incorretamente. Considerando a correção na rotulação, observa-se que os melhores modelos obtveram resultados expressivos, apresentando um número de falsos positivos consideravelmente menor após a aplicação do estimador PU.

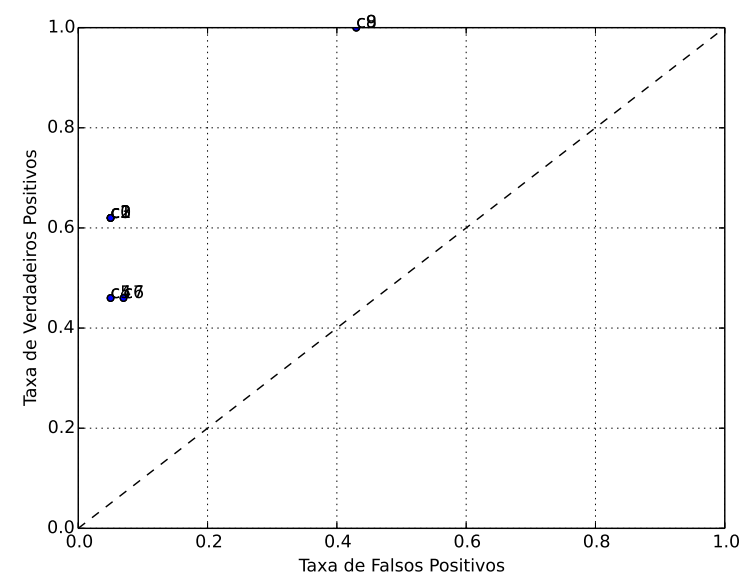

(a)

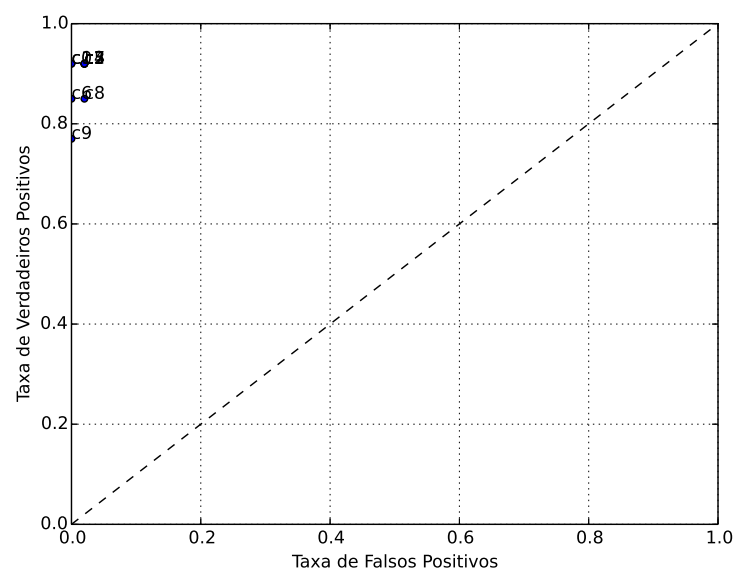

(b)

Figura 21 - (a)Modelos produzidos utilizando $C_{i j}=1.0$ para o conjunto de dados AID439. (b)Modelos utilizando $C_{i j}=1.0$ para o conjunto de dados AID439, com PU. 
Cenário 3: Análise de classificadores ELM com fator de custo $C_{i j}=1.0+\frac{1}{\text { total }_{p}+\text { total }_{n}}$

Os resultados alcançados pelo classificador construído para os dados com rotulação original são apresentados na tabela 39. O melhor modelo apresentou acurácia igual a 90\%.

Tabela 43 - Resultados obtidos pelo classificador ELM com risco $C_{i j}=1.0+\frac{1}{\text { total }_{p}+\text { total }_{n}}$ para o conjunto de dados AID439 antes da aplicação do rotulador PU.

\begin{tabular}{|c|c|c|c|c|c|c|c|c|c|c|c|c|c|c|}
\hline id & VP & FP & VN & FN & Pre. & Rec. & $t_{f p}$ & $t_{v p}$ & Acc & $f_{1}$ & Scale & Disc & Neu. & Fa. \\
\hline c0 & 7 & 1 & 55 & 6 & 0.88 & 0.54 & 0.02 & 0.54 & 0.9 & 0.67 & $\mathrm{~S}$ & - & 1000 & $\mathrm{sig}$ \\
$\mathrm{c} 1$ & 7 & 1 & 55 & 6 & 0.88 & 0.54 & 0.02 & 0.54 & 0.9 & 0.67 & $\mathrm{~S}$ & - & 1000 & $\mathrm{sig}$ \\
$\mathrm{c} 2$ & 6 & 0 & 56 & 7 & 1.0 & 0.46 & 0.0 & 0.46 & 0.9 & 0.63 & $\mathrm{~S}$ & - & 1000 & $\mathrm{rbf}$ \\
$\mathrm{c} 3$ & 6 & 0 & 56 & 7 & 1.0 & 0.46 & 0.0 & 0.46 & 0.9 & 0.63 & $\mathrm{~S}$ & - & 1500 & $\mathrm{rbf}$ \\
$\mathrm{c} 4$ & 6 & 0 & 56 & 7 & 1.0 & 0.46 & 0.0 & 0.46 & 0.9 & 0.63 & $\mathrm{~S}$ & - & 500 & $\mathrm{rbf}$ \\
$\mathrm{c} 5$ & 6 & 0 & 56 & 7 & 1.0 & 0.46 & 0.0 & 0.46 & 0.9 & 0.63 & $\mathrm{~S}$ & - & 1500 & $\mathrm{sig}$ \\
$\mathrm{c} 6$ & 6 & 0 & 56 & 7 & 1.0 & 0.46 & 0.0 & 0.46 & 0.9 & 0.63 & $\mathrm{~S}$ & - & 500 & $\mathrm{sig}$ \\
$\mathrm{c} 7$ & 6 & 0 & 56 & 7 & 1.0 & 0.46 & 0.0 & 0.46 & 0.9 & 0.63 & $\mathrm{~S}$ & - & 1000 & $\mathrm{rbf}$ \\
$\mathrm{c} 8$ & 6 & 0 & 56 & 7 & 1.0 & 0.46 & 0.0 & 0.46 & 0.9 & 0.63 & $\mathrm{~S}$ & - & 1500 & $\mathrm{rbf}$ \\
$\mathrm{c} 9$ & 6 & 0 & 56 & 7 & 1.0 & 0.46 & 0.0 & 0.46 & 0.9 & 0.63 & $\mathrm{~S}$ & - & 500 & $\mathrm{rbf}$ \\
\hline
\end{tabular}

Para os experimentos após aplicação de PU, observa-se que o melhor experimento foi obtido com função sigmoidal e discretização EF, Sem necessidade de padronização, este modelo obteve $97 \%$ de acurácia sem apresentar nenhum elemento classificado como falso positivo.

Tabela 44 - Resultados obtidos pelo classificador ELM com risco $C_{i j}=1.0+\frac{1}{\text { total }_{p}+\text { total }_{n}}$ para o conjunto de dados AID439 após a aplicação do rotulador PU.

\begin{tabular}{|c|c|c|c|c|c|c|c|c|c|c|c|c|c|c|}
\hline id & VP & FP & VN & FN & Pre. & Rec. & $t_{f p}$ & $t_{v p}$ & Acc & $f_{1}$ & Scale & Disc & Neu. & Fa. \\
\hline c0 & 11 & 0 & 56 & 2 & 1.0 & 0.85 & 0.0 & 0.85 & 0.97 & 0.92 & - & EF & 1500 & sig \\
c1 & 11 & 0 & 56 & 2 & 1.0 & 0.85 & 0.0 & 0.85 & 0.97 & 0.92 & - & EF & 1500 & sig \\
c2 & 12 & 1 & 55 & 1 & 0.92 & 0.92 & 0.02 & 0.92 & 0.97 & 0.92 & S & EW & 1000 & sig \\
c3 & 11 & 1 & 55 & 2 & 0.92 & 0.85 & 0.02 & 0.85 & 0.96 & 0.88 & S & EF & 1000 & sig \\
c4 & 9 & 0 & 56 & 4 & 1.0 & 0.69 & 0.0 & 0.69 & 0.94 & 0.82 & S & EF & 1500 & rbf \\
c5 & 9 & 0 & 56 & 4 & 1.0 & 0.69 & 0.0 & 0.69 & 0.94 & 0.82 & S & EF & 500 & rbf \\
c6 & 9 & 0 & 56 & 4 & 1.0 & 0.69 & 0.0 & 0.69 & 0.94 & 0.82 & - & EF & 1500 & rbf \\
c7 & 9 & 0 & 56 & 4 & 1.0 & 0.69 & 0.0 & 0.69 & 0.94 & 0.82 & - & EF & 500 & rbf \\
c8 & 9 & 0 & 56 & 4 & 1.0 & 0.69 & 0.0 & 0.69 & 0.94 & 0.82 & - & EF & 1000 & sig \\
c9 & 9 & 0 & 56 & 4 & 1.0 & 0.69 & 0.0 & 0.69 & 0.94 & 0.82 & - & EF & 1500 & rbf \\
\hline
\end{tabular}

O desempenho dos melhores modelos também pode ser observado por meio da análise no espaço ROC, conforme a figura 22. Nota-se que, após a estimação dos rótulos via PU, os pontos relativos aos classificadores avaliados moveram-se de áreas pouco favorecidas para áreas no espaço ROC associadas a melhores desempenhos. Avaliando o 
desempenho dos classificadores produzidos sob o espaço ROC, é possível observar que o melhor classificador, aqui identificado com c0, é posicionado em uma área do gráfico dita conservadora. Mais especificamente, o melhor classificador necessita de mais evidências para classificar uma instância como positiva, produzindo assim uma taxa de falso positivo baixa.

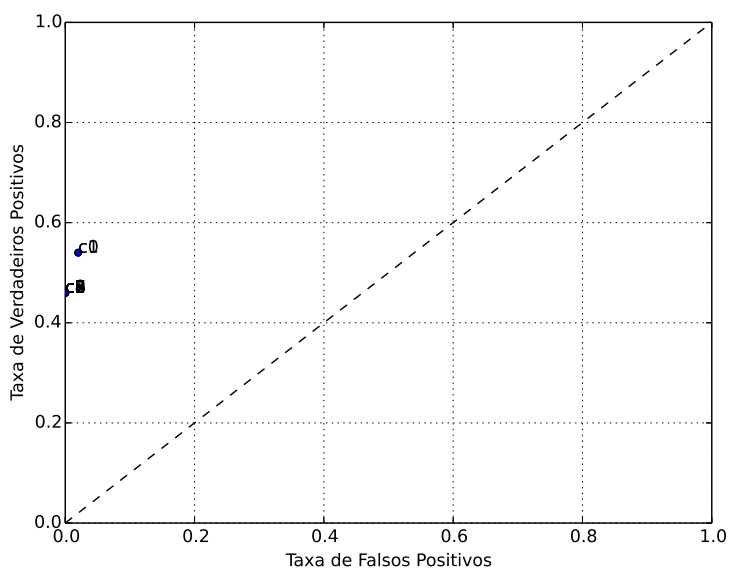

(a)

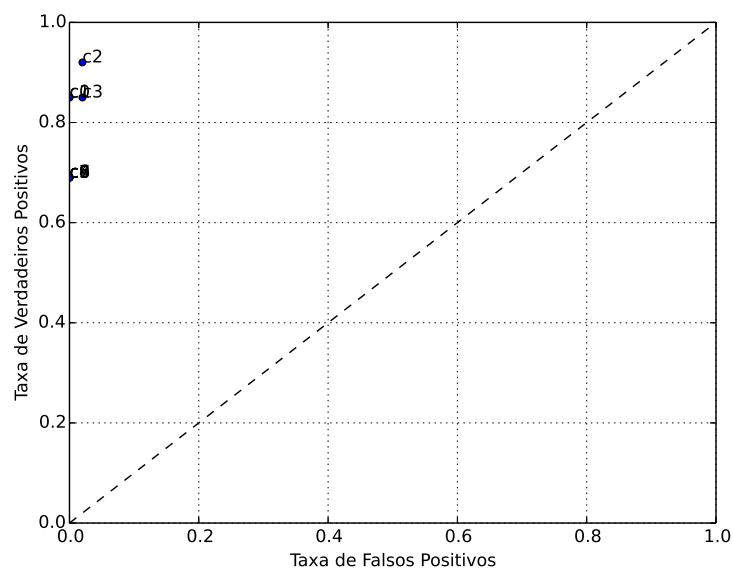

(b)

Figura 22 - (a)Modelos produzidos utilizando $C_{i j}=1.0+\frac{t_{\text {total }}}{\text { total }_{n}}$ para o conjunto de dados AID439. (b)Modelos utilizando $C_{i j}=1.0+\frac{\text { total }_{p}}{\text { total }_{n}}$ para o conjunto de dados AID439, com PU.

Em uma análise geral para o conjunto de dados AID439, foi possível observar que o custo $C_{i j}=1.0$ foi suficiente para levar o classificador próximo à $99 \%$ de acerto. Assim como em exemplos com alta taxa de acertos citados anteriormente, os melhores resultados ficaram por conta da configuração de dados que contemplou padronização e discretização EF. Além dos resultados superarem o erro majoritário de 81,15\%, outro destaque ficou para o uso da função rbf e utilização de 1500 neurônios na camada escondida.

\subsubsection{Experimentos de rotulação via PU - Conjunto de dados AID1608}

A tabela 45 apresenta as distribuições obtidas após o processo PU, considerando a rotulação original do conjunto, que possui 68 elementos positivos e 965 elementos negativos: 
Tabela 45 - Distribuições de classes obtidas após a execução do processo PU contemplando a variação dos custos para o conjunto de dados AID1608.

\begin{tabular}{|c|c|c|c|c|}
\hline $\begin{array}{ll}\text { Conjunto de dados } & \text { Custo } \\
\end{array}$ & $\frac{1}{\text { total }_{p}+\text { total }_{n}}$ & $0.5+\frac{\text { total }_{p}}{\text { total }_{n}}$ & 1.0 & $1.0+\frac{\text { total }_{p}}{\text { total }_{n}}$ \\
\hline Bin. e Cont. + EW & $994 / 39$ & $159 / 874$ & $80 / 953$ & $78 / 955$ \\
\hline Cont. + Scale & $68 / 965$ & $85 / 948$ & $84 / 949$ & $173 / 860$ \\
\hline Bin. & $1023 / 10$ & $94 / 939$ & $94 / 939$ & $91 / 942$ \\
\hline Bin. e Cont. & $1012 / 21$ & $96 / 937$ & $94 / 939$ & $81 / 952$ \\
\hline Cont. & $68 / 965$ & $77 / 956$ & $75 / 958$ & $85 / 948$ \\
\hline Bin. e Cont. + Scale & $57 / 305$ & $60 / 302$ & $60 / 302$ & $62 / 300$ \\
\hline Cont. + Scale & $57 / 305$ & $102 / 260$ & $58 / 304$ & $58 / 304$ \\
\hline Bin. e Con. + EF & $1017 / 16$ & $80 / 953$ & $82 / 951$ & $101 / 932$ \\
\hline Bin. e Cont. + Scale + EF & $1001 / 32$ & $83 / 950$ & $130 / 903$ & $108 / 925$ \\
\hline Bin. e Cont. + Scale + EW & $1025 / 8$ & $182 / 851$ & $180 / 853$ & $192 / 841$ \\
\hline Cont. $+\mathrm{EF}$ & $186 / 20$ & $174 / 859$ & $89 / 944$ & $107 / 926$ \\
\hline Cont. + Scale + EW & $1009 / 24$ & $76 / 957$ & $276 / 757$ & $73 / 960$ \\
\hline Cont. + Scale + EF & $493 / 540$ & $71 / 962$ & $69 / 964$ & $75 / 958$ \\
\hline Cont. + EW & $1008 / 25$ & $81 / 952$ & $81 / 952$ & $83 / 950$ \\
\hline
\end{tabular}

Nesses experimentos, durante a etapa de estimação de rótulos, foi possível observar que para o custo $\frac{1}{\text { total }_{p}+\text { total }_{n}}$ as rotulações se alteram bruscamente invertendo a distribuição das classes. Mesmo em cenários nos quais as alterações de rótulos são menores, ainda assim a distribuição após o experimento se distancia da distribuição original.

\subsubsection{Experimentos de classificação via ELM - Conjunto de dados AID1608}

Nos próximos experimentos, classificadores ELM serão construídos para o conjunto de dados AID1608, levando em conta sua rotulação original e as rotulações estimadas pelo método PU. Objetiva-se, com essa análise, avaliar o quanto esses diferentes cenários afetam o desempenho final do modelo de classificação.

Cenário 1: Análise de classificadores ELM com fator de custo $C_{i j}=\frac{1}{\text { total }_{p}+\text { total }_{n}}$

Os resultados alcançados pelo classificador construído para os dados com rotulação original são apresentados na tabela 37. O melhor modelo apresentou acurácia igual a 95\%. 
Tabela 46 - Resultados obtidos pelo classificador ELM com risco $C_{i j}=\frac{1}{\text { total }_{p}+\text { total }_{n}}$ para o conjunto de dados AID1608 antes da aplicação do rotulador PU.

\begin{tabular}{|c|c|c|c|c|c|c|c|c|c|c|c|c|c|c|}
\hline id & VP & FP & VN & FN & Pre. & Rec. & $t_{f p}$ & $t_{v p}$ & Acc & $f_{1}$ & Scale & Disc & Neu. & Fa. \\
\hline c0 & 16 & 1 & 964 & 52 & 0.94 & 0.24 & 0.0 & 0.24 & 0.95 & 0.38 & $\mathrm{~S}$ & - & 1000 & sig \\
c1 & 16 & 1 & 964 & 52 & 0.94 & 0.24 & 0.0 & 0.24 & 0.95 & 0.38 & $\mathrm{~S}$ & - & 1000 & sig \\
c2 & 15 & 2 & 963 & 53 & 0.88 & 0.22 & 0.0 & 0.22 & 0.95 & 0.35 & $\mathrm{~S}$ & - & 1500 & sig \\
c3 & 15 & 2 & 963 & 53 & 0.88 & 0.22 & 0.0 & 0.22 & 0.95 & 0.35 & $\mathrm{~S}$ & - & 1500 & sig \\
c4 & 29 & 108 & 857 & 39 & 0.21 & 0.43 & 0.11 & 0.43 & 0.86 & 0.28 & $\mathrm{~S}$ & - & 1000 & sig \\
c5 & 29 & 108 & 857 & 39 & 0.21 & 0.43 & 0.11 & 0.43 & 0.86 & 0.28 & $\mathrm{~S}$ & - & 1000 & sig \\
c6 & 10 & 0 & 965 & 58 & 1.0 & 0.15 & 0.0 & 0.15 & 0.94 & 0.26 & - & - & 1500 & sig \\
c7 & 24 & 96 & 869 & 44 & 0.2 & 0.35 & 0.1 & 0.35 & 0.86 & 0.26 & $\mathrm{~S}$ & - & 1500 & sig \\
c8 & 24 & 96 & 869 & 44 & 0.2 & 0.35 & 0.1 & 0.35 & 0.86 & 0.26 & $\mathrm{~S}$ & - & 1500 & sig \\
c9 & 16 & 37 & 928 & 52 & 0.3 & 0.24 & 0.04 & 0.24 & 0.91 & 0.26 & $\mathrm{~S}$ & EW & 1000 & sig \\
\hline
\end{tabular}

Para os experimentos após aplicação de PU, apesar do número de falso positivos e falsos negativos, o modelo atingiu 99\% de acurácia. O melhor classificador utilizou discretização EF, padronização e função sigmoidal nos 1500 neurônios da camada escondida.

Tabela 47 - Resultados obtidos pelo classificador ELM com risco $C_{i j}=\frac{1}{\text { total }_{p}+\text { total }_{n}}$ para o conjunto de dados AID1608 após a aplicação do rotulador PU.

\begin{tabular}{|c|c|c|c|c|c|c|c|c|c|c|c|c|c|c|}
\hline id & VP & FP & VN & FN & Pre. & Rec. & $t_{f p}$ & $t_{v p}$ & Acc & $f_{1}$ & Scale & Disc & Neu. & Fa. \\
\hline c0 & 65 & 6 & 959 & 3 & 0.92 & 0.96 & 0.01 & 0.96 & 0.99 & 0.94 & $\mathrm{~S}$ & $\mathrm{EF}$ & 1500 & sig \\
c1 & 64 & 6 & 959 & 4 & 0.91 & 0.94 & 0.01 & 0.94 & 0.99 & 0.93 & $\mathrm{~S}$ & $\mathrm{EF}$ & 1000 & $\mathrm{sig}$ \\
c2 & 65 & 7 & 958 & 3 & 0.9 & 0.96 & 0.01 & 0.96 & 0.99 & 0.93 & - & $\mathrm{EW}$ & 1000 & sig \\
c3 & 65 & 7 & 958 & 3 & 0.9 & 0.96 & 0.01 & 0.96 & 0.99 & 0.93 & - & $\mathrm{EW}$ & 1000 & sig \\
c4 & 66 & 13 & 952 & 2 & 0.84 & 0.97 & 0.01 & 0.97 & 0.99 & 0.9 & - & $\mathrm{EW}$ & 1500 & sig \\
c5 & 66 & 13 & 952 & 2 & 0.84 & 0.97 & 0.01 & 0.97 & 0.99 & 0.9 & - & $\mathrm{EW}$ & 1500 & sig \\
c6 & 51 & 3 & 962 & 17 & 0.94 & 0.75 & 0.0 & 0.75 & 0.98 & 0.84 & - & $\mathrm{EF}$ & 1500 & sig \\
c7 & 51 & 3 & 962 & 17 & 0.94 & 0.75 & 0.0 & 0.75 & 0.98 & 0.84 & - & $\mathrm{EF}$ & 1500 & sig \\
c8 & 51 & 8 & 957 & 17 & 0.86 & 0.75 & 0.01 & 0.75 & 0.98 & 0.8 & - & $\mathrm{EF}$ & 1000 & sig \\
c9 & 51 & 8 & 957 & 17 & 0.86 & 0.75 & 0.01 & 0.75 & 0.98 & 0.8 & - & $\mathrm{EF}$ & 1000 & sig \\
\hline
\end{tabular}

O desempenho dos melhores modelos também pode ser observado por meio da análise no espaço ROC, conforme a figura 23. Nota-se que, após a estimação dos rótulos via PU, os pontos relativos aos classificadores avaliados moveram-se de áreas pouco favorecidas para áreas no espaço ROC associadas a melhores desempenhos. É possível perceber, que de forma geral, o processo de estimação de rótulos melhora o resultado final dos classificadores. Neste experimento, apesar de nem todos os classificadores atingirem taxa de verdadeiro positivo acima de $80 \%$, todos apresentam baixas taxa de falsos positivos. 


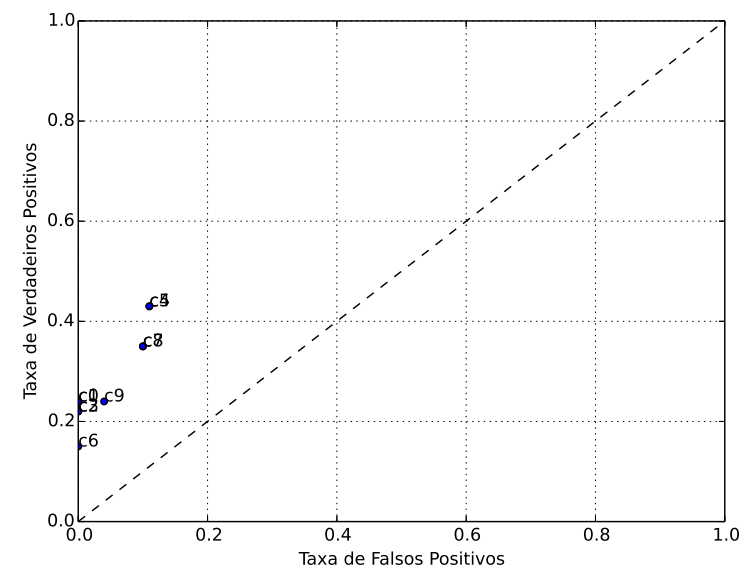

(a)

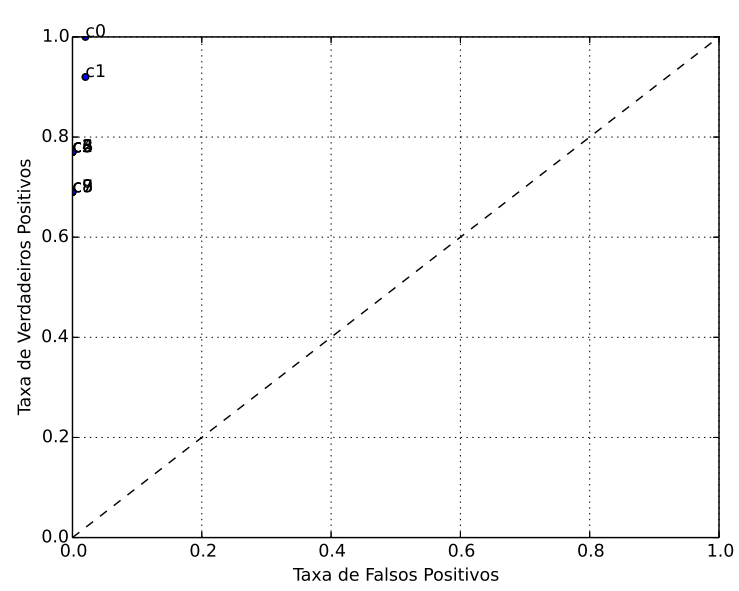

(b)

Figura 23 - (a)Modelos utilizando $C_{i j}=\frac{1}{\text { total }_{p}+\text { total }_{n}}$ para o conjunto de dados AID1608.(b)Modelos utilizando $C_{i j}=\frac{1}{\text { total }_{p}+\text { total }_{n}}$ para o conjunto de dados AID1608, com PU.

Cenário 2: Análise de classificadores ELM com fator de custo $C_{i j}=0.5+\frac{1}{\text { total }_{p}+\text { total }_{n}}$

Os resultados alcançados pelo classificador construído para os dados com rotulação original são apresentados na tabela 37. O melhor modelo apresentou acurácia igual a 96\%.

Tabela 48 - Resultados obtidos pelo classificador ELM com risco $C_{i j}=0.5+\frac{1}{\text { total }_{p}+\text { total }_{n}}$ para o conjunto de dados AID1608 antes da aplicação do rotulador PU.

\begin{tabular}{|c|c|c|c|c|c|c|c|c|c|c|c|c|c|c|}
\hline id & VP & FP & VN & FN & Pre. & Rec. & $t_{f p}$ & $t_{v p}$ & Acc & $f_{1}$ & Scale & Disc & Neu. & Fa. \\
\hline c0 & 44 & 14 & 951 & 24 & 0.76 & 0.65 & 0.01 & 0.65 & 0.96 & 0.7 & $\mathrm{~S}$ & - & 1500 & sig \\
c1 & 44 & 14 & 951 & 24 & 0.76 & 0.65 & 0.01 & 0.65 & 0.96 & 0.7 & $\mathrm{~S}$ & - & 1500 & $\mathrm{sig}$ \\
c2 & 56 & 0 & 1930 & 80 & 1.0 & 0.41 & 0.0 & 0.41 & 0.96 & 0.58 & - & - & 1000 & rbf \\
c3 & 56 & 0 & 1930 & 80 & 1.0 & 0.41 & 0.0 & 0.41 & 0.96 & 0.58 & - & - & 1500 & rbf \\
c4 & 56 & 0 & 1930 & 80 & 1.0 & 0.41 & 0.0 & 0.41 & 0.96 & 0.58 & - & - & 500 & rbf \\
c5 & 56 & 0 & 1930 & 80 & 1.0 & 0.41 & 0.0 & 0.41 & 0.96 & 0.58 & - & - & 1000 & sig \\
c6 & 56 & 0 & 1930 & 80 & 1.0 & 0.41 & 0.0 & 0.41 & 0.96 & 0.58 & - & - & 500 & sig \\
c7 & 57 & 6 & 1924 & 79 & 0.9 & 0.42 & 0.0 & 0.42 & 0.96 & 0.57 & - & - & 1500 & sig \\
c8 & 28 & 4 & 961 & 40 & 0.88 & 0.41 & 0.0 & 0.41 & 0.96 & 0.56 & $\mathrm{~S}$ & - & 1000 & sig \\
c9 & 28 & 4 & 961 & 40 & 0.88 & 0.41 & 0.0 & 0.41 & 0.96 & 0.56 & $\mathrm{~S}$ & - & 1000 & sig \\
\hline
\end{tabular}

Para os experimentos após aplicação de PU, nenhum dos modelos selecionados possuiam padronização em sua faze de pré-processamento. A discretização utilizada foi EW e o melhor desempenho foi obtido utilizando apenas 500 neurônios de função sigmoidal na camada escondida. 
Tabela 49 - Resultados obtidos pelo classificador ELM com risco $C_{i j}=0.5+\frac{1}{\text { total }_{p}+\text { total }_{n}}$ para o conjunto de dados AID1608 após a aplicação do rotulador PU.

\begin{tabular}{|c|c|c|c|c|c|c|c|c|c|c|c|c|c|c|}
\hline id & VP & FP & VN & FN & Pre. & Rec. & $t_{f p}$ & $t_{v p}$ & Acc & $f_{1}$ & Scale & Disc & Neu. & Fa. \\
\hline c0 & 66 & 0 & 965 & 2 & 1.0 & 0.97 & 0.0 & 0.97 & 1.0 & 0.99 & - & EF & 500 & sig \\
c1 & 66 & 0 & 965 & 2 & 1.0 & 0.97 & 0.0 & 0.97 & 1.0 & 0.99 & - & EF & 500 & sig \\
c2 & 68 & 1 & 964 & 0 & 0.99 & 1.0 & 0.0 & 1.0 & 1.0 & 0.99 & - & EW & 1000 & sig \\
c3 & 67 & 0 & 965 & 1 & 1.0 & 0.99 & 0.0 & 0.99 & 1.0 & 0.99 & - & EW & 1500 & sig \\
c4 & 68 & 1 & 964 & 0 & 0.99 & 1.0 & 0.0 & 1.0 & 1.0 & 0.99 & - & EW & 1000 & sig \\
c5 & 67 & 0 & 965 & 1 & 1.0 & 0.99 & 0.0 & 0.99 & 1.0 & 0.99 & - & EW & 1500 & sig \\
c6 & 66 & 1 & 964 & 2 & 0.99 & 0.97 & 0.0 & 0.97 & 1.0 & 0.98 & - & EF & 1000 & sig \\
c7 & 66 & 1 & 964 & 2 & 0.99 & 0.97 & 0.0 & 0.97 & 1.0 & 0.98 & - & EF & 1500 & sig \\
c8 & 66 & 1 & 964 & 2 & 0.99 & 0.97 & 0.0 & 0.97 & 1.0 & 0.98 & - & EF & 1000 & sig \\
c9 & 66 & 1 & 964 & 2 & 0.99 & 0.97 & 0.0 & 0.97 & 1.0 & 0.98 & - & EF & 1500 & sig \\
\hline
\end{tabular}

O desempenho dos melhores modelos também pode ser observado por meio da análise no espaço ROC, conforme a figura 25. Nota-se que, após a estimação dos rótulos via PU, os pontos relativos aos classificadores avaliados moveram-se de áreas pouco favorecidas para áreas no espaço ROC associadas a melhores desempenhos. Observa-se que antes da aplicação do processo PU, apesar da baixa tava de falsos positivos, os classificadores selecionados identificaram menos de $70 \%$ dos elementos positivos existentes no conjunto de dados. Entretanto, após a estimação dos rótulos por meio do processo PU, a taxa de verdadeiros positivos atingiu níveis próximos a $100 \%$.

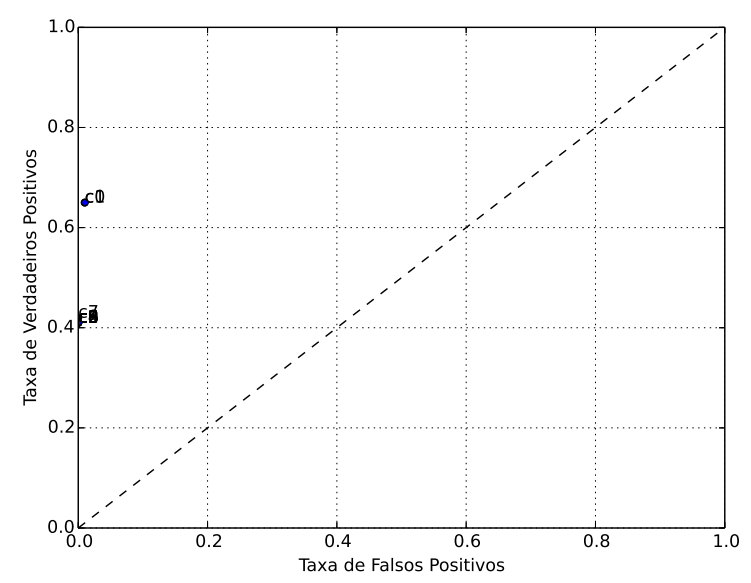

(a)

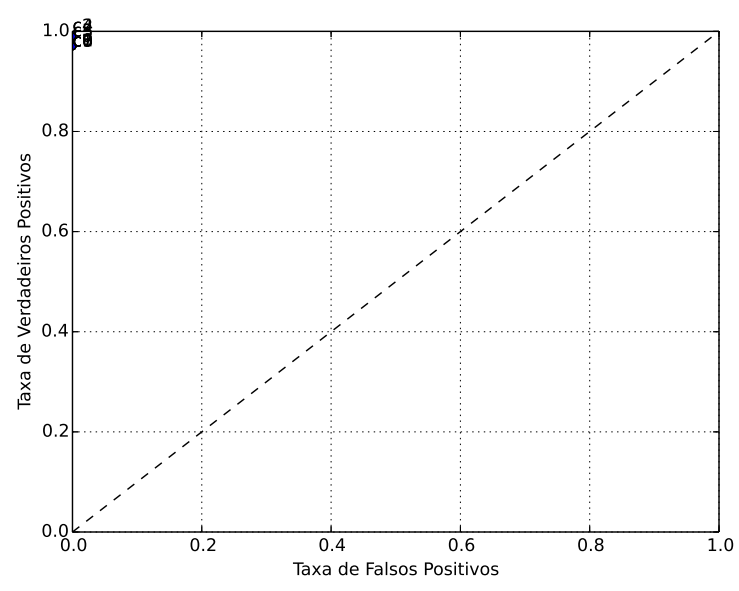

(b)

Figura 24 - (a)Modelos utilizando $C_{i j}=0.5+\frac{t_{\text {total }}}{\text { total }_{n}}$ para o conjunto de dados AID1608. (b)Modelos utilizando $C_{i j}=0.5+\frac{t_{\text {total }}}{\text { total }_{n}}$ para o conjunto de dados AID1608, com PU. 
Cenário 3: Análise de classificadores ELM com fator de custo $C_{i j}=1$

Os resultados alcançados pelo classificador construído para os dados com rotulação original são apresentados na tabela 37. O melhor modelo apresentou acurácia igual a 96\%.

Tabela 50 - Resultados obtidos pelo classificador ELM com risco 1.0 para o conjunto de dados AID1608 antes da aplicação do rotulador PU.

\begin{tabular}{|c|c|c|c|c|c|c|c|c|c|c|c|c|c|c|}
\hline id & VP & FP & VN & FN & Pre. & Rec. & $t_{f p}$ & $t_{v p}$ & Acc & $f_{1}$ & Scale & Disc & Neu. & Fa. \\
\hline c0 & 23 & 6 & 638 & 22 & 0.79 & 0.51 & 0.01 & 0.51 & 0.96 & 0.62 & $\mathrm{~S}$ & EF & 1500 & sig \\
c1 & 14 & 12 & 632 & 31 & 0.54 & 0.31 & 0.02 & 0.31 & 0.94 & 0.39 & S & EF & 500 & sig \\
c2 & 31 & 79 & 886 & 37 & 0.28 & 0.46 & 0.08 & 0.46 & 0.89 & 0.35 & S & EW & 1000 & sig \\
c3 & 13 & 17 & 627 & 32 & 0.43 & 0.29 & 0.03 & 0.29 & 0.93 & 0.35 & S & EF & 1000 & sig \\
c4 & 12 & 42 & 923 & 56 & 0.22 & 0.18 & 0.04 & 0.18 & 0.91 & 0.2 & S & - & 500 & sig \\
c5 & 12 & 42 & 923 & 56 & 0.22 & 0.18 & 0.04 & 0.18 & 0.91 & 0.2 & S & - & 500 & sig \\
c6 & 7 & 2 & 963 & 61 & 0.78 & 0.1 & 0.0 & 0.1 & 0.94 & 0.18 & - & - & 1000 & sig \\
c7 & 38 & 323 & 642 & 30 & 0.11 & 0.56 & 0.33 & 0.56 & 0.66 & 0.18 & - & EF & 500 & lin \\
c8 & 38 & 323 & 642 & 30 & 0.11 & 0.56 & 0.33 & 0.56 & 0.66 & 0.18 & - & EF & 500 & lin \\
c9 & 19 & 132 & 833 & 49 & 0.13 & 0.28 & 0.14 & 0.28 & 0.82 & 0.17 & S & EF & 1500 & lin \\
\hline
\end{tabular}

Para os experimentos após aplicação de PU, apesar da acurácia do modelo com melhor desempenho estar próxima de 100\%, a quantidade de neurônios na camada escondida foi de 1500, um número maior que outros classificadores semelhantes utilizando outros custos. Esse modelo também utilizou em sua fase de pré-processamento padronização e discretização EF.

Tabela 51 - Resultados obtidos pelo classificador ELM com risco 1.0 para o conjunto de dados AID1608 após a aplicação do rotulador PU.

\begin{tabular}{|c|c|c|c|c|c|c|c|c|c|c|c|c|c|c|}
\hline id & VP & FP & VN & FN & Pre. & Rec. & $t_{f p}$ & $t_{v p}$ & Acc & $f_{1}$ & Scale & Disc & Neu. & Fa. \\
\hline c0 & 66 & 1 & 964 & 2 & 0.99 & 0.97 & 0.0 & 0.97 & 1.0 & 0.98 & $\mathrm{~S}$ & EF & 1500 & sig \\
c1 & 65 & 0 & 965 & 3 & 1.0 & 0.96 & 0.0 & 0.96 & 1.0 & 0.98 & - & EW & 1500 & sig \\
c2 & 65 & 0 & 965 & 3 & 1.0 & 0.96 & 0.0 & 0.96 & 1.0 & 0.98 & - & EW & 1500 & sig \\
c3 & 66 & 2 & 963 & 2 & 0.97 & 0.97 & 0.0 & 0.97 & 1.0 & 0.97 & S & EF & 1000 & rbf \\
c4 & 65 & 1 & 964 & 3 & 0.98 & 0.96 & 0.0 & 0.96 & 1.0 & 0.97 & S & EF & 500 & sig \\
c5 & 65 & 3 & 962 & 3 & 0.96 & 0.96 & 0.0 & 0.96 & 0.99 & 0.96 & $\mathrm{~S}$ & EF & 1500 & rbf \\
c6 & 64 & 1 & 964 & 4 & 0.98 & 0.94 & 0.0 & 0.94 & 1.0 & 0.96 & $\mathrm{~S}$ & $\mathrm{EF}$ & 1000 & sig \\
c7 & 65 & 6 & 959 & 3 & 0.92 & 0.96 & 0.01 & 0.96 & 0.99 & 0.94 & - & $\mathrm{EF}$ & 1500 & sig \\
c8 & 65 & 6 & 959 & 3 & 0.92 & 0.96 & 0.01 & 0.96 & 0.99 & 0.94 & - & $\mathrm{EF}$ & 1500 & sig \\
c9 & 65 & 7 & 958 & 3 & 0.9 & 0.96 & 0.01 & 0.96 & 0.99 & 0.93 & - & $\mathrm{EF}$ & 1000 & sig \\
\hline
\end{tabular}

O desempenho dos melhores modelos também pode ser observado por meio da análise no espaço ROC, conforme a figura 25. Nota-se que, após a estimação dos rótulos via PU, os pontos relativos aos classificadores avaliados moveram-se de áreas pouco favorecidas 
para áreas no espaço ROC associadas a melhores desempenhos. Nesse experimeno, observase que boa parte dos modelos selecionados, independente das técnicas utilizadas em sua fase de pré-processamento, são posicionados na região que indica uma alta taxa de verdadeiros positivos e uma quantidade menor de falsos positivos. Este posicionamento indica que para os modelos selecionados, necessita-se de fortes evidências para classificar as instâncias desse conjunto de dados como ativas ou inativas.

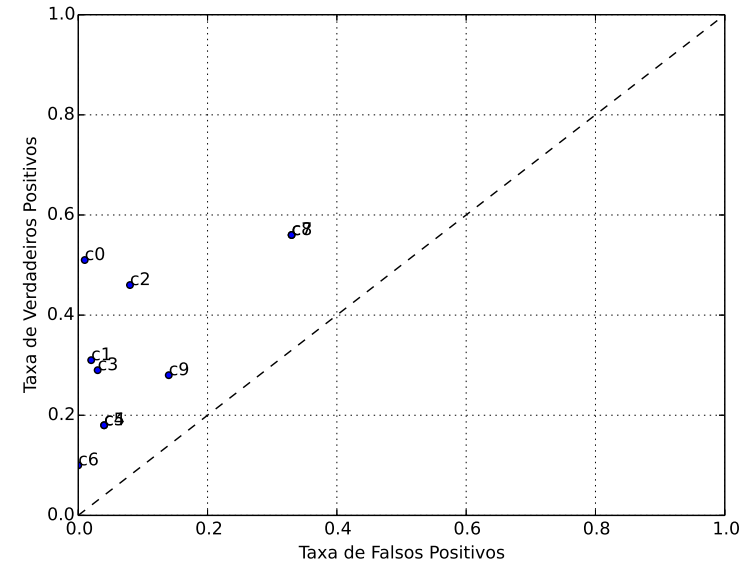

(a)

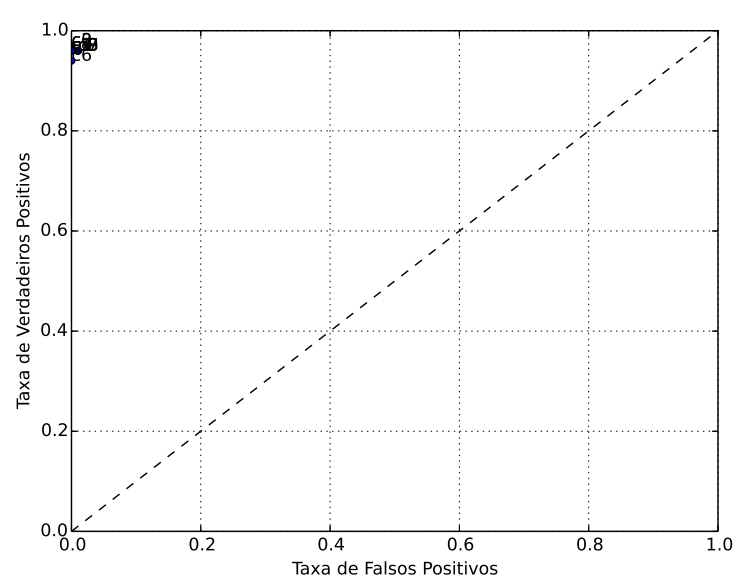

(b)

Figura 25 - (a)Modelos utilizando $C_{i j}=1.0$ para o conjunto de dados AID1608. (b)Modelos utilizando $C_{i j}=1.0$ para o conjunto de dados AID1608, com PU.

Cenário 3: Análise de classificadores ELM com fator de custo $C_{i j}=1.0+\frac{1}{\text { total }_{p}+\text { total }_{n}}$

Os resultados alcançados pelo classificador construído para os dados com rotulação original são apresentados na tabela 37. O melhor modelo apresentou acurácia igual a 100\%.

Tabela 52 - Resultados obtidos pelo classificador ELM com risco $C_{i j}=1.0+\frac{1}{\text { total }_{p}+\text { total }_{n}}$ para o conjunto de dados AID1608 antes da aplicação do rotulador PU.

\begin{tabular}{|c|c|c|c|c|c|c|c|c|c|c|c|c|c|c|}
\hline id & VP & FP & VN & FN & Pre. & Rec. & $t_{f p}$ & $t_{v p}$ & Acc & $f_{1}$ & Scale & Disc & Neu. & Fa. \\
\hline c0 & 65 & 1 & 964 & 3 & 0.98 & 0.96 & 0.0 & 0.96 & 1.0 & 0.97 & $\mathrm{~S}$ & EF & 1000 & sig \\
c1 & 66 & 3 & 962 & 2 & 0.96 & 0.97 & 0.0 & 0.97 & 1.0 & 0.96 & $\mathrm{~S}$ & EF & 1000 & rbf \\
c2 & 64 & 2 & 963 & 4 & 0.97 & 0.94 & 0.0 & 0.94 & 0.99 & 0.96 & $\mathrm{~S}$ & EF & 1500 & sig \\
c3 & 44 & 3 & 962 & 24 & 0.94 & 0.65 & 0.0 & 0.65 & 0.97 & 0.77 & $\mathrm{~S}$ & $\mathrm{EF}$ & 1500 & $\mathrm{rbf}$ \\
c4 & 44 & 2 & 963 & 24 & 0.96 & 0.65 & 0.0 & 0.65 & 0.97 & 0.77 & $\mathrm{~S}$ & $\mathrm{EF}$ & 500 & $\mathrm{sig}$ \\
c5 & 7 & 0 & 965 & 61 & 1.0 & 0.1 & 0.0 & 0.1 & 0.94 & 0.19 & - & - & 1500 & sig \\
c6 & 7 & 0 & 965 & 61 & 1.0 & 0.1 & 0.0 & 0.1 & 0.94 & 0.19 & - & - & 500 & sig \\
c7 & 7 & 2 & 963 & 61 & 0.78 & 0.1 & 0.0 & 0.1 & 0.94 & 0.18 & - & - & 1000 & sig \\
c8 & 33 & 323 & 642 & 35 & 0.09 & 0.49 & 0.33 & 0.49 & 0.65 & 0.16 & $\mathrm{~S}$ & $\mathrm{EF}$ & 500 & lin \\
c9 & 14 & 103 & 862 & 54 & 0.12 & 0.21 & 0.11 & 0.21 & 0.85 & 0.15 & - & - & 1500 & rbf \\
\hline
\end{tabular}


Para os experimentos após aplicação de PU, apesar da acurácia do modelo com melhor desempenho estar próxima de 100\%, a quantidade de neurônios na camada escondida foi de 1500, um número maior que outros classificadores semelhantes utilizando outros custos. Esse modelo também utilizou em sua fase de pré-processamento padronização e discretização EF.

Tabela 53 - Resultados obtidos pelo classificador ELM com risco $C_{i j}=1.0+\frac{1}{\text { total }_{p}+\text { total }_{n}}$ para o conjunto de dados AID1608 após a aplicação do rotulador PU.

\begin{tabular}{|c|c|c|c|c|c|c|c|c|c|c|c|c|c|c|}
\hline id & VP & FP & VN & FN & Pre. & Rec. & $t_{f p}$ & $t_{v p}$ & Acc & $f_{1}$ & Scale & Disc & Neu. & Fa. \\
\hline c0 & 68 & 1 & 964 & 0 & 0.99 & 1.0 & 0.0 & 1.0 & 1.0 & 0.99 & - & EW & 1500 & sig \\
c1 & 68 & 1 & 964 & 0 & 0.99 & 1.0 & 0.0 & 1.0 & 1.0 & 0.99 & - & EW & 1500 & sig \\
c2 & 66 & 6 & 959 & 2 & 0.92 & 0.97 & 0.01 & 0.97 & 0.99 & 0.94 & - & EF & 1500 & rbf \\
c3 & 66 & 6 & 959 & 2 & 0.92 & 0.97 & 0.01 & 0.97 & 0.99 & 0.94 & - & EF & 1500 & rbf \\
c4 & 62 & 4 & 961 & 6 & 0.94 & 0.91 & 0.0 & 0.91 & 0.99 & 0.93 & S & EF & 1000 & sig \\
c5 & 59 & 1 & 964 & 9 & 0.98 & 0.87 & 0.0 & 0.87 & 0.99 & 0.92 & S & EF & 1500 & sig \\
c6 & 57 & 2 & 963 & 11 & 0.97 & 0.84 & 0.0 & 0.84 & 0.99 & 0.9 & - & EF & 1000 & sig \\
c7 & 56 & 1 & 964 & 12 & 0.98 & 0.82 & 0.0 & 0.82 & 0.99 & 0.9 & - & EF & 1500 & sig \\
c8 & 57 & 2 & 963 & 11 & 0.97 & 0.84 & 0.0 & 0.84 & 0.99 & 0.9 & - & EF & 1000 & sig \\
c9 & 56 & 1 & 964 & 12 & 0.98 & 0.82 & 0.0 & 0.82 & 0.99 & 0.9 & - & EF & 1500 & sig \\
\hline
\end{tabular}

O desempenho dos melhores modelos também pode ser observado por meio da análise no espaço ROC, conforme a figura 26. Nota-se que, após a estimação dos rótulos via $\mathrm{PU}$, os pontos relativos aos classificadores avaliados moveram-se de áreas pouco favorecidas para áreas no espaço ROC associadas a melhores desempenhos. Avaliando o desempenho dos classificadores produzidos sob o espaço ROC, é possível observar que o melhor classificador, aqui identificado como c0, é posicionado em uma área do gráfico com baixa taxa de falsos positivos, e ao mesmo tempo, um alto número de verdadeiros positivos. Esta área é dita conservadora pois, o mesmo necessita de mais evidências para classificar uma instância como positiva, fato que justifica uma taxa de falso positivo baixa. 


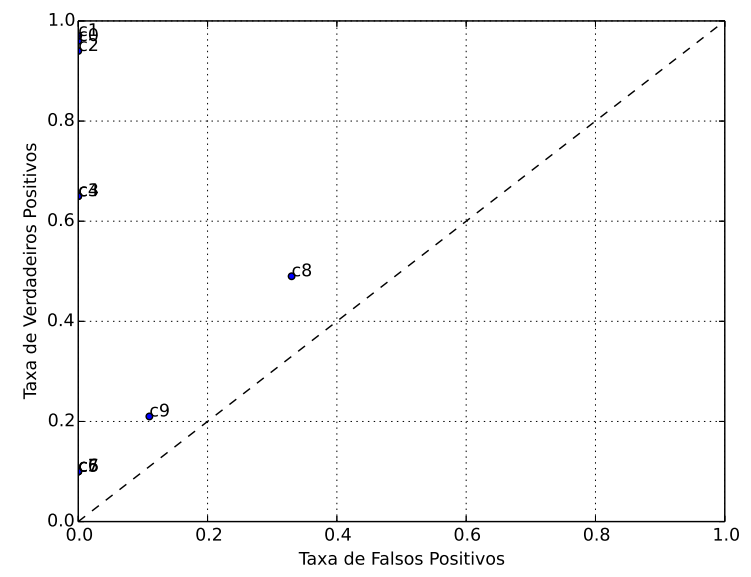

(a)

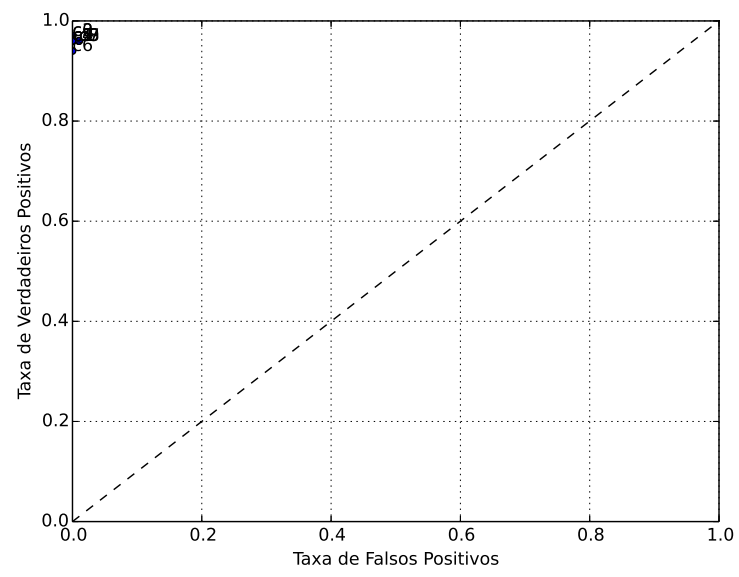

(b)

Figura 26 - (a)Modelos utilizando $C_{i j}=1.0+\frac{\text { total }_{p}}{\text { total }_{n}}$ para o conjunto de dados AID1608. (b)Modelos utilizando $C_{i j}=1.0+\frac{\text { total }_{n}}{\text { total }_{n}}$ para o conjunto de dados AID1608, com PU.

Em uma análise geral, para esse conjunto de dados, contendo erro majoritário de 93,41\%, foi possível observar a existência de várias configurações capazes de atingir acurácia próxima de $99 \%$, até mesmo com configurações de custo diferentes como por exemplo $C_{i j}=1.0+\frac{\text { total }_{p}}{\text { total }_{n}}$ e $C_{i j}=0.5+\frac{\text { total }_{p}}{\text { total }_{n}}$. Os melhores resultados utilizando estes dois custos concentram-se em modelos que utilizaram funções sigmoidais e com aplicação de discretização.

Os dados de QM utilizados no processo de desenvolvimento de novas drogas possuem características específicas que dificultam a aplicação de técnicas de aprendizagem de máquina em sua análise. Este trabalho destacou o tratamento de incerteza na definição do nível de atividade biológica dos compostos, por meio da aplicação da técnica PU para estimar uma rotulação confiável, pois a falta de dados confiáveis prejudica o desempenho de modelos de aprendizagem.

Na tabela 54 é possível observar, de forma geral, o desempenho obtido pelos métodos descritos neste projeto frente a trabalhos anteriores (SCHIERZ, 2009), que utilizaram os mesmos dados como estudo: 
Tabela 54 - Resultados de classificação obtidos neste trabalho em comparação com outros obtidos em trabalhos anteriores.

\begin{tabular}{|c|c|c|c|c|c|c|c|c|c|}
\hline & \multicolumn{3}{|c|}{ Trabalhos Anteriores } & \multicolumn{3}{c|}{ ELM } & \multicolumn{3}{|c|}{ ELM+PU } \\
\hline & TP\% & FP\% & Acc\% & Tp\% & FP\% & Acc\% & Tp\% & FP\% & Acc\% \\
\hline AID439 & 50.00 & 9.09 & 8.62 & 77.00 & 2.00 & 96.00 & 77.00 & 0.00 & 99.0 \\
AID644 & 53.85 & 14.29 & 75.61 & 99.00 & 33.00 & 77.00 & 99.00 & 0.00 & 99.0 \\
AID721 & 0.00 & 0.00 & 77.78 & 62.00 & 0.0 & 91.00 & 90.00 & 0.00 & 98.0 \\
AID1284 & 54.55 & 18.03 & 77.00 & 55.00 & 3.00 & 90.00 & 77.0 & 1.00 & 95.0 \\
AID1608 & 38.46 & 18.13 & NA & 96.00 & 0.01 & 98.9 & 100.0 & 0.00 & 99.9 \\
\hline
\end{tabular}

Em relação à tarefa de classificação executada pelo ELM, observa-se a obtenção de bons resultados, mesmo quando a quantidade de exemplos disoníveis para treinamento é consideravelmente menor que o universo de características disponíveis. Outra particularidade do modelo diz respeito a um parametro de regularização $\lambda$ utilizado no ajuste de estabilidade nos casos nos quais a matriz resultante das operações internas tende a singularidade.

\subsubsection{Análise da estimação correta dos rótulos}

O modelo proposto foi aplicado ao conjunto Iris bem como aos conjuntos de dados de QM citados anteriormente. Porém, uma comprovação efetiva da eficácia do modelo, seja na fase de estimação ou classificação, poderia ser feita pela realização de novos ensaios e testes biológicos. Considerando que essa abordagem está fora do contexto deste trabalho, uma proposta de validação é verificar a relação dos compostos alterados com o protocolo utilizado para a classificação dos mesmos durante a produção dos ensaios.

O conjunto de dados AID439 foi escolhido para esta verificação. Nesse ensaio 69 compostos que atingiram critérios específicos de seleção foram testados para determinar valores do índice de concentração efetiva ${ }^{3}\left(E C_{50}\right)$ individuais. Mais específicamente, esse índice representa a concentração do composto para qual 50\% do efeito é observado. Dessa forma, nesse ensaio, os compostos com valores de $E C_{50}$ maiores que $10 u M$ foram considerados inativos e os compostos com $E C_{50}$ iguais ou menores que $10 u M$ são considerados ativos.

3 Do original, em inglês, Half Effective Concentration. 
Na tabela 55, é possível observar os compostos que obtiveram rotulação alterada durante a execução do processo PU perto da região de limiar independente dos custos utilizados no processo de estimação de rótulos:

Tabela 55 - Tabela contendo os compostos que apresentaram alteração na rotulação em diversas configurações de peso, presentes no conjunto AID439.

\begin{tabular}{|c|c|c|}
\hline & Composto & Micromolar \\
\hline 661852 & $\mathrm{C}_{21} \mathrm{H}_{25} \mathrm{~N}_{5} \mathrm{O}_{2}$ & 14.7 \\
659072 & $\mathrm{C}_{26} \mathrm{H}_{25} \mathrm{~N}_{5} \mathrm{O}_{4}$ & 45.0 \\
657960 & $\mathrm{C}_{16} \mathrm{H}_{21} \mathrm{~N}_{3} \mathrm{OS}$ & 14.5 \\
647592 & $\mathrm{C}_{23} \mathrm{H}_{23} \mathrm{~N}_{5} \mathrm{O}$ & 45.0 \\
\hline
\end{tabular}

É possível observar que para os compostos 661852 e 667960 as modificações se restringem próximas à faixa onde se encontra o limiar de atividade definido neste ensaio, 10 $u M$. Em contra partida os compostos 659072 e 647592 apresentam um valor micromolar de 45. Neste último caso, por meio da análise do protocolo utilizado no experimento, notou-se que esses compostos foram submetidos a um processo diferente dos demais denominados de Ajuste de Curva, que estima um valor de $E C_{50}$, método este que é menos preciso do que aqueles executados manualmente.

Outro fator que deve ser avaliado, consiste na qualidade do conjunto de dados. Esta verificação consciste na aplicação de testes estatísticos com a finalidade de verificar a coesão do conjunto e a qualidade do ajuste feito na rotulação dos dados. Para efetuar tal procedimento é necessário considerar a dstribuição esperada em relação a distribuição obtida após o processo de rotulação.

O teste T-Student (SMITH, 2000), também conhecido como teste $t$, pode ser utilizado para comparar amostras com uma população, amostras pareadas ou até mesmo amostras independentes. Os valores obtidos durante este tipo de teste podem indicar que as amostras testadas não representam a população ou ainda ainda que pode ter ocorrido erro na obtenção dos dados.

Além de apresentar os valores críticos, a tabela a seguir exibe também os resultados obtidos com o teste T-Student, considerando os conjuntos de dados de QM, especificamente na fase de estimação dos rótulos, ou seja, durante o processo PU: 
Tabela 56 - Resultados obtidos após a aplicação do teste $t$ e valores críticos para análise bicaudal de $95 \%$

\begin{tabular}{|c|c|c|c|}
\hline Conjunto de Dados & Grau de Liberdade & Valor Crítico & Valor obtido \\
\hline AID1608 & 1031 & 1.282 & 0.786 \\
AID644 & 204 & 1.282 & 1,300 \\
AID1284 & 360 & 1.282 & 2.453 \\
AID721 & 92 & 1.289 & 1.579 \\
AID439 & 67 & 1.296 & 2.214 \\
\hline
\end{tabular}

Considerando os valores de referência existêntes na tabela de testes t-Student, e com uma área de avaliação tabulada de 0,025 na cauda superior, com valor base vairando entre 1.282 e 1.296. Dessa forma, é possível observar que, para o conjuntos de dados AID1608 os valores obtidos abaixo dos valores de refrência reforçam a aceitação do processo de classificação. Vale ressaltar também que esse conjunto, foi aquele que atingiu o resultado mais significativos também na etapa de estimação de rótulos. Outros conjuntos, com valores ligeiramente acima dos valores de referência, AID644 e AID721, também apresentaram resultados expressivos durante a execução do modelo proposto. Por outro lado, os modelos produzidos utilizando os conjuntos AID1284 e AID439 não se encontram no grupo dos classificadores que obtiveram os maióres índices de acurácia. Recomenda-se fortemente uma avaliação sobre o protocolo de obtenção de dados utilizados para estes conjuntos, bem como um número maior de exemplos para reavaliar o processo.

Dessa forma, é possível afirmar, mesmo que tecnicamente, que os elementos com rotulação alterada povoam a região de limite de atividade biológica. Entretanto, a atividade dos compostos, agora rotulados como positivos, só pode ser realmente confirmada após a aplicação de testes laboratoriais. 


\section{Conclusões}

A incerteza na rotulação de dados de QM interfere diretamente no funcionamento dos modelos de aprendizagem de máquina. Este problema, se não for tratado, prejudica o treinamento dos modelos, e consequentemente no processo de identificação de novas instâncias. Outro fator de impacto na análise de dados de QM diz respeito ao desbalanceamento, ou seja, uma maior incidência de elementos de uma determinada classe. Este cenário pode resultar em uma interpretação incorreta dos dados devido a viés causada pela classe com maior número de elementos no conjunto.

Para atenuar o impacto causado por essas particularidades foram adotadas uma série de medidas para cada problema separadamente. Devido à incerteza dos rótulos dos compostos, foram utilizadas técnicas de discretização não supervisionadas. Esse processo de discretização se faz necessário para definir intervalos para os atributos, abordagem esta que objetiva a melhora nos resultados dos modelos. Em especial nos momentos em que se combinava a técnica EW com a presença de dados contínuos, os resultados foram visivelmente insatisfatórios. Este fato pode estar associado à presença de diferentes escalas de um mesmo atributo, e a técnica EW, que calcula basicamente a fronteira dos intervalos, produziu grupos que contemplavam poucos objetos, prejudicando o desempenho final do modelo. Outra hipótese inicial foi de que, por meio da utilização dos dados binários e contínuos simultaneamente, os dados seriam mais representativos, fato este que não se confirmou conclusivamente após a análise dos resultados. Assim sugere-se fortemente que o uso de técnicas de seleção de características sejam utilizadas em trabalhos futuros.

Na etapa de estimação dos rótulos, a técnica de aprendizado semi-supervisionado PU foi aplicada com o objetivo de reduzir o efeito causado pela incerteza da rotulação, estimando novos rótulos para os compostos definidos como negativos ou com rotulação inconclusiva. Em um segundo momento, a rotulação estimada foi utilizada na construção de um classificador baseado em ELM.

As etapas de estimação e posterior classificação, foram adaptadas para uma abordagem de análise sensível ao custo, dessa forma, foi possível inserir um fator de ponderação para controlar o efeito causado pelo desbalanceamento entre as classes.

Por fim, apesar das críticas ao modelo por alguns pesquisadores, o classificador ELM se mostrou eficiente na resolução de diversos problemas relacionados a dados para 
aprendizagem de máquina, sendo que neste trabalho, o modelo obteve resultados significativos.

\subsection{Contribuições e Trabalhos Futuros}

As propostas de continuidade deste trabalho derivam das dificuldades encontradas no decorrer desta pesquisa, conforme descrito a seguir:

- Os conjuntos de dados envolvidos possuíam somente seus dados binários analisados em trabalhos anteriores. Neste trabalho, a inclusão dos dados contínuos simultaneamente não comprovou conclusivamente ganho de desempenho. Neste caso, uma proposta é o uso de métodos de seleção de características para melhorar os resultados obtidos na fase de estimação dos rótulos.

- Mesmo um especialista envolvido no processo de desenvolvimento de novos fármacos, possui dificuldades em definir o índice de atividade biológica para os compostos. Devido a essa indeterminação, além da estimação do rótulo, também poderá ser explorado a estimação do valor do índice de atividade biológica.

- Um dos fatores que contribuiram para os bons resultados, foi a inserção de um custo do erro de classificação nas técnicas PU e ELM. Contudo, uma técnica não baseada em força bruta pode ser útil para aproximar valores ótimos dessa ponderação.

- Com relação à incerteza dos rótulos, o aprendizado PU pode ser utilizado para encontrar uma área de indecisão e em seguida remover os exemplos que possuem baixos índices de confiança.

- Considera-se, por fim, interessante explorar a utilização do estimador PU na aplicação de técncias de reamostragem como seletor das instâncias que devem ser excluídas com a finalidade de balancear as classes envolvidas. 


\section{Referências $^{1}$}

BARATA, J. a. C. A.; HUSSEIN, M. S. The Moore-Penrose Pseudoinverse: A Tutorial Review of the Theory. Brazilian Journal of Physics, v. 42, n. 3, p. 146-165, 2012. ISSN 01039733. Citado na página 45.

BASKIN, I. I.; KIREEVA, N.; VARNEK, A. The one-class classification approach to data description and to models applicability domain. Molecular Informatics, v. 29, n. 8-9, p. 581-587, ago. 2010. ISSN 18681743. Disponível em: 〈http: //doi.wiley.com/10.1002/minf.201000063〉. Citado na página 27.

BATISTA, G. E. A. P. A.; PRATI, R. C.; MONARD, M. C. A Study of the Behavior of Several Methods for Balancing Machine Learning Training Data. SIGKDD Explor. Newsl., v. 6, n. 1, p. 20-29, 2004. ISSN 1931-0145. Disponível em:〈http://doi.acm.org/10.1145/1007730.1007735\$ \delimiter"026E30F\$nhttp: //dl.acm.org/ft $\{\backslash$ - gateway.cfm?id=1007735\{\&\}ty $\rangle$. Citado 2 vezes nas páginas 35 e 50.

BHARDWAJ, N.; GERSTEIN, M.; LU, H. Genome-wide sequence-based prediction of peripheral proteins using a novel semi-supervised learning technique. $B M C$ bioinformatics, v. 11 Suppl 1, p. S6, jan. 2010. ISSN 1471-2105. Disponível em: 〈http://www.pubmedcentral.nih.gov/articlerender.fcgi?artid=3009533\&tool= pmcentrez\&rendertype=abstract $\rangle. \quad$ Citado 2 vezes nas páginas 24 e 29.

BISHOP, C. M. Pattern Recognition and Machine Learning. [S.1.]: Springer, 2006. v. 4. 738 p. (Information science and statistics, 4). Citado 2 vezes nas páginas 21 e 42.

BLACKARD JOCK;J. DEAN, D. W. A. C. A. Covertype Data Set. 1998. Disponível em: 〈https://archive.ics.uci.edu/ml/datasets/Covertype〉. Citado na página 46.

BLANCHARD, G. Semi-Supervised Novelty Detection *. v. 11, p. 2973-3009, 2010. Citado na página 27.

CAMBRIA, E. et al. Extreme learning machines [trends controversies]. IEEE Intelligent Systems, v. 28, n. 6, p. 30-59, Nov 2013. ISSN 1541-1672. Citado na página 47.

DAUM, H.; MARCU, D. Domain Adaptation for Statistical Classifiers. v. 26, p. 101-126, 2006. Citado na página 23.

DEMPSTER a.P.; LAIRD, N.; RUBIN, D. Maximum likelihood from incomplete data via the EM algorithm. ournal of the Royal Statistical Society, Series B (Statistical Methodology), v. 39, n. 1, p. 1-38, 1977. Disponível em: 〈http://www.jstor.org/stable/10.2307/2984875〉. Citado na página 33.

DOMINGOS, P. MetaCost. Proceedings of the fifth ACM SIGKDD international conference on Knowledge discovery and data mining - KDD '99, p. 155-164, 1999. Disponível em: 〈http://portal.acm.org/citation.cfm?doid=312129.312220〉. Citado na página 37.

DOUGHERTY, J.; KOHAVI, R.; SAHAMI, M. Supervised and unsupervised discretization of continuous features. In: Machine Learning: Procedings of the Twelth International Conference. [S.1.]: Morgan Kaufmann, 1995. p. 194-202. Citado na página 55.

1 De acordo com a Associação Brasileira de Normas Técnicas. NBR 6023. 
DUDA, R. O.; HART, P. E.; STORK, D. G. Pattern Classification. [S.l.: s.n.], 2000. 680 p. Citado na página 56.

DUDA, R. O. et al. Pattern Classification, 2nd Ed. 2001. Citado na página 48.

EGAN, J. P. Signal Detection Theory and ROC Analysis. [S.1.]: Academic Press, 1975. (Series in Cognition and Perception). Citado na página 51.

FAWCETT, T. An introduction to ROC analysis. Irbm, v. 35, n. 6, p. 299-309, 2014. ISSN 18760988. Citado 4 vezes nas páginas 49, 50, 51 e 52.

FERREIRA, S. P. Análise de Dados Ecológicos. Epilepsy behavior EB, v. 13, p. 316-22, 2004. Disponível em: $\langle$ http://scholar.google.com/scholar?hl=en\&btnG=Search\&q=intitle: ?+An?lise+de+Dados+Ecol?gicos $\rangle$. Citado na página 28.

FERRI, C.; HERNÁNDEZ-ORALLO, J.; MODROIU, R. An experimental comparison of performance measures for classification. Pattern Recognition Letters, v. 30, n. 1, p. 27-38, 2009. ISSN 01678655. Citado na página 49.

FONSECA, N. A.; COSTA, V. S. k-RNN : k-Relational Nearest Neighbour Algorithm. 2008. Citado na página 29.

GERTRUDES, J. C. Redução de dimensionalidade e expansão de conjunto dados na química medicinal. 2012. Citado na página 22.

HAJIAN-TILAKI, K. Receiver Operating Characteristic (ROC) Curve Analysis for Medical Diagnostic Test Evaluation. Caspian J Intern Med, v. 4, n. 2, p. 627-635, 2013. ISSN 2008-6164. Disponível em: 〈http://www.ncbi.nlm.nih.gov/pubmed/24009950〉. Citado na página 51.

HARRISON, D.; RUBINFELD, D. Hedonic prices and the demand for clean air. 1978. 〈https://archive.ics.uci.edu/ml/datasets/Housing $\rangle$. Citado na página 46.

HUANG, G. et al. Video analysis using spatiotemporal descriptor and kernel extreme learning machine for lip reading. Neural Networks, Electron. Imaging, 2015. Disponível em:〈http://electronicimaging.spiedigitallibrary.org/article.aspx?〉 Citado na página 43.

HUANG, G.-B. What are extreme learning machines? filling the gap between frank rosenblatt's dream and john von neumann's puzzle. 2015. Citado na página 47.

HUANG, G.-B.; ZHU, Q.-Y.; SIEW, C.-K. Extreme learning machine: Theory and applications. Neurocomputing, v. 70, n. 1-3, p. 489-501, dez. 2006. ISSN 09252312. Disponível em: 〈http://linkinghub.elsevier.com/retrieve/pii/S0925231206000385〉. Citado 2 vezes nas páginas 42 e 45.

JOIţA, D. Unsupervised static discretization methods in data mining. 2010. Citado na página 55 .

KARPOV, P. V. et al. Virtual screening based on one-class classification. Doklady Chemistry, v. 437, n. 2, p. 107-111, maio 2011. ISSN 0012-5008. Disponível em: 〈http://link.springer.com/10.1134/S0012500811040082〉. Citado na página 27.

KATSIKIS, V. N.; PAPPAS, D. Fast computing of the Moore-Penrose inverse matrix. Electronic Journal of Linear Algebra, v. 17, n. November, p. 637-650, 2008. ISSN 10813810. Citado na página 44. 
KONG, X.; NG, M. K.; ZHOU, Z.-h. Transductive Multilabel Learning via Label Set Propagation. v. 25, n. 3, p. 704-719, 2013. Citado na página 35.

KRAWCZYK, B.; WO, M. Combining Diverse One-Class Classifiers. p. 590-601, 2012. Citado na página 27.

LECUN'S, Y. What's so great about Extreme Learning Machines? 2015. Citado na página 48.

LICHMAN, M. UCI Machine Learning Repository. 2013. Disponível em: 〈http: //archive.ics.uci.edu/ml $\rangle$. Citado na página 56.

LING, C. X.; SHENG, V. S. Cost-sensitive learning and the class imbalance problem. Encyclopedia of Machine Learning, p. 231-235, 2008. Disponível em: 〈http: //www.springer.com/computer/ai/book/978-0-387-30768-8\$ delimiter"026E30F \$nhttp: //citeseerx.ist.psu.edu/viewdoc/download?doi=10.1.1.164.4418 $\{\&\} \mathrm{rep}=\operatorname{rep} 1\{\&\}$ ty $\rangle$. Citado 3 vezes nas páginas 35, 36 e 37.

LIU, B. et al. Partially Supervised Classification of Text Documents. ICML 2002 Proceedings of the Nineteenth International Conference on Machine Learning, p. 387-394, 2002. Citado na página 38.

LIU, N.; WANG, H. Ensemble based extreme learning machine. IEEE Signal Processing Letters, v. 17, n. 8, p. 754-757, Aug 2010. ISSN 1070-9908. Citado 2 vezes nas páginas 46 e 47.

LUSCI, A.; POLLASTRI, G.; BALDI, P. Deep architectures and deep learning in chemoinformatics: the prediction of aqueous solubility for drug-like molecules. Journal of chemical information and modeling, v. 53, n. 7, p. 1563-75, jul. 2013. ISSN 1549-960X. Disponível em: 〈http://www.pubmedcentral.nih.gov/articlerender.fcgi?artid=3739985\& tool $=$ pmcentrez\&rendertype $=$ abstract $\rangle. \quad$ Citado na página 28.

M. Bazaraa, W. Nonlinear programming - theory and algorithms 3rd ed - m. bazaraa, et al., (wiley, 2006) ww (1).pdf. 2006. Citado na página 43.

MINSKY MARVIN;PAPERT, S. Progress report on artificial intelligence. 1971. Citado na página 47.

MITCHELL, T. M. Generative and discriminative Classifiers: Naive Bayes and Logistic Regression. Access, p. 1-17, 1997. Citado na página 33.

MITCHELL, T. M. Machine Learning. [S.l.]: McGraw-Hill Science, 1997. v. 17. Citado 3 vezes nas páginas 21, 32 e 42.

NASRI, N. et al. Chemical compounds from Phoenician juniper berries (Juniperus phoenicea). Natural product research, v. 2338, n. 94, p. 715-721, 2011. ISSN 1478-6427. Disponível em: 〈http://www.ncbi.nlm.nih.gov/pubmed/21707254〉. Citado na página 28.

PEREIRA, F. et al. BMC bioinformatics, v. 77, 2013. ISSN 16603397. Citado na página 35.

PETKOVI, M. D. Iterative method for computing Moore-Penrose inverse based on Penrose equations. n. 2, p. 1604-1613, 2011. Citado na página 44. 
PROVOST, F.; FAWCETT, T.; KOHAVI, R. The Case Against Accuracy Estimation for Comparing Induction Algorithms. Proceedings of the Fifteenth International Conference on Machine Learning, p. 445-453, 1997. Citado na página 51.

PUBCHEM. National Center for Biotechnology Information.PubChem BioAssay Database. 2016. 〈https://pubchem.ncbi.nlm.nih.gov/bioassay/1608〉. [Online; accessed 18-out-2016]. Citado na página 62.

PUBCHEM. National Center for Biotechnology Information.PubChem BioAssay Database. 2016. 〈https://pubchem.ncbi.nlm.nih.gov/bioassay/644〉. [Online; accessed 18-out-2016]. Citado na página 63.

PUBCHEM. National Center for Biotechnology Information.PubChem BioAssay Database. 2016. 〈https://pubchem.ncbi.nlm.nih.gov/bioassay/644〉. [Online; accessed 18-out-2016]. Citado na página 63.

PUBCHEM. National Center for Biotechnology Information.PubChem BioAssay Database. 2016. 〈https://pubchem.ncbi.nlm.nih.gov/bioassay/721〉. [Online; accessed 18-out-2016]. Citado na página 63.

PUBCHEM. National Center for Biotechnology Information.PubChem BioAssay Database. 2016. 〈https://pubchem.ncbi.nlm.nih.gov/bioassay/439〉. [Online; accessed 18-out-2016]. Citado na página 63.

RALAIVOLA, L. et al. Graph kernels for chemical informatics. Neural networks : the official journal of the International Neural Network Society, v. 18, n. 8, p. 1093-110, out. 2005. ISSN 0893-6080. Disponível em:〈http://www.ncbi.nlm.nih.gov/pubmed/16157471〉. Citado 2 vezes nas páginas 28 e 29.

SCHIERZ, A. C. Virtual screening of bioassay data. Journal of Cheminformatics, v. 1, n. 1, p. 1-12, 2009. ISSN 17582946. Citado 2 vezes nas páginas 36 e 105.

SEO, Y.-w.; SYCARA, K. Cost-Sensitive Access Control for Illegitimate. Access, p. 117-128, 2006. Citado na página 36.

SHENG, V.; LING, C. Thresholding for making classifiers cost-sensitive. Proceedings of the National Conference on Artificial Intelligence, p. 476-481, 2006. Disponível em: 〈http://www.aaai.org/Papers/AAAI/2006/AAAI06-076.pdf〉. Citado 2 vezes nas páginas 35 e 36 .

SMITH, B. C. et al. SIRT3 substrate specificity determined by peptide arrays and machine learning. ACS chemical biology, v. 6, n. 2, p. 146-57, fev. 2011. ISSN 1554-8937. Disponível em: 〈http://www.pubmedcentral.nih.gov/articlerender.fcgi?artid $=3042044 \&$ tool $=$ pmcentrez\&rendertype=abstract $\rangle$. Citado na página 45.

SMITH, P. P. F. Types of Data, Desciptive Statistics, and Statistical Test for Nominal Data. 2000. Citado na página 107.

SOKOLOVA, M.; LAPALME, G. A systematic analysis of performance measures for classification tasks. Information Processing and Management, v. 45, n. 4, p. 427-437, 2009. ISSN 03064573. Citado na página 51. 
TERMENON, M. et al. Extreme learning machines for feature selection and classification of cocaine dependent patients on structural MRI data. Neural Processing Letters, v. 38, p. 375-387, 2013. ISSN 13704621. Citado na página 43.

VARNEK, A.; BASKIN, I. Machine learning methods for property prediction in chemoinformatics: Quo Vadis? Journal of chemical information and modeling, v. 52, n. 6, p. 1413-37, jun. 2012. Citado 2 vezes nas páginas 22 e 24.

VISHWANATHAN, S. V. N. et al. Graph kernels. Journal of Machine Learning Research, v. 11, p. 1201-1242, 2010. ISSN 1793-5091. Citado na página 28.

WEISS, G. M.; PROVOST, F. Learning when training data are costly: The effect of class distribution on tree induction. Journal of Artificial Intelligence Research, v. 19, p. 315-354, 2003. ISSN 10769757. Citado na página 35.

WIESLER, S.; RICHARD, A.; SCHL, R. Mean-Normalized Stochastic Gradient for Large-Scale Deep Learning. v. 1, n. 2, p. 180-184, 2014. Citado na página 54.

YOUNG, D. C. Computacional Drug Design. [S.l.]: John Wiley \& Sons, Inc, 2009. Citado 2 vezes nas páginas 21 e 29.

ZADROZNY, B.; LANGFORD, J.; ABE, N. Cost-Sensitive Learning by CostProportionate Example Weighting. ICDM '03 Proceedings of the Third IEEE International Conference on Data Mining, p. 435, 2003. ISSN 15504786. Citado 3 vezes nas páginas 24, 36 e 37.

ZWEIG, M. H.; CAMPBELL, G. Receiver-oprating characteristics (ROC) plots - a fundamental evaluation tool in clinical medicine. Clinical Chemistry, v. 39, n. 4, p. 561-577, 1993. ISSN 0009-9147. Citado na página 51. 